\title{
Synthesis and aggregation studies of functional binaphthyl- bridged chiral phthalocyanines
}

\author{
Miguel Á. Revuelta-Maza, ${ }^{a}$ Tomás Torres ${ }^{* a, b, c}$ and Gema de la Torre*a,b
}

a. Universidad Autónoma de Madrid, c/ Francisco Tomás y Valiente 7, 28049 Madrid, Spain. Email:miguel.revuelta@uam.es, tomas.torres@uam.es; gema.delatorre@uam.es

b. Institute for Advanced Research in Chemical Sciences (IAdChem), Universidad Autónoma de Madrid, 28049 Madrid, Spain.

c. Instituto Madrileño de Estudios Avanzados (IMDEA)-Nanociencia, c/ Faraday 9, Cantoblanco, 28049 Madrid, Spain.

\section{EXPERIMENTAL}

\section{General:}

Chemicals were purchased from commercial suppliers and used without further purification unless stated otherwise. Compounds $\mathbf{7 a},{ }^{1,2} \mathbf{7} \mathbf{b},{ }^{3,4} \mathbf{8} \mathbf{a},{ }^{5} \mathbf{8} \mathbf{b},{ }^{6} \mathbf{8} \mathbf{d},{ }^{7} \mathbf{9},{ }^{8}$ and $\mathbf{1 0}^{9}$ have been prepared according to published procedures. The monitoring of the reactions has been carried out by thin layer chromatography (TLC), employing aluminum sheets coated with silica gel type 60 F254 (0.2 $\mathrm{mm}$ thick, E. Merck). Purification and separation of the synthesized products was performed by column chromatography, using silica gel (230-400 mesh, 0.040-0.063 mm, Merck). Eluents and relative proportions of the solvents are indicated for each particular case. Size exclusion chromatography was performed using Bio-Beads S-X1 (200-400 mesh, Bio-Rad). Infrared (IR) spectra were recorded on an Agilent Technologies Cary 630 FTIR spectrophotometer, employing in all cases solid samples (diamond ATR). Mass Spectrometry (MS) and High Resolution Mass Spectrometry (HRMS) spectra were recorded employing Fast Atom Bombardment (FAB-MS), $\mathrm{APCl}$ or Matrix Assisted Laser Desorption/Ionization-Time of Flight (MALDI-TOF), using a Waters VG-AutoSpec spectrometer for FAB-MS, a Bruker Ultraflex III TOF/TOF spectrometer, with a NdYAG laser operating at $335 \mathrm{~nm}$, for MALDI-TOF, and a Bruker MAXIS II, with Q-TOF analyzer, for $\mathrm{APCl}$. The different matrixes employed are indicated for each spectrum. Mass spectrometry data are expressed in $\mathrm{m} / \mathrm{z}$ units. All MS experiments were carried out at the Servicio Interdepartmental de Investigación (SIdl) of the Universidad Autónoma de Madrid. NMR spectra $\left({ }^{1} \mathrm{H}-\mathrm{NMR},{ }^{13} \mathrm{C}-\mathrm{NMR}\right)$ were recorded on a Bruker AC-300 (300 MHz) instrument or a Bruker XRD$500(500 \mathrm{MHz}) . \mathrm{H}$ assignation for ${ }^{1} \mathrm{H}-\mathrm{NMR}$ are indicated in the Supporting Information. UV-Vis and fluorescence spectra were recorded with a JASCO V-660 and a JACSO FP-8600 spectrophotometer, respectively, using spectroscopic grade solvents. The logarithm of the molar absorption coefficient $\varepsilon$ is expressed in between parentheses. UV-Vis, fluorescence and CD spectroscopy measurements were performed using quartz cuvettes $(1 \mathrm{~cm})$. Circular 
dichroism (CD) spectra were recorded on a Jasco J-815 CD-spectrometer including a Jasco Peltier ETCT-762 temperature controller.

\section{Synthesis of bisphthalonitriles $7 c$ and $7 d$}

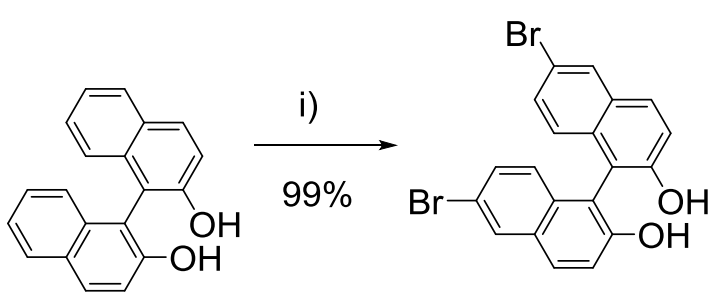

(R)-9

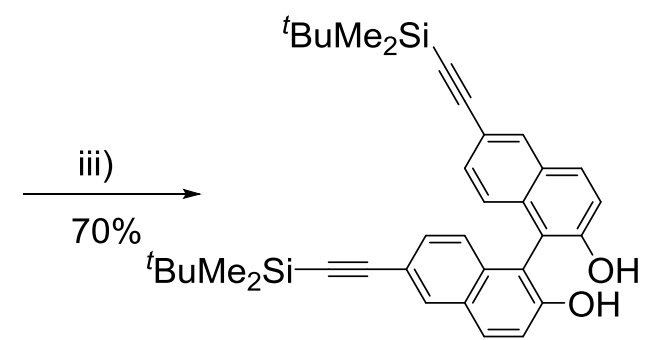

(R)-10

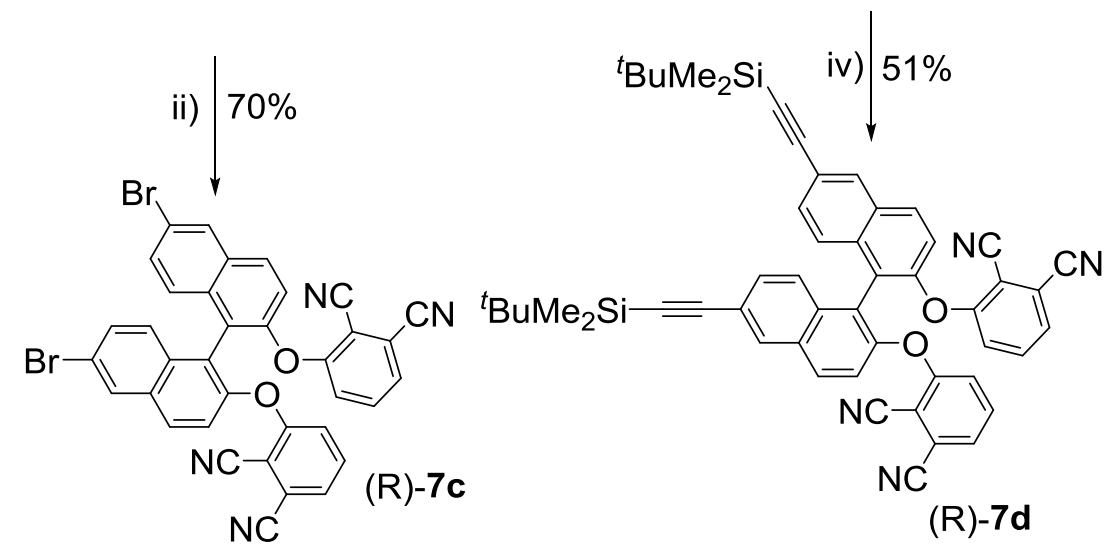

Scheme S1. i) $\mathrm{Br}_{2}, \mathrm{DCM},-78^{\circ} \mathrm{C}$ to r.t., 3.5h; ii) 3-nitrophthalonitrile, $\mathrm{K}_{2} \mathrm{CO}_{3}$, DMSO, r.t., 5h; iii) ${ }^{t} \mathrm{BuMe}_{2} \mathrm{Si}$-acetylene, $\mathrm{PdCl}_{2}\left(\mathrm{PPh}_{3}\right)_{2}, \mathrm{Cul}, \mathrm{Et}_{3} \mathrm{~N}, 60^{\circ} \mathrm{C}$, 4 h; iv) 3-nitrophthalonitrile, $\mathrm{K}_{2} \mathrm{CO}_{3}$, DMSO, r.t., 48h.

Synthesis of 7c: To a solution of 3-nitrophthalonitrile $(0.858 \mathrm{~g}, 4.95 \mathrm{mmol})$ and (R)- or (S)-6,6'dibromo-BINOL $9(1 \mathrm{~g}, 2.25 \mathrm{mmol})$ in dry DMSO $(10 \mathrm{~mL})$ at room temperature, potassium carbonate $(1.15 \mathrm{~g}, 8.33 \mathrm{mmol})$ was added in several portions. The mixture was stirred at room temperature under an argon atmosphere for $5 \mathrm{~h}$. The reaction mixture was poured into ice water $(100 \mathrm{~mL})$, filtered and the solid was washed with water $(250 \mathrm{~mL})$ and dried. The product was recrystallized in EtOAc, giving the product as a yellowish solid. Yield: $1.10 \mathrm{~g}(70 \%) .{ }^{1} \mathrm{H}-\mathrm{NMR}(300$ $\left.\mathrm{MHz}, \mathrm{DMSO}-\mathrm{d}_{6}\right): \delta 7.18\left(\mathrm{~d}, J=9.04 \mathrm{~Hz}, 2 \mathrm{H}, \mathrm{HAr}-\mathrm{b}_{\mathrm{i}}\right), 7.30\left(\mathrm{~d}, J=8.50 \mathrm{~Hz}, 2 \mathrm{H}, \operatorname{HAr}-\mathrm{a}_{\mathrm{i}}\right), 7.48(\mathrm{~d}, J=$ $\left.9.04 \mathrm{~Hz}, 2 \mathrm{H}, \mathrm{HAr}-\mathrm{b}_{\mathrm{i}}\right), 7.62\left(\mathrm{~m}, 4 \mathrm{H}, \mathrm{HAr}-\mathrm{a}_{\mathrm{i}}\right), 7.67\left(\mathrm{~m}, 2 \mathrm{H}, \mathrm{HAr}-\mathrm{d}_{\mathrm{i}}\right), 8.22\left(\mathrm{~d}, J=8.90 \mathrm{~Hz}, 2 \mathrm{H}, \mathrm{HAr}-\mathrm{d}_{\mathrm{i}}\right)$, 8.39 (s, 2H, HAr-c). ${ }^{13} \mathrm{C}-\mathrm{NMR}(75 \mathrm{MHz}$, DMSO-d 6 ): $\delta 104.6,112.6,115.3,115.6,119.4,121.0$, 121.6, 121.9, 127.4, 128.2, 130.3, 130.5, 130.8, 131.5, 132.3, 135.7, 150.0, 158.8. HR-MS for $\mathrm{C}_{36} \mathrm{H}_{16} \mathrm{Br}_{2} \mathrm{~N}_{4} \mathrm{O}_{2}$ : APCl Positive (ULTRAFLEX II), apci + fia hplc1100 $\mathrm{t}=300 . \mathrm{m}$; $\mathrm{MeOH}$ as ionizing phase; $[\mathrm{M}+\mathrm{H}]^{+} 726.9975$ (calculated), 726.9995 (experimental); $[\mathrm{M}+\mathrm{H}+\mathrm{MeOH}]^{+}$is also observed. IR(ATR) v $v^{-1}\left(\mathrm{~cm}^{-1}\right): 3074,2227$ (CEN st), 1568, 1491, 1449, 1350, 1319, 1258, 801. $[\alpha]_{D}^{20}=+71.6^{\circ}$ (c $=0.4, \mathrm{CH}_{2} \mathrm{Cl}_{2}$ ) for $(\mathrm{R})-8 \mathrm{c}$; and $[\alpha]_{D}^{20}=-78.7^{\circ}\left(\mathrm{c}=0.4, \mathrm{CH}_{2} \mathrm{Cl}_{2}\right.$ ) for (S)-8c. m.p. $=280-282^{\circ} \mathrm{C}$.

Synthesis of 7d: To a solution of 3-nitrophthalonitrile (154 mg, $0.888 \mathrm{mmol})$ and (R)-BINOL derivative $10(200 \mathrm{mg}, 0.355 \mathrm{mmol})$ in dry DMSO $(5 \mathrm{~mL})$ at room temperature, potassium carbonate $(245 \mathrm{mg}, 1.78 \mathrm{mmol}$ ) was added in several portions. The mixture was stirred at room temperature under an argon atmosphere for $48 \mathrm{~h}$. The reaction mixture was poured into ice 
water $(100 \mathrm{~mL})$, and the aqueous layer was extracted with ethyl acetate. After being dried with anhydrous $\mathrm{MgSO}_{4}$, the organic layer was evaporated, and the residue was purified by silica gel chromatography (heptane:EtOAc, 3:1), giving the product as a withe solid. Yield: $147 \mathrm{mg}(51 \%)$. ${ }^{1} \mathrm{H}-\mathrm{NMR}\left(300 \mathrm{MHz}, \mathrm{CDCl}_{3}\right.$ ): $\delta 0.14(\mathrm{~s}, 12 \mathrm{H}, \mathrm{Me}-\mathrm{d}), 0.94\left(\mathrm{~s}, 18 \mathrm{H},{ }^{\mathrm{t}} \mathrm{Bu}-\mathrm{e}\right), 7.12(\mathrm{~d}, J=8.75 \mathrm{~Hz}, 2 \mathrm{H}$, HAr- $\left.b_{i}\right), 7.19-7.28\left(m, 8 H, H A r-a_{i}, b_{i}, d_{i}\right), 7.48\left(t, J=8.32 \mathrm{~Hz}, 2 \mathrm{H}, \operatorname{HAr}-a_{i}\right), 7.91(\mathrm{~d}, J=9.01 \mathrm{~Hz}, 2 \mathrm{H}$, HAr- $\left.\mathrm{d}_{\mathrm{i}}\right), 8.01$ (s, 2H, HAr-c). ${ }^{13} \mathrm{C}-\mathrm{NMR}\left(75 \mathrm{MHz}, \mathrm{CDCl}_{3}\right): \delta-4.5,16.9,26.3,77.2,105.2,105.9,109.1$, $112.3,115.0,117.1,120.0,121.4,121.6,122.6,125.5,127.5,130.6,131.1,131.4,132.5,133.0$, 134.9, 150.3, 159.8. HR-MS for $\mathrm{C}_{52} \mathrm{H}_{46} \mathrm{~N}_{4} \mathrm{O}_{2} \mathrm{Si}_{2}$ : $\mathrm{FAB}$ El+, Matrix: m-NBA, HR FB+; $[\mathrm{M}+\mathrm{H}]^{+}$ 815,32375 (calculated), 815.3237 (experimental); $\left[\mathrm{M}+\mathrm{H}^{-}{ }^{+} \mathrm{Bu}\right]^{+}$is also observed. IR(ATR) $\mathrm{v}^{-1}\left(\mathrm{~cm}^{-}\right.$ $\left.{ }^{1}\right): 3073,2951,2928,2855,2232$ (C=N st), 2150 (C=C st), 1574, 1458, 1347, 1268, 1208, 823. $[\alpha]_{D}^{20}=+63.8^{\circ}\left(\mathrm{c}=0.3, \mathrm{CH}_{2} \mathrm{Cl}_{2}\right)$ for (R)-7d. m.p. $=278-280^{\circ} \mathrm{C}$.

\section{Synthesis of phthalocyanines:}

\section{General procedure for the synthesis of ZnPcs 1-6:}

Bishthalonitrile AA 7 (1 eq.), phthalonitrile $B 8$ (2 eq.) and anhydrous $\mathrm{Zn}(\mathrm{AcO})_{2}(1.5-2$ eq.) were placed in a $5 \mathrm{~mL}$ high pressure resistant flask equipped with a magnetic stirrer, and then dry $O$ dichlorobenzene/DMF (dried over 4Å molecular sieves) 2:1 were added, for [AA 7] $=0.05 \mathrm{M}$. The mixture was heated to $150-160^{\circ} \mathrm{C}$ overnight under argon atmosphere. After cooling, the solvent was removed under vacuum. The mixture of products was then purified as follows.

Synthesis of ZnPc 1: Following the general procedure: 7a $(63.6 \mathrm{mg}, 0.118 \mathrm{mmol}), 8 \mathrm{a}(60 \mathrm{mg}$, $0.236 \mathrm{mmol})$ and $\mathrm{Zn}(\mathrm{OAc})_{2}(43.3 \mathrm{mg}, 0.236 \mathrm{mmol})$. Solid was initially washed with heptane and purified by column chromatography on $\mathrm{SiO}_{2}$ (THF/heptane from 1:2 to 1:1). The first compound to elute was $\mathbf{B}_{4}-\mathbf{a}$, unequivocally confirmed by MALDI-TOF mass spectrometry, sequentially followed by the target AABB 1 and $\mathbf{A}_{4}$-a which show similar $R_{f}$. To achieve full separation after common silica gel column chromatography, an additional column chromatography on Bio-Beads using THF as eluent was necessary. Elution sequence was monitored by semipreparative chiral HPLC (see Figure below). Unfortunately HPLC separation is not good enough, preventing the semipreparative application of this technique. After evaporation of the solvent, a blue solid was obtained, which was washed with MeOH. Yield: $14.1 \mathrm{mg}$, (11\%). ${ }^{1} \mathrm{H}-\mathrm{NMR}$ (500 MHz, DMSO-d 6 ): 6 7.50, 7.62, 7.84, 7.94, 7.97, 8.18, 8.28 ( $\mathrm{m}, 12 \mathrm{H}, \mathrm{HAr}-\mathrm{b}, \mathrm{c})$; 8.35, 8.43, 8.66, 8.77, 8.91, 8.97, 8.99, 9.13, 9.29, $9.45\left(\mathrm{~m}, 12 \mathrm{H}, \mathrm{HAr}-\mathrm{a}_{\mathrm{i}}\right)$. HR-MS for $\mathrm{C}_{52} \mathrm{H}_{24} \mathrm{I}_{2} \mathrm{~N}_{8} \mathrm{O}_{2} \mathrm{Zn}$ : MALDI (ULTRAFLEX III); DCTB + PPGNa 1000; 1109.9398 (calculated), 1109.9385 (experimental). IR(ATR) $v^{-1}\left(\mathrm{~cm}^{-1}\right): 2960,2923$, 2853, 1260, 1089, 1021, 804. UV-Vis (THF) $\log \left(\varepsilon / \mathrm{M}^{-1} \mathrm{~cm}^{-1}\right)(\lambda): 4.8(343 \mathrm{~nm}) ; 4.5(612 \mathrm{~nm}) ; 5.3$ $(678 \mathrm{~nm})$. Fluorescence: $\lambda_{\text {maximum }}=690 \mathrm{~nm} ; \lambda_{\text {excitation }}=668 \mathrm{~nm}$. 


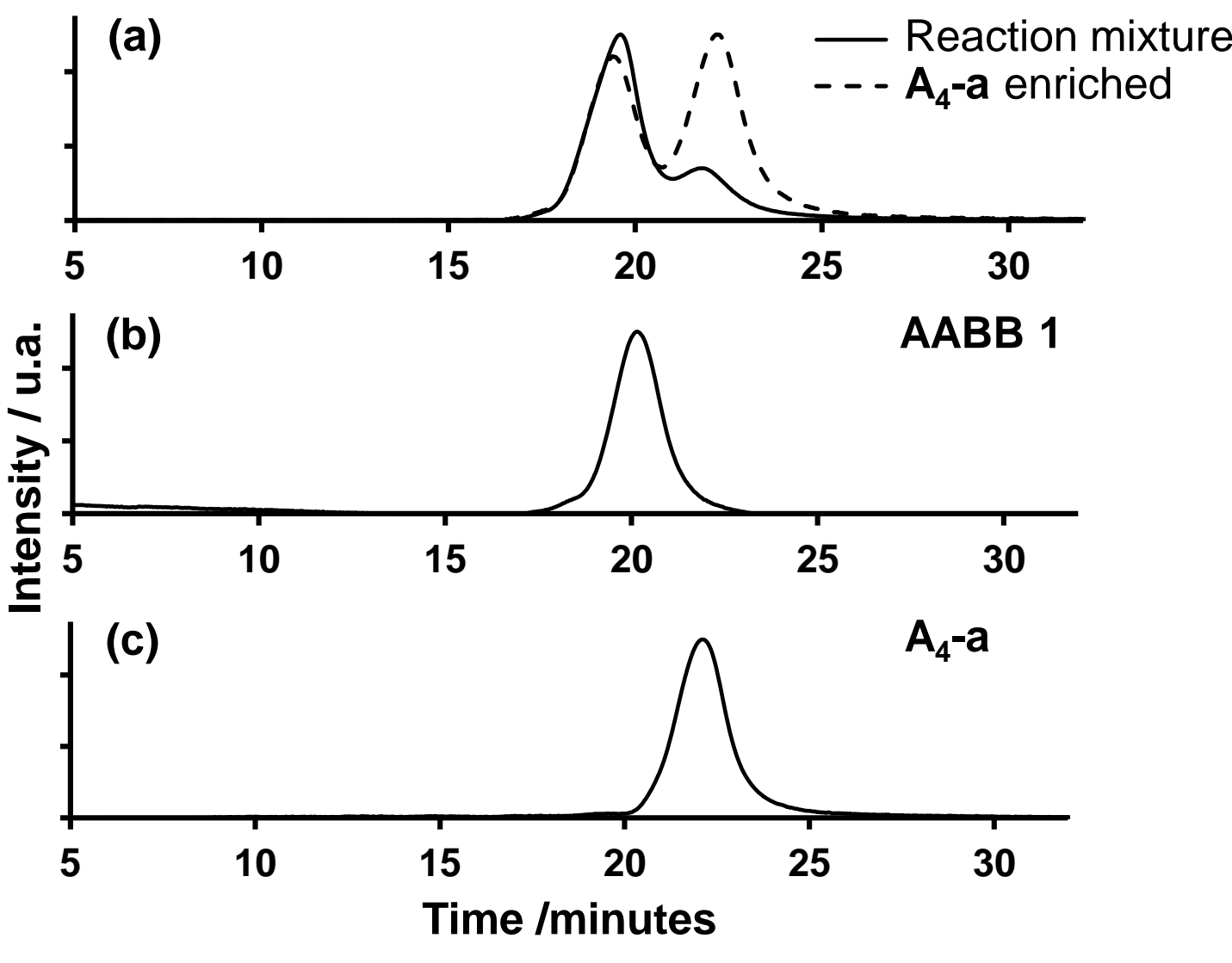

Figure SE. HPLC chromatograms of: (a) AABB $1+\mathbf{A}_{4}$-1a reaction mixture (continuous line) and reaction mixture enriched with $\mathbf{A}_{\mathbf{4}}-\mathbf{1 a}$ (dashed line); (b) pure AABB $\mathbf{1}$; and (c) pure $\mathbf{A}_{\mathbf{4}}-\mathbf{1} \mathbf{a}$. Semipreparative chiral column chromatography for HPLC: Chiralpack IC $10 \mathrm{~mm} \phi \times 250 \mathrm{~mL}$, particle size $5 \mu \mathrm{m}$, Part No. 83335, Daicel Corporation. Conditions: flow 1mL/min, chloroform:THF (99:1), $\mathrm{T}=25^{\circ} \mathrm{C}$, detection by UV-Vis at $350 \mathrm{~nm}$ and $676 \mathrm{~nm}$; injection volume $15 \mu \mathrm{L}$ from a solution of the AABB 1 and $(\mathbf{A A})_{2}$-a mixture $\sim 1 \mathrm{mg} / \mathrm{mL}$.

Synthesis of ZnPc 2: Following the general procedure: 7b (60 mg, $0.079 \mathrm{mmol}), 8 a$ ( $40 \mathrm{mg}, 0.157$ $\mathrm{mmol}$ ) and $\mathrm{Zn}(\mathrm{OAc})_{2}(21.6 \mathrm{mg}, 0.1180 \mathrm{mmol})$. Solid was initially washed with heptane and purified by column chromatography on $\mathrm{SiO}_{2}$ (dioxane/heptane, 1:4). Symmetrical phthalocyanine $(\mathbf{A A})_{\mathbf{2}}$-b was eluted in the first fraction, followed by $\mathrm{ZnPc} \mathbf{2}$, and finally $\mathbf{B}_{\mathbf{4}}$-a. The fraction containing ZnPc $\mathbf{2}$ was further purified by an additional column chromatography on BioBeads using THF as eluent. After evaporation of the solvent, a blue solid was obtained, which was washed with MeOH. Yield: $35 \mathrm{mg},(33 \%) .{ }^{1} \mathrm{H}-\mathrm{NMR}$ (500 MHz, THF- $\left.\mathrm{d}_{8}, 363 \mathrm{~K}\right): \delta 0.84(\mathrm{~m}, 6 \mathrm{H}$, $\left.\mathrm{CH}_{3}-\mathrm{d}_{8}\right) ; 1.29\left(\mathrm{~m}, 12 \mathrm{H}, \mathrm{CH}_{2}-\mathrm{d}_{5-7}\right) ; 1.40\left(\mathrm{~m}, 4 \mathrm{H}, \mathrm{CH}_{2}-\mathrm{d}_{4}\right) ; 1.47\left(\mathrm{~m}, 4 \mathrm{H}, \mathrm{CH}_{2}-\mathrm{d}_{3}\right) ; 1.83\left(\mathrm{~m}, 4 \mathrm{H}, \mathrm{CH}_{2}-\mathrm{d}_{2}\right)$; $2.88\left(\mathrm{~m}, 4 \mathrm{H}, \mathrm{CH}_{2}-\mathrm{d}_{1}\right) ; 7.46,7.54,7.61,7.71,7.80,7.90,7.99,8.01,8.06$ (m, 16H, HAr-ai, $\left.\mathrm{b}_{\mathrm{i}}, \mathrm{c}\right) ; 8.25$, 8.39, 8.52, 8.69, 8.82, 8.91, 9.00, $9.11\left(\mathrm{~m}, 6 \mathrm{H}, \mathrm{HAr}-\mathrm{a}_{\mathrm{i}}\right)$. HR-MS for $\mathrm{C}_{68} \mathrm{H}_{56} \mathrm{I}_{2} \mathrm{~N}_{8} \mathrm{O}_{2} \mathrm{Zn}$ : MALDI (ULTRAFLEX III); DCTB + PEGNa 1500; 1334.1902 (calculated), 1334.1877 (experimental). IR(ATR) $\mathrm{V}^{-1}\left(\mathrm{~cm}^{-1}\right): 2961,2922,2853,1259,1088,1017,798$. UV-Vis (THF) $\log \left(\varepsilon / \mathrm{M}^{-1} \mathrm{~cm}^{-1}\right)(\lambda): 4.7(343$ $\mathrm{nm}) ; 4.5(612 \mathrm{~nm}) ; 5.2(678 \mathrm{~nm})$. Fluorescence: $\lambda_{\text {maximum }}=690 \mathrm{~nm} ; \lambda_{\text {excitation }}=668 \mathrm{~nm}$.

Synthesis of ZnPc 3: Following the general procedure: $7 \mathbf{b}$ ( $52 \mathrm{mg}, 0.068 \mathrm{mmol}), \mathbf{8 b}$ ( $52 \mathrm{mg}, 0.136$ $\mathrm{mmol})$ and $\mathrm{Zn}(\mathrm{OAc})_{2}(19 \mathrm{mg}, 0.102 \mathrm{mmol})$. Solid was initially washed with heptane and purified 
by column chromatography on $\mathrm{SiO}_{2}$ (dioxane/heptane, 1:2). (AA) $)_{2}$-b was eluted in the first fraction, followed by ZnPc 3, and finally $\mathbf{B}_{4}-\mathbf{b}$. The fraction containing $\mathrm{ZnPc} \mathbf{3}$ was further purified by an additional column chromatography on Bio-Beads using THF as eluent. After evaporation of the solvent, a blue solid was obtained, which was washed with $\mathrm{MeOH}$. Yield: $18 \mathrm{mg}$, (17\%). ${ }^{1} \mathrm{H}-\mathrm{NMR}\left(500 \mathrm{MHz}, \mathrm{THF}-\mathrm{d}_{8}, 363 \mathrm{~K}\right): \delta 0.83\left(\mathrm{~m}, 6 \mathrm{H}, \mathrm{CH}_{3}-\mathrm{d}_{8}\right) ; 1.28\left(\mathrm{~m}, 12 \mathrm{H}, \mathrm{CH}_{2}-\mathrm{d}_{5,6,7}\right) ; 1.41(\mathrm{~m}, 4 \mathrm{H}$, $\left.\mathrm{CH}_{2}-\mathrm{d}_{4}\right) ; 1.47\left(\mathrm{~m}, 4 \mathrm{H}, \mathrm{CH}_{2}-\mathrm{d}_{3}\right) ; 1.82\left(\mathrm{~m}, 4 \mathrm{H}, \mathrm{CH}_{2}-\mathrm{d}_{2}\right) ; 2.87\left(\mathrm{~m}, 4 \mathrm{H}, \mathrm{CH}_{2}-\mathrm{d}_{1}\right) ; 7.33,7.50,7.69,7.80$, 7.95, $8.15\left(\mathrm{~m}, 16 \mathrm{H}, \mathrm{HAr}-\mathrm{a}_{\mathrm{i}}, \mathrm{b}_{\mathrm{i}}, \mathrm{c}\right)$; $9.14,9.36\left(\mathrm{~m}, 4 \mathrm{H}, \mathrm{HAr}-\mathrm{a}_{\mathrm{i}}\right)$. HR-MS for $\mathrm{C}_{68} \mathrm{H}_{54} \mathrm{l}_{4} \mathrm{~N}_{8} \mathrm{O}_{2} \mathrm{Zn}$ : MALDI (ULTRAFLEX III); DCTB + PPGNa 1000 + PPGNa 2000; 1585.9834 (calculated), 1585.9808 (experimental). IR(ATR) $v^{-1}\left(\mathrm{~cm}^{-1}\right):$ 2951, 2919, 2850, 1578, 1475, 1386, 1332, 1259, 1087, 1019, 800. UV-Vis (THF) $\log \left(\varepsilon / \mathrm{M}^{-1} \mathrm{~cm}^{-1}\right)(\lambda): 4.8(357 \mathrm{~nm}) ; 4.5(618 \mathrm{~nm}) ; 5.2(685 \mathrm{~nm})$. Fluorescence: $\lambda_{\text {maximum }}=694 \mathrm{~nm} ; \lambda_{\text {excitation }}=680 \mathrm{~nm}$.

Synthesis of ZnPc 4: Following the general procedure: 7c (100 mg, $0.144 \mathrm{mmol}), 8 \mathrm{c}(36.8 \mathrm{mg}$, $0.287 \mathrm{mmol})$ and $\mathrm{Zn}(\mathrm{OAc})_{2}(39.5 \mathrm{mg}, 0.215 \mathrm{mmol})$. Solid was initially washed with $\mathrm{MeOH}$ and filtered in a silica gel plug with THF. After evaporation of the solvent, the product was purified by column chromatography on Bio-Beads using THF as eluent, monitored by TLC (heptane:THF, $3: 1,1 \%$ pyridine), being the second eluted fraction. After evaporation of the solvent, a blue solid was obtained, which was washed with MeOH. Yield: $30 \mathrm{mg}$, (21\%). ${ }^{1} \mathbf{H}-\mathbf{N M R}$ (500 MHz, DMSO$\left.d_{6}, 363 \mathrm{~K}\right): \delta 7.65$ (d, J = $\left.9.96 \mathrm{~Hz}, 2 \mathrm{H}, \mathrm{HAr}-\mathrm{a}_{\mathrm{i}}\right) ; 7.71\left(\mathrm{~d}, J=9.41 \mathrm{~Hz}, 2 \mathrm{H}, \mathrm{HAr}-\mathrm{b}_{\mathrm{i}}\right) ; 7.75$ (d, J = $9.41 \mathrm{~Hz}$, $\left.2 \mathrm{H}, \mathrm{HAr}-\mathrm{b}_{\mathrm{i}}\right) ; 7.98\left(\mathrm{~d}, J=9.68 \mathrm{~Hz}, 2 \mathrm{H}, \mathrm{HAr}-\mathrm{b}_{\mathrm{i}}\right) ; 8.04\left(\mathrm{~d}, J=9.68 \mathrm{~Hz}, 2 \mathrm{H}, \mathrm{HAr}-\mathrm{b}_{\mathrm{i}}\right) ; 8.14(\mathrm{~m}, 4 \mathrm{H}, \mathrm{HAr}-$ $\left.a_{i}\right) ; 8.24\left(m, 2 H, H A r-a_{i}\right) ; 8.25$ (s, 2H, HAr-c); 9.19 (m, 2H, HAr-ai ); 9.26 (m, 4H, HAr-e). HR-MS for $\mathrm{C}_{52} \mathrm{H}_{24} \mathrm{Br}_{2} \mathrm{~N}_{8} \mathrm{O}_{2} \mathrm{Zn}$ : MALDI (ULTRAFLEX III); DCTB + PPGNa 1000; 1013.9675 (calculated); 1013.9681 (experimental). IR(ATR) $v^{-1}\left(\mathrm{~cm}^{-1}\right): 2917,2850,1575,1484,1332,1270,1260,1121$, 1090. UV-Vis (THF) $\log \left(\varepsilon / \mathrm{M}^{-1} \mathrm{~cm}^{-1}\right)(\lambda): 4.6(343 \mathrm{~nm}) ; 4.3(607 \mathrm{~nm}) ; 5.1(673 \mathrm{~nm})$. Fluorescence: $\lambda_{\text {maximum }}=684 \mathrm{~nm} ; \lambda_{\text {excitation }}=663 \mathrm{~nm}$.

Synthesis of ZnPc 5: Following the general procedure: 7c (100 mg, $0.144 \mathrm{mmol}), 8 d$ (102 mg, $0.287 \mathrm{mmol})$ and $\mathrm{Zn}(\mathrm{OAc})_{2}(39.5 \mathrm{mg}, 0.215 \mathrm{mmol})$. The mixture was purified by column chromatography on $\mathrm{SiO}_{2}$ (THF/heptane 2:1) containing the first fraction a mixture of $\mathbf{B}_{4}-\mathbf{d}$ and $\mathrm{ZnPc} 5$, and the second fraction $(\mathbf{A A})_{2}-\mathrm{c}$. The first fraction was purified by column chromatography on $\mathrm{SiO}_{2}$ (THF/heptane 1:1 with pyridine $1 \%$ ) being the first fraction $\mathbf{B}_{4}-\mathbf{d}(10 \mathrm{mg}$, yield: $9 \%$ ) and the second fraction the desired ZnPc 5. After evaporation of the solvent, a blue solid was obtained, which was washed with $\mathrm{MeOH}$. Yield: $12 \mathrm{mg},(6 \%) .{ }^{1} \mathrm{H}-\mathrm{NMR}(500 \mathrm{MHz}$, DMSO-d $\left.\mathrm{d}_{6}, 363 \mathrm{~K}\right): \delta 1.09\left(\mathrm{~m}, 12 \mathrm{H}, \mathrm{CH}_{3}-\mathrm{d}_{5}\right) ; 1.60\left(\mathrm{~m}, 8 \mathrm{H}, \mathrm{CH}_{2}-\mathrm{d}_{4}\right) ; 1.71\left(\mathrm{~m}, 8 \mathrm{H}, \mathrm{CH}_{2}-\mathrm{d}_{3}\right) ; 2.10(\mathrm{~m}$, $\left.8 \mathrm{H}, \mathrm{CH}_{2}-\mathrm{d}_{2}\right) ; 4.70\left(\mathrm{~m}, 8 \mathrm{H}, \mathrm{CH}_{2}-\mathrm{d}_{1}\right) ; 7.73\left(\mathrm{~m}, 2 \mathrm{H}, \mathrm{HAr}-\mathrm{a}_{\mathrm{i}}\right) ; 7.84\left(\mathrm{~m}, 6 \mathrm{H}, \mathrm{HAr}-\mathrm{b}_{\mathrm{i}}\right) ; 8.23\left(\mathrm{~m}, 2 \mathrm{H}, \mathrm{HAr}-\mathrm{a}_{\mathrm{i}}\right)$; $8.39\left(\mathrm{~m}, 2 \mathrm{H}, \mathrm{HAr}-\mathrm{b}_{\mathrm{i}}\right)$; 8.91 (m, 4H, HAr-ai, c); 9.16 (m, 2H, HAr-ai ); 9.40 (m, 2H, HAr-ai ). HR-MS for $\mathrm{C}_{76} \mathrm{H}_{64} \mathrm{Br}_{2} \mathrm{~N}_{8} \mathrm{O}_{10} \mathrm{Zn}$ : MALDI (ULTRAFLEX III); DCTB + PEGNa 1500; 1470.2398 (calculated), 1470.2386 (experimental). IR(ATR) $\mathrm{V}^{-1}\left(\mathrm{~cm}^{-1}\right)$ : 2956, 1724, 1577, 1485, 1322, 1269, 1090. UV-Vis $(\mathrm{THF}) \log \left(\varepsilon / \mathrm{M}^{-1} \mathrm{~cm}^{-1}\right)(\lambda): 4.8(346 \mathrm{~nm}) ; 4.5(614 \mathrm{~nm}) ; 5.3(681 \mathrm{~nm})$. Fluorescence: $\lambda_{\text {maximum }}=698$ $\mathrm{nm} ; \lambda_{\text {excitation }}=670 \mathrm{~nm}$.

Synthesis of (AA) $)_{2}-\mathrm{c}$ : This product was obtained in the synthesis of $\mathrm{ZnPc} 5$ as secondary product in the second fraction of the first column chromatography on silica gel. After evaporation of the solvent, the product was purified by column chromatography on Bio-Beads using toluene as eluent. After evaporation of the solvent, a blue solid was obtained, which was washed with MeOH. Yield: 14 mg, (13\%). ${ }^{1} \mathrm{H}-\mathrm{NMR}$ (500 MHz, DMSO-d $\left.\mathrm{d}_{6}, 363 \mathrm{~K}\right)$ : $\delta 7.57$ (m, 4H, HAr-a); 7.68, 7.60 (m, 8H; HAr-bi ); 7.98, 8.01 (m, 8H, HAr-bi ); 8.22 (m, 8H, HAr-a,c); 9.27 (m, 4H,HAr-a). HRMS for $\mathrm{C}_{72} \mathrm{H}_{32} \mathrm{Br}_{4} \mathrm{~N}_{8} \mathrm{O}_{4} \mathrm{Zn}$ : MALDI (ULTRAFLEX III); DCTB + PPGNa 1000 + PPGNa 2000; 1451.8566 (calculated); 1451.8511 (experimental). IR(ATR) $\mathrm{v}^{-1}\left(\mathrm{~cm}^{-1}\right):$ 1577, 1483, 1330, 1267, 1260, 1198, 
1103. UV-Vis (THF) $\log \left(\varepsilon / \mathrm{M}^{-1} \mathrm{~cm}^{-1}\right)(\lambda): 4.5(345 \mathrm{~nm}) ; 4.2(613 \mathrm{~nm}) ; 5.0(684 \mathrm{~nm})$. Fluorescence: $\lambda_{\text {maximum }}=693 \mathrm{~nm} ; \lambda_{\text {excitation }}=674 \mathrm{~nm}$.

Synthesis of ZnPc 6: Following the general procedure: 7d (45 mg, $0.055 \mathrm{mmol}$ ), 8 a ( $28 \mathrm{mg}, 0.110$ $\mathrm{mmol}$ ) and $\mathrm{Zn}(\mathrm{OAc})_{2}(11.1 \mathrm{mg}, 0.061 \mathrm{mmol})$. Solid was purified by column chromatography on $\mathrm{SiO}_{2}$ (THF/heptane from 1:4 to 1:2). The first eluted fraction contains a mixture of (AA) 2 -d and $\mathrm{ZnPc}$ 6, followed by a second fraction with $\mathbf{B}_{\mathbf{4}}-\mathbf{a}$. First fraction was further purified by two additional column chromatography on Bio-Beads using THF as eluent. First eluted fraction contains (AA) ${ }_{2}-d$ (traces), and AABB 6 is in the second fraction. After evaporation of the solvent, a blue solid was obtained, which was washed with MeOH. Yield: $10.3 \mathrm{mg},(13 \%) .{ }^{1} \mathrm{H}-\mathrm{NMR}$ (500 MHz, THF- $\left.\mathrm{d}_{8}, 323 \mathrm{~K}\right): \delta 0.22$ (m, 12H, Me-d); 1.05 (m, 18H, $\left.{ }^{t} \mathrm{Bu}-\mathrm{e}\right) ; 7.67\left(\mathrm{~m}, 2 \mathrm{H}, \mathrm{HAr}-\mathrm{b}_{\mathrm{i}}\right) ; 7.77-7.85$ $\left(\mathrm{m}, 4 \mathrm{H}, \mathrm{HAr}-\mathrm{a}_{i}, \mathrm{~b}_{\mathrm{i}}\right)$; 7.95-8.02 (m, 4H, HAr- $\left.\mathrm{b}_{\mathrm{i}}\right)$; 8.08-8.30 (m, 6H, HAr- $\left.\mathrm{a}_{i}, \mathrm{c}\right)$; 8.45-8.53 (m, 2H, HAr$\left.\mathrm{a}_{\mathrm{i}}\right) ; 8.65,7.72,8.78\left(\mathrm{~m}, 2 \mathrm{H}, \mathrm{HAr}-\mathrm{a}_{\mathrm{i}}\right) ; 8.95,9.06,9.15\left(\mathrm{~m}, 2 \mathrm{H}, \mathrm{HAr}-\mathrm{a}_{\mathrm{i}}\right)$. HR-MS for $\mathrm{C}_{68} \mathrm{H}_{52} \mathrm{I}_{2} \mathrm{~N}_{8} \mathrm{O}_{2} \mathrm{Si}_{2} \mathrm{Zn}$ : MALDI (ULTRAFLEX III); DCTB + PEGNa 1500; 1386.1127 (calculated), 1386.1101 (experimental). IR(ATR) $v^{-1}\left(\mathrm{~cm}^{-1}\right):$ :2923, 2853, 2147, 1730, 1576, 1470, 1337, 1254, 1088, 827. UV-Vis (THF) $\log \left(\varepsilon / \mathrm{M}^{-1} \mathrm{~cm}^{-1}\right)(\lambda): 4.6(350 \mathrm{~nm}) ; 4.4(611 \mathrm{~nm}) ; 5.2(678 \mathrm{~nm})$. Fluorescence: $\lambda_{\text {maximum }}=688 \mathrm{~nm}$; $\lambda_{\text {excitation }}=668 \mathrm{~nm}$.

Synthesis of (AA) $)_{2}$-d: As $(A A)_{2}$-d was obtained in the synthesis of ZnPc 6 with very low yield, it was prepared independently by mixing $7 \mathbf{d}(75 \mathrm{mg}, 0.092 \mathrm{mmol})$ and $\mathrm{Zn}(\mathrm{OAC})_{2}(13 \mathrm{mg}, 0.069$ $\mathrm{mmol}$ ) in dry o-dichlorobencene:DMF (2:1), for $[\mathbf{8 d}]=0.05 \mathrm{M}$ at $160^{\circ} \mathrm{C}$ overnight. Solid was purified by column chromatography on $\mathrm{SiO}_{2}$ (dioxane/heptane, 1:1). The mixture was further purified by two additional column chromatography on Bio-Beads using first toluene and then THF as eluents. After evaporation of the solvent, a blue solid was obtained, which was washed with MeOH. Yield: $5.2 \mathrm{mg}$, (7\%). ${ }^{1} \mathrm{H}-\mathrm{NMR}\left(500 \mathrm{MHz}, \mathrm{THF}-\mathrm{d}_{8}, 298 \mathrm{~K}\right): \delta 0.19$ (m, 24H, Me-d); 1.01

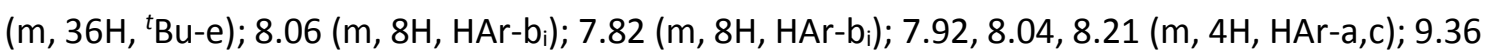
(m, 4H, HAr-a). HR-MS for $\mathrm{C}_{104} \mathrm{H}_{92} \mathrm{~N}_{8} \mathrm{O}_{4} \mathrm{Si}_{4} \mathrm{Zn}$ : MALDI (ULTRAFLEX III); DCTB + PEGNa 1500; 1692.5605 (calculated), 1692.5578 (experimental). IR(ATR) $v^{-1}\left(\mathrm{~cm}^{-1}\right): 2953,2147,1710,1574$, 1470, 1358, 1250, 1219, 1087, 825. UV-Vis (THF) $\log \left(\varepsilon / \mathrm{M}^{-1} \mathrm{~cm}^{-1}\right)(\lambda): 4.7(299 \mathrm{~nm}) ; 4.7(349 \mathrm{~nm})$; $4.4(614 \mathrm{~nm}) ; 5.2(684 \mathrm{~nm})$. Fluorescence: $\lambda_{\text {maximum }}=694 \mathrm{~nm} ; \lambda_{\text {excitation }}=680 \mathrm{~nm}$.

\section{REFERENCES}

[1] C. C. Leznoff, S. M. Marcuccio, S. Greenberg, A. B. P. Lever, K. B. Tomer, Can. J. Chem. $1985,63,623-631$.

[2] N. Kobayashi, Chem. Commun. 1998, 2, 487-488.

[3] W. Lv, X. Zhang, J. Lu, Y. Zhang, X. Li, J. Jiang, Eur. J. Inorg. Chem. 2008, 27, 4255-4261.

[4] Q.-S. Hu, X.-F. Zheng, L. Pu, J. Org. Chem. 1996, 61, 5200-5201.

[5] S. M.Marcuccio, P. I. Svirskaya, S. Greenberg, A. B. P. Lever, C. C. Leznoff, K. B. Tomer, Can. J. Chem. 1985, 63, 3057-3069.

[6] D. S. Terekhov, K. J. M. Nolan, C. R. McArthur, C. C. Leznoff, J. Org. Chem. 1996, 61, 30343040 . 
[7] B. Tylleman, R. Gómez-Aspe, G. Gbabode, Y. H. Geerts and S. Sergeyev, Tetrahedron, $2008,64,4155$.

[8] G. D. Y. Sogah, D. J. Cram, J. Am. Chem. Soc. 1979, 101, 3035-3042.

[9] H. Sasai, T. Tokunaga, S. Watanabe, T. Suzuki, N. Itoh, M. Shibasaki, J. Org. Chem. 1995, $60,7388-7389$. 


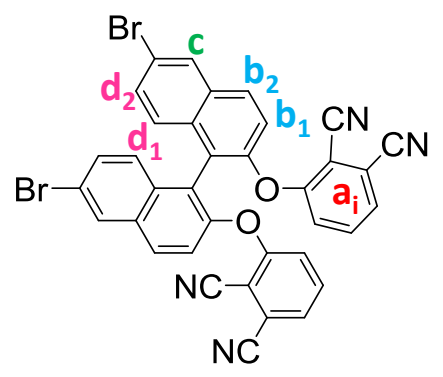

Figure S1. Structure of $7 c$ and $H$ assignation for ${ }^{1} H-N M R$.

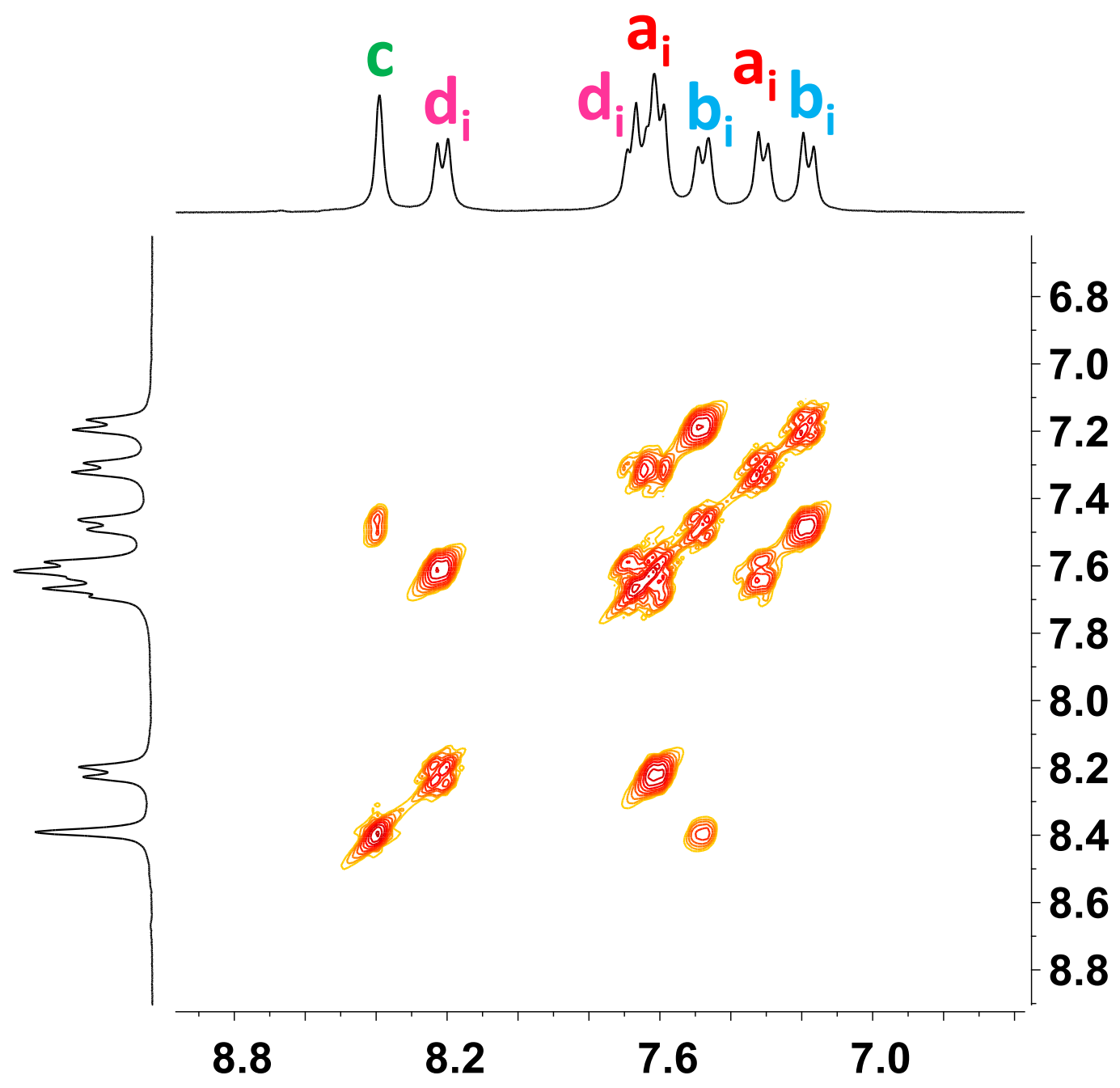




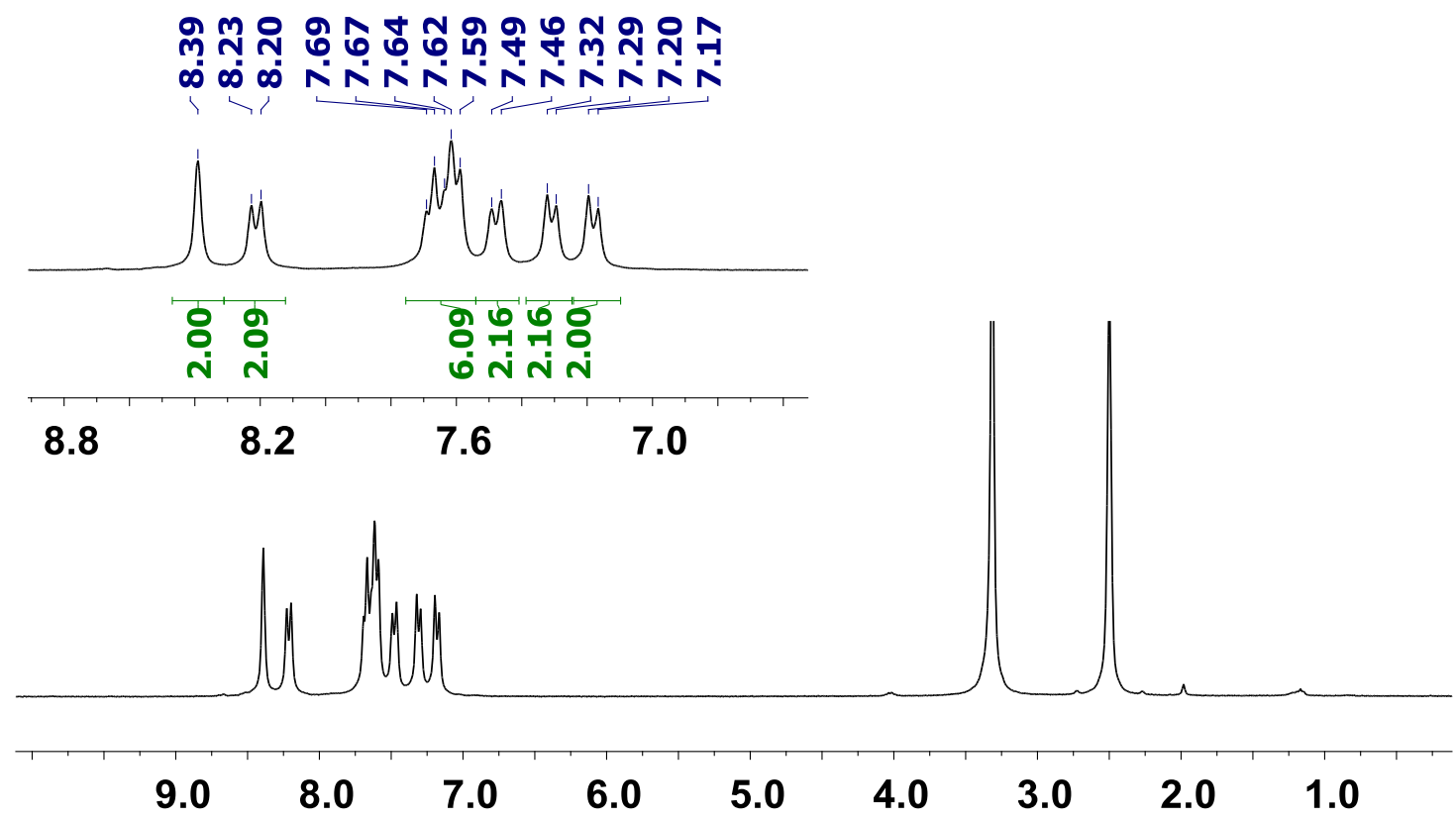

Figure S2. ${ }^{1} \mathrm{H}-\mathrm{NMR}$ and COSY of $7 \mathrm{c}$ in DMSO- $\mathrm{d}_{6}$, at $323 \mathrm{~K}$.
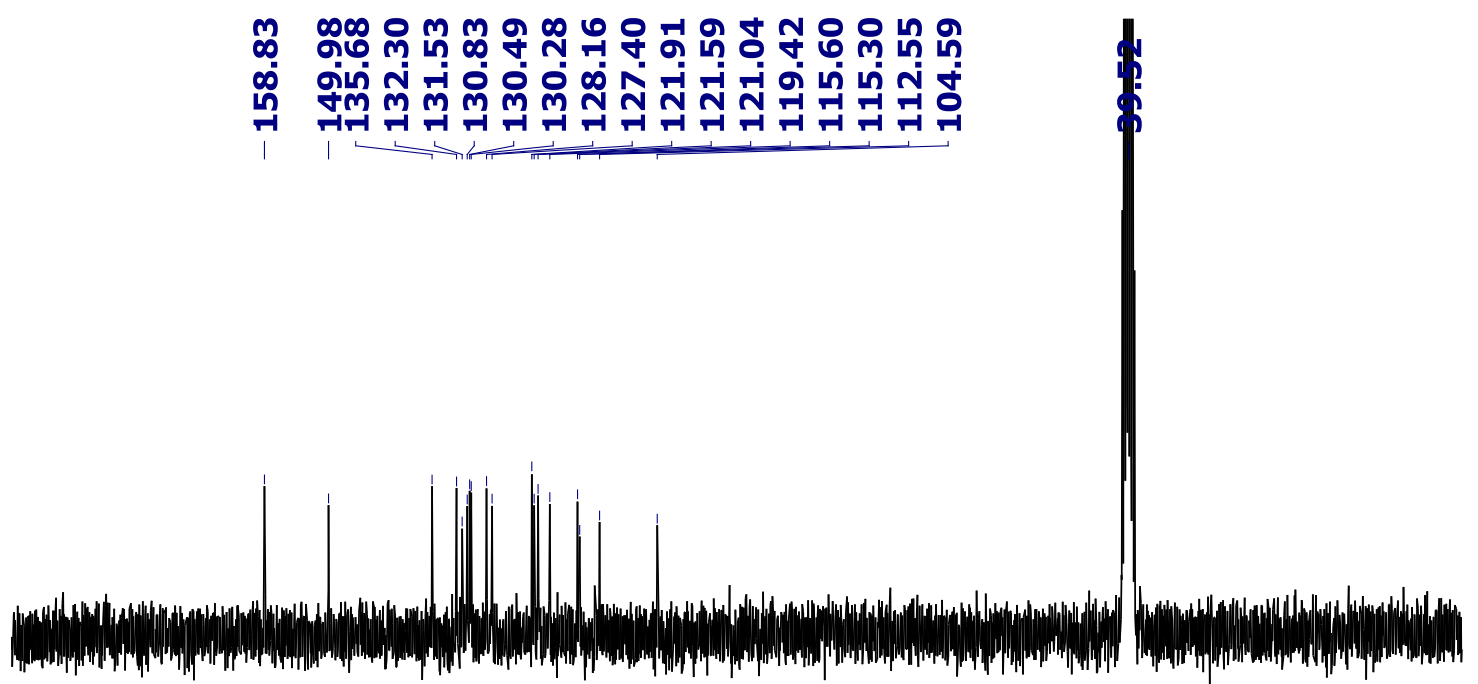

170

140

110

80

60

40

20

Figure S3. ${ }^{13} \mathrm{C}-\mathrm{NMR}$ of $7 \mathrm{c}$ in DMSO- $\mathrm{d}_{6}$. 

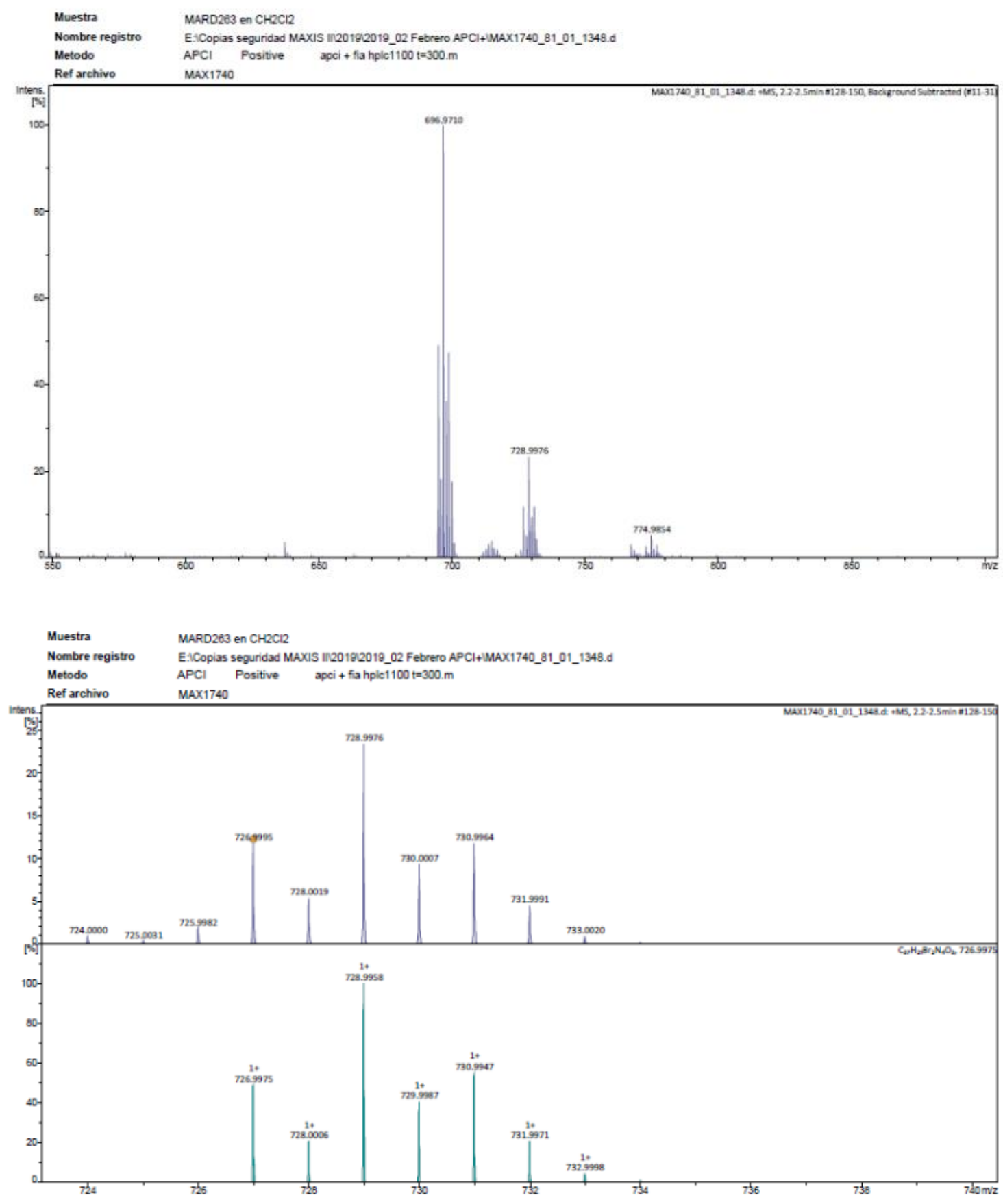

Figure S4. MS and HR-MS of 7c. 


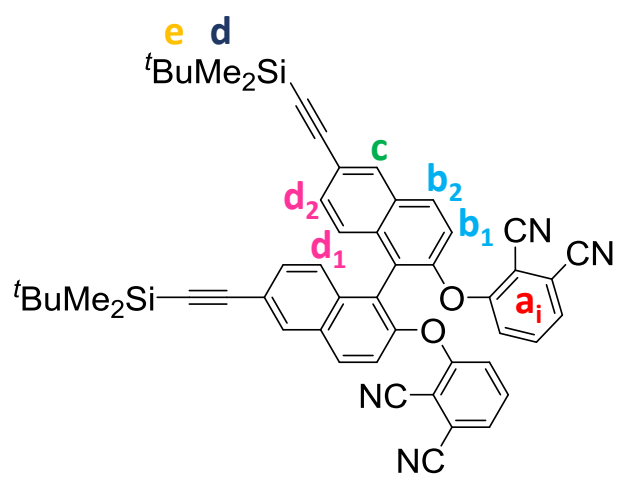

Figure S5. Structure of $\mathbf{7 d}$ and $\mathrm{H}$ assignation for ${ }^{1} \mathrm{H}-\mathrm{NMR}$.

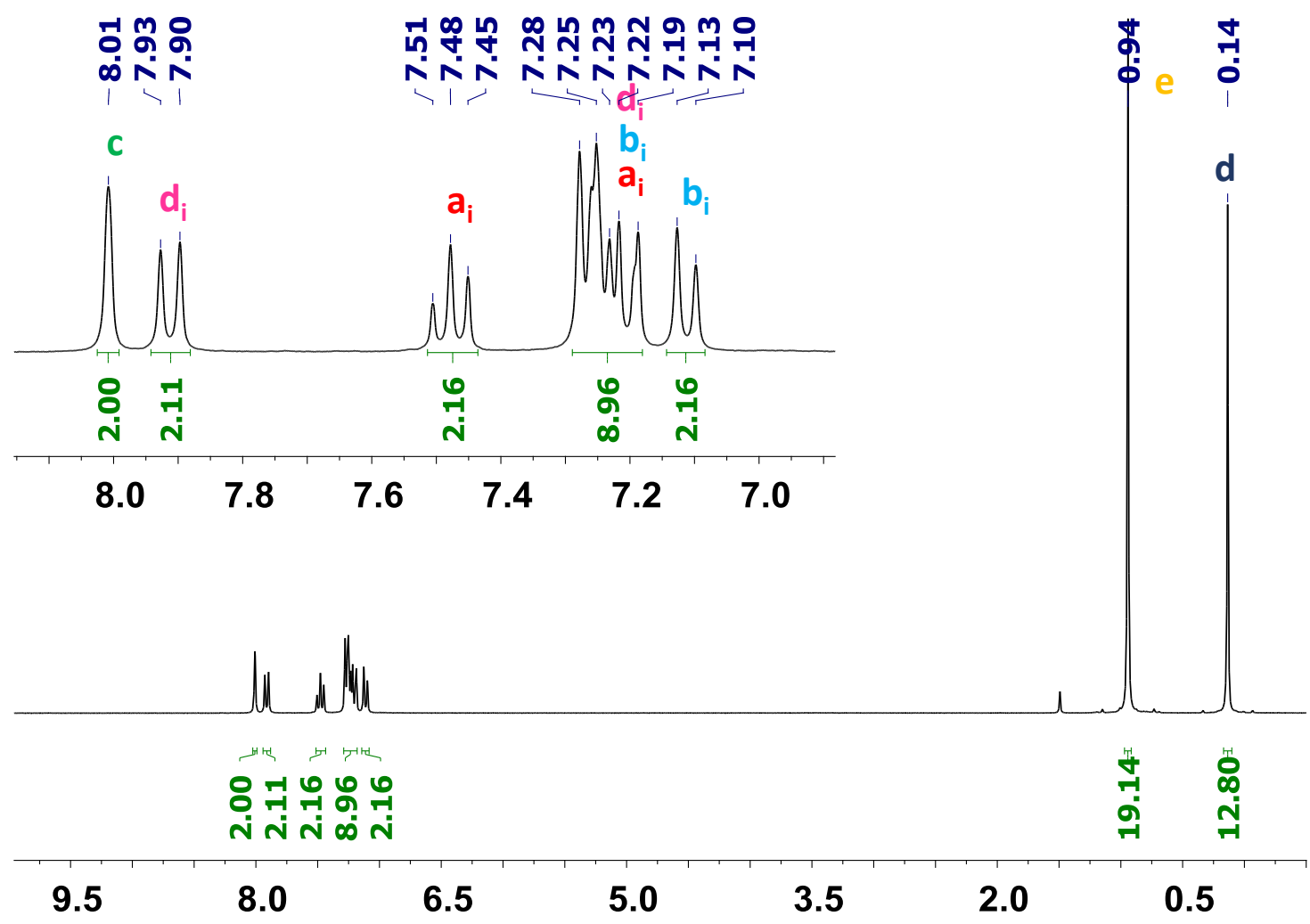

Figure S6. ${ }^{1} \mathrm{H}-\mathrm{NMR}$ of $\mathbf{7 d}$ in $\mathrm{CDCl}_{3}$, at $323 \mathrm{~K}$. 


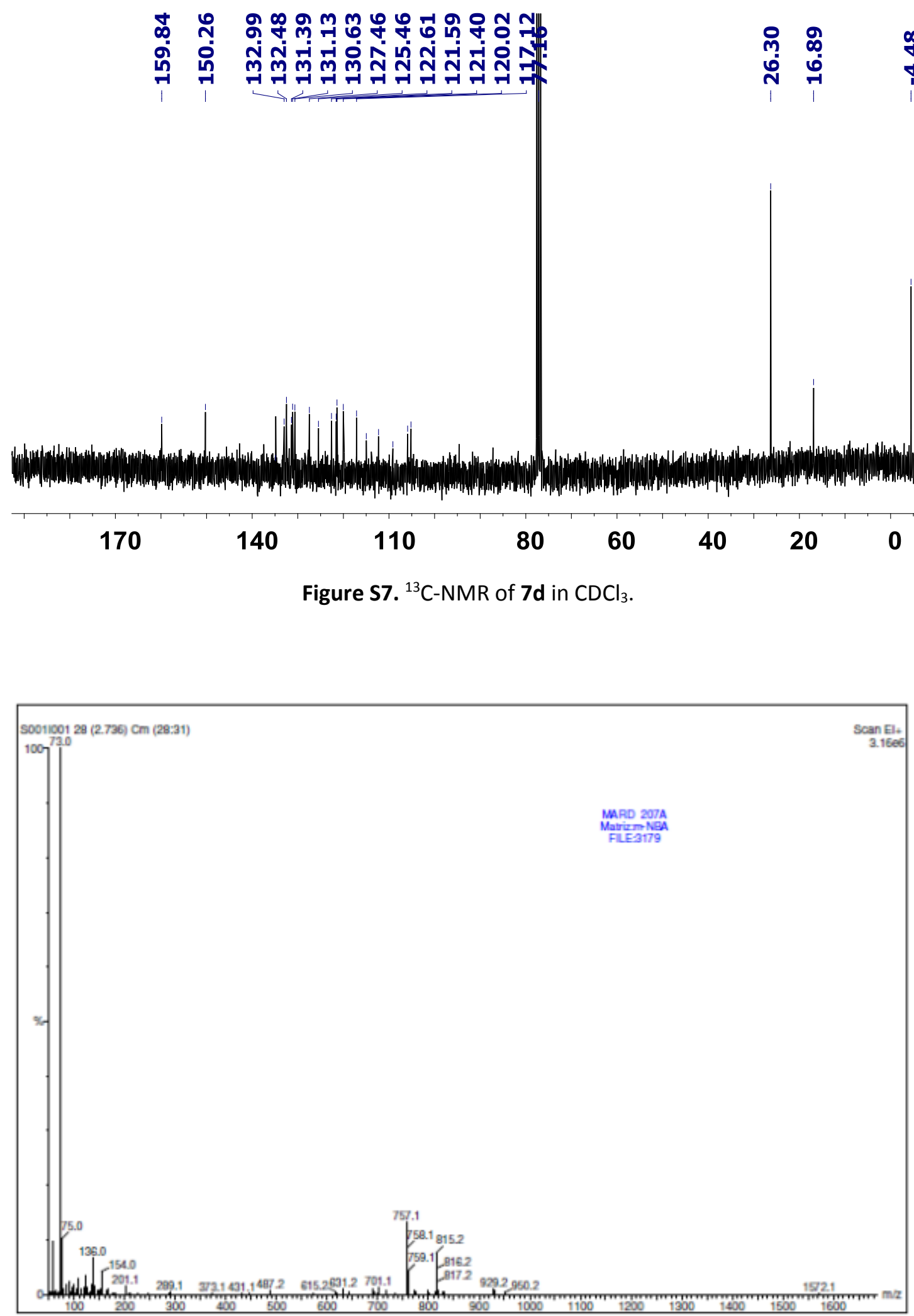



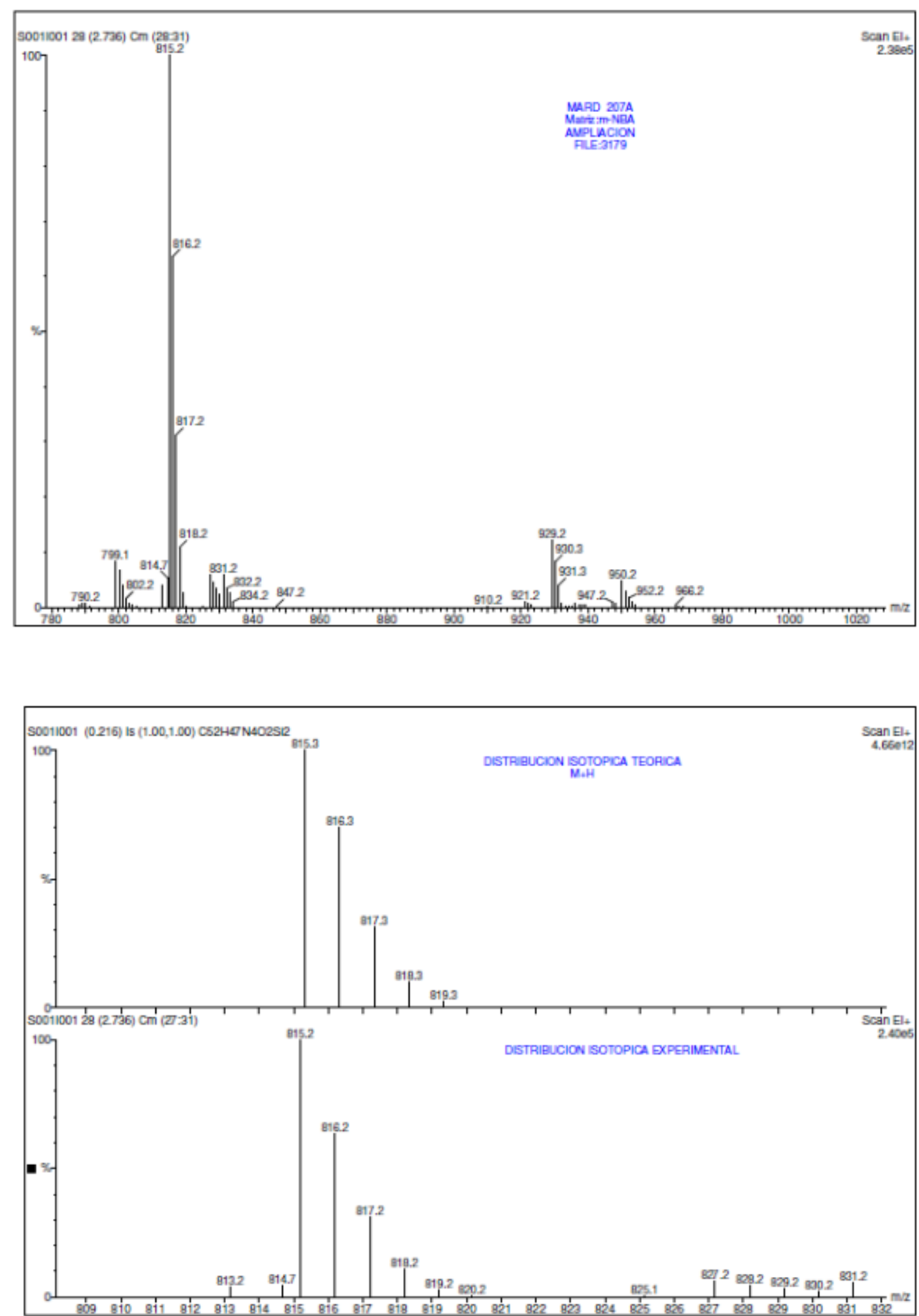


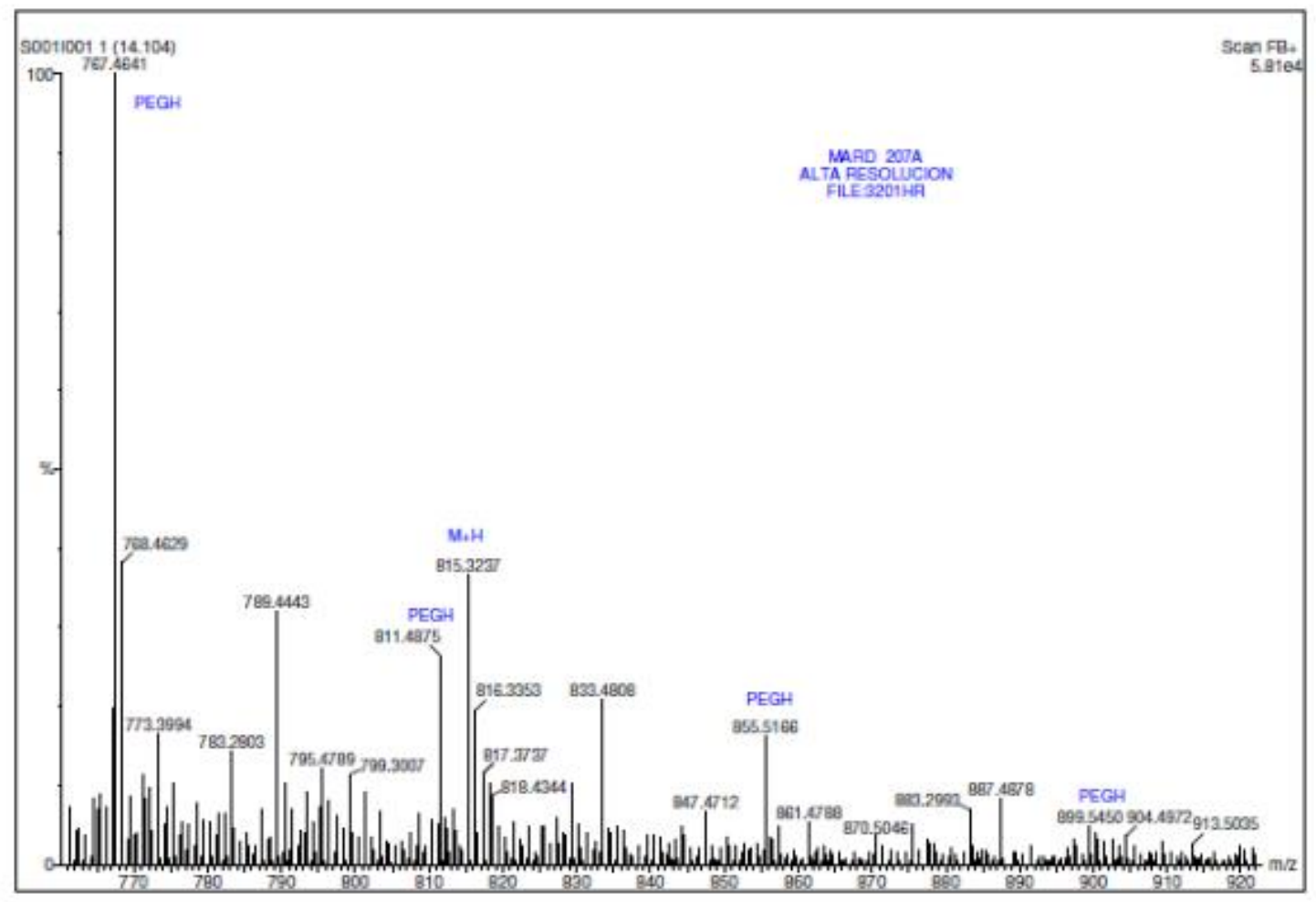

Figure S8. MS and HR-MS of 7d.

ZnPc 1

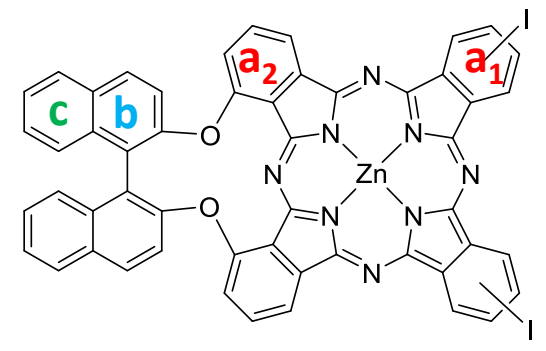

Figure 59. Structure of $\mathrm{ZnPc} 1$ and $\mathrm{H}$ assignation for ${ }^{1} \mathrm{H}-\mathrm{NMR}$. 


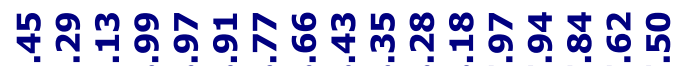
नं の
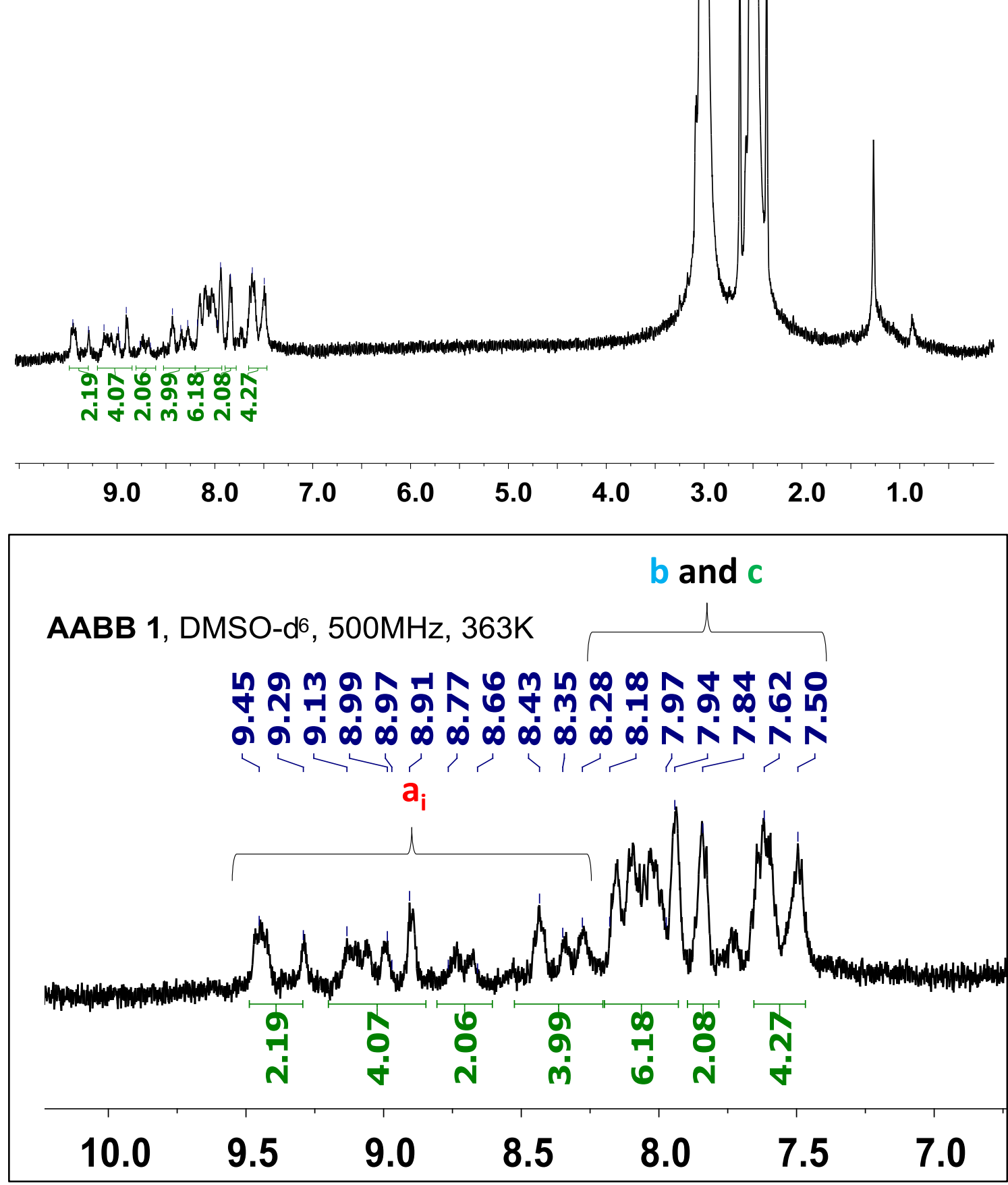

Figure S10. ${ }^{1} \mathrm{H}-\mathrm{NMR}$ and COSY of ZnPc 1 in DMSO- $\mathrm{d}_{6}$, at $363 \mathrm{~K}$ and magnification from 7 to 10 ppm. 
AABB 1, DMSO-d6, $500 \mathrm{MHz}, 298 \mathrm{~K}$

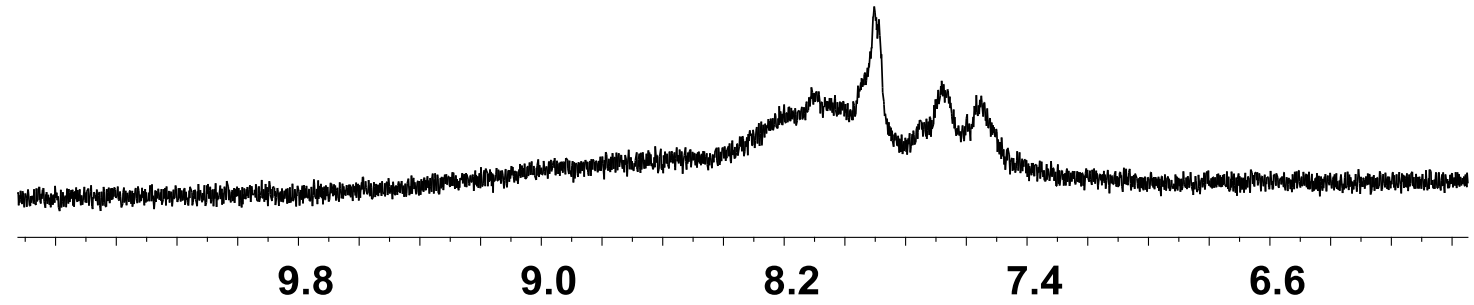

AABB 1, DMSO-d6, 500MHz, 363K

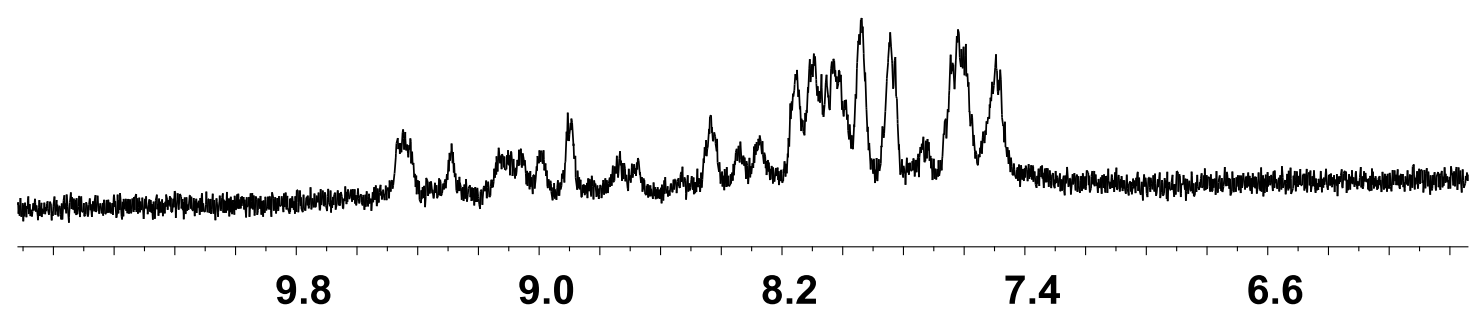

Figure S11. ${ }^{1} \mathrm{H}-\mathrm{NMR}$ variation with temperature, $298 \mathrm{~K}$ (top) and 363K (down), of ZnPc 1 in DMSO-d $d_{6}$.

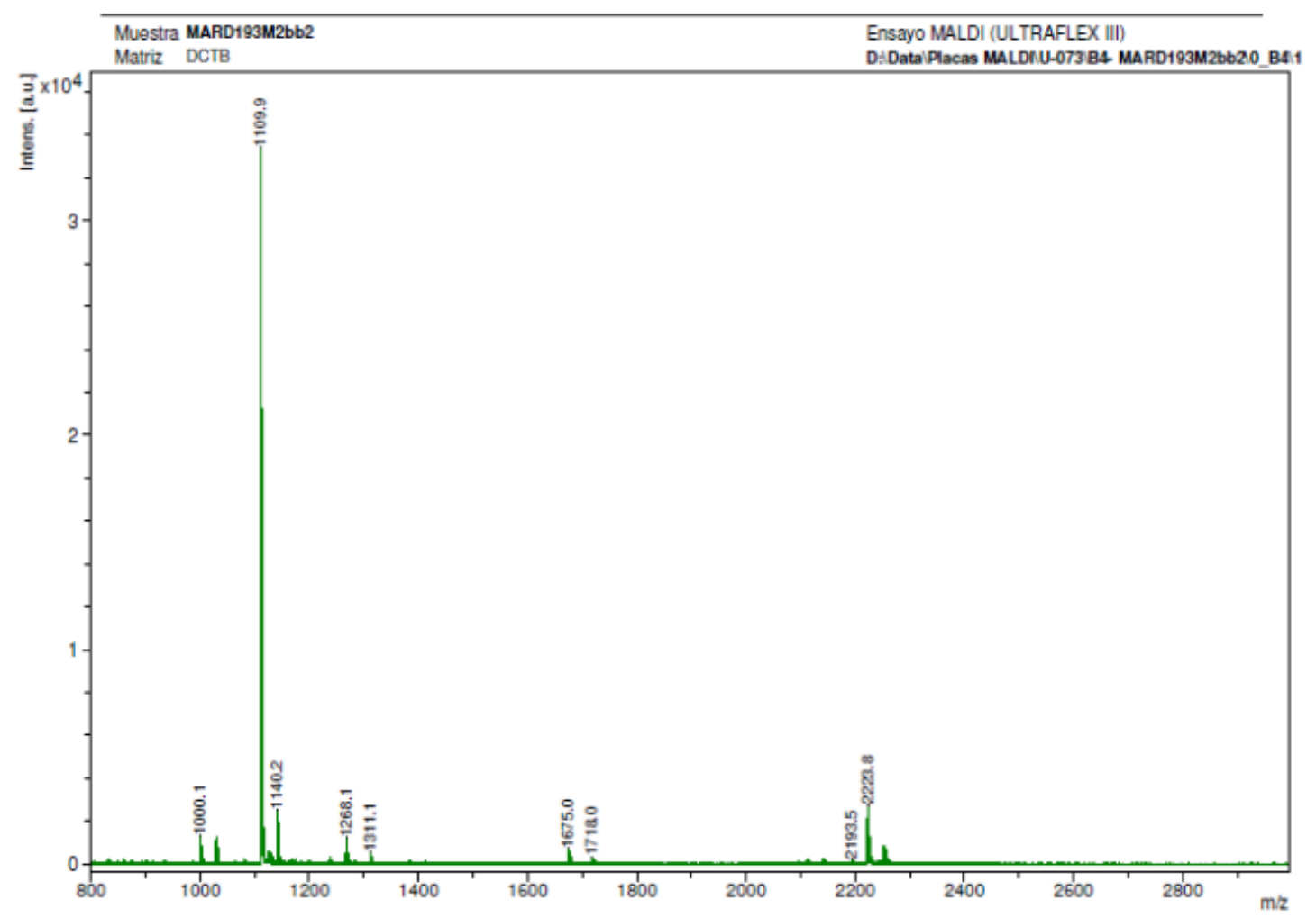




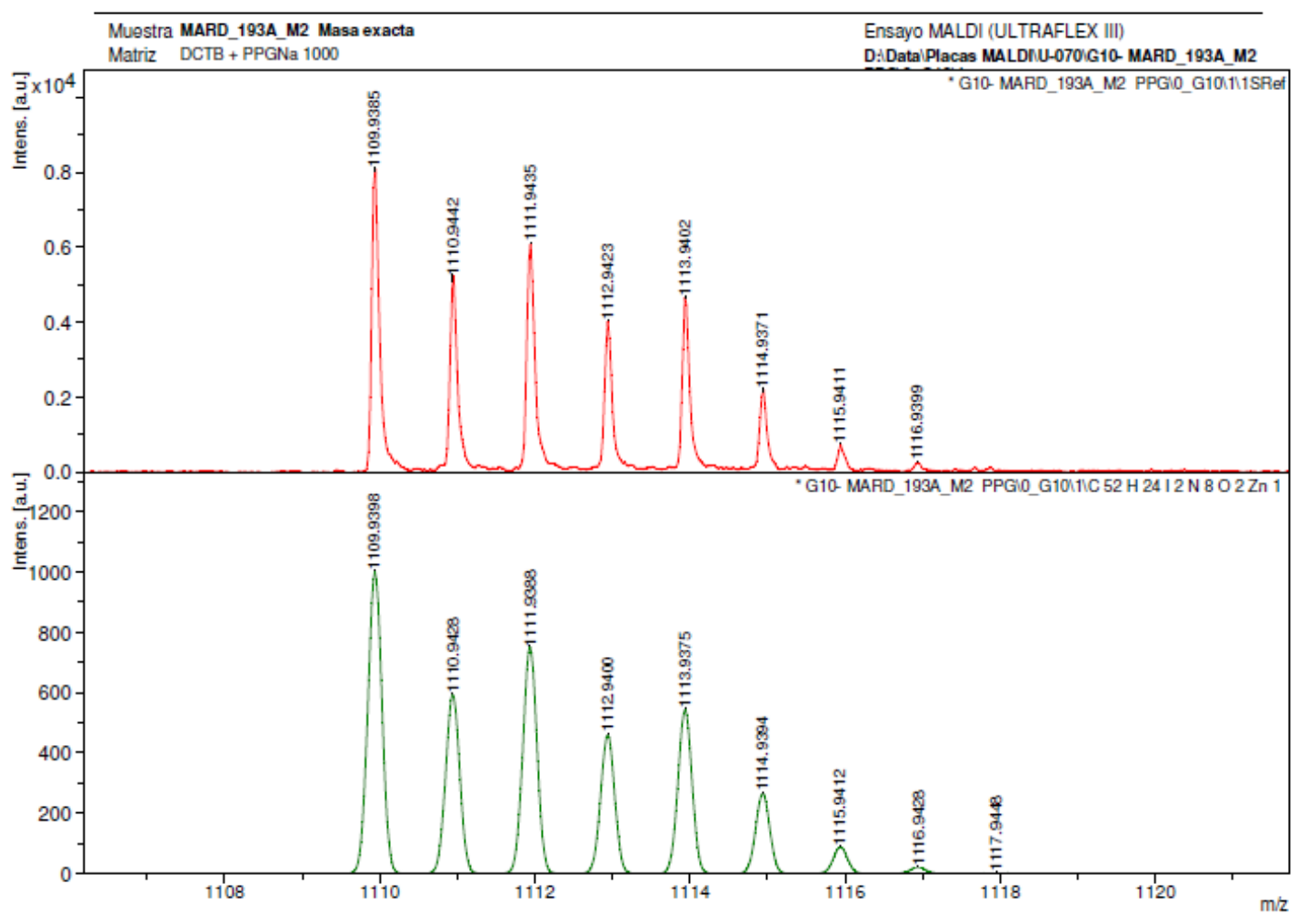

Figure S12. MS and HR-MS of ZnPc 1.

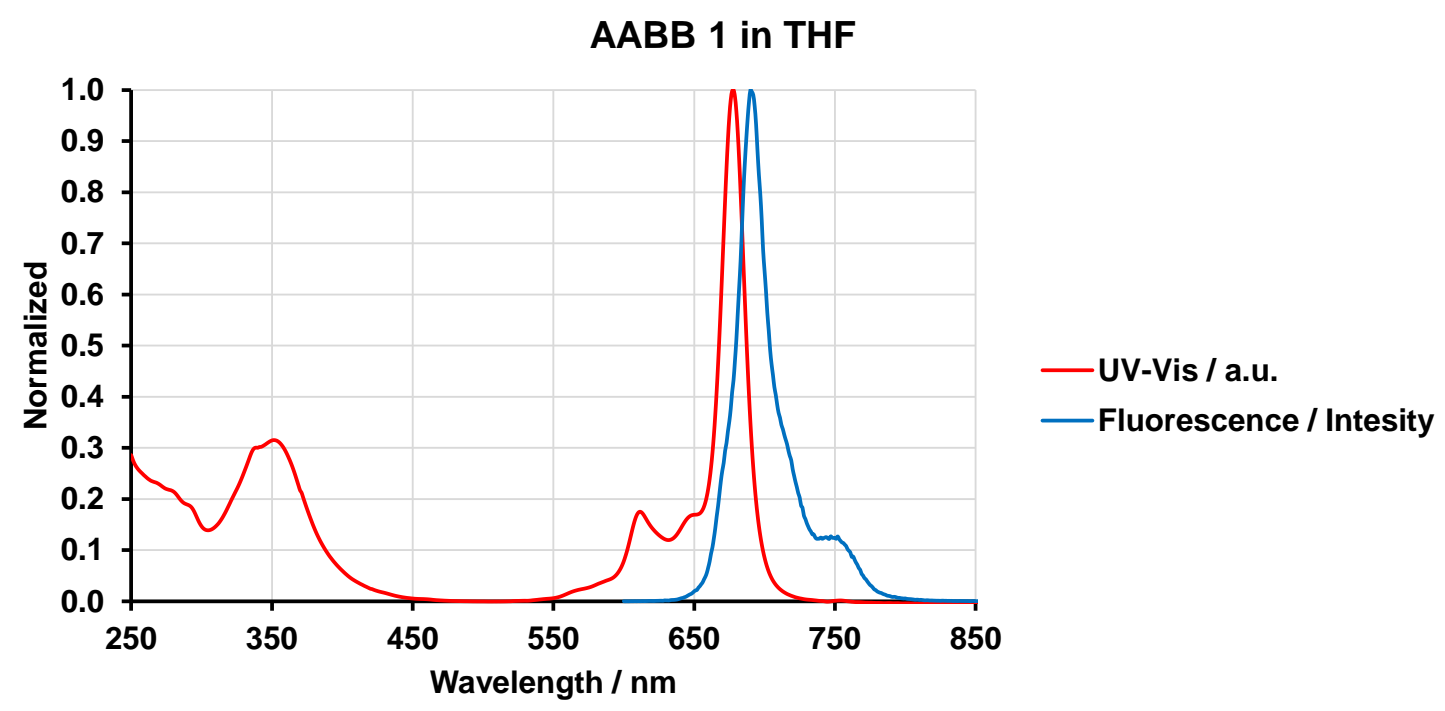

Figure S13. Normalized UV-Vis and Fluorescence spectra for ZnPc 1 in THF. 


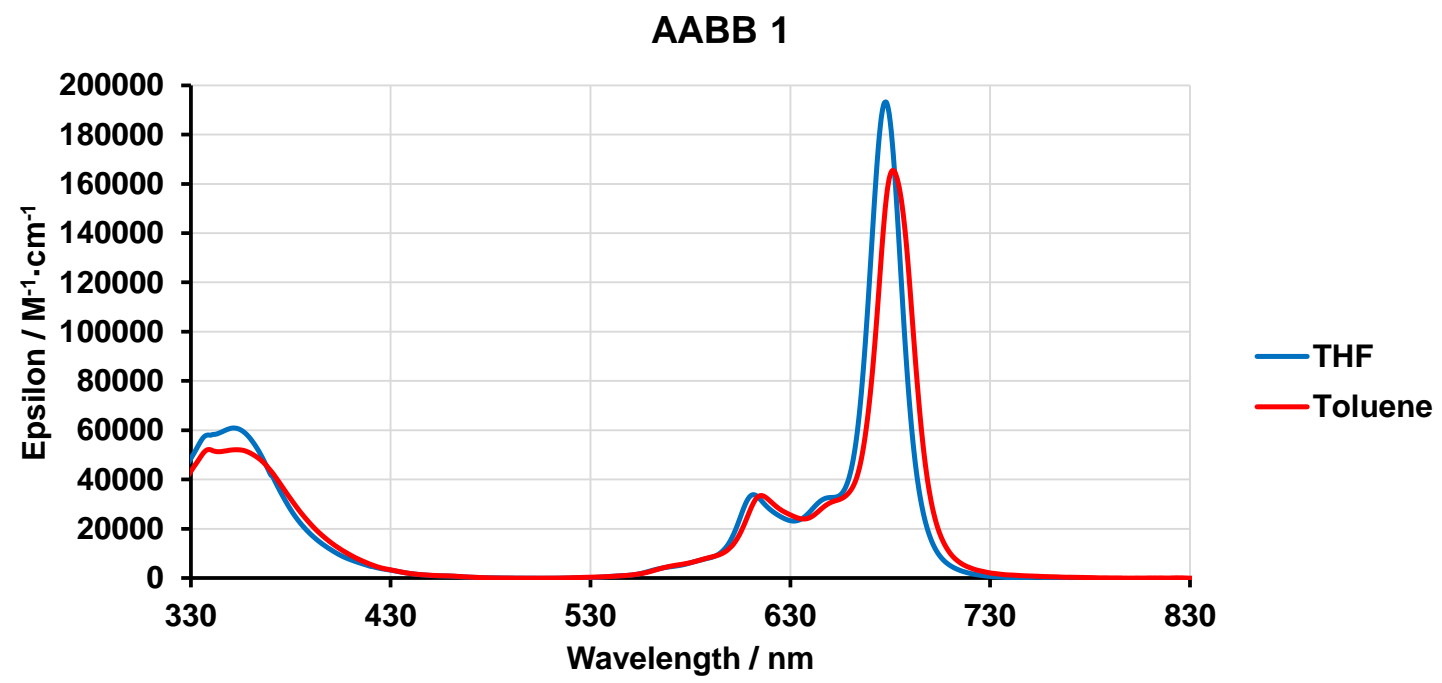

Figure S14. Normalized UV-Vis spectrum of ZnPc 1 in toluene as non-coordinative solvent and THF. Aggregation phenomena are not clearly observed.

ZnPc 2

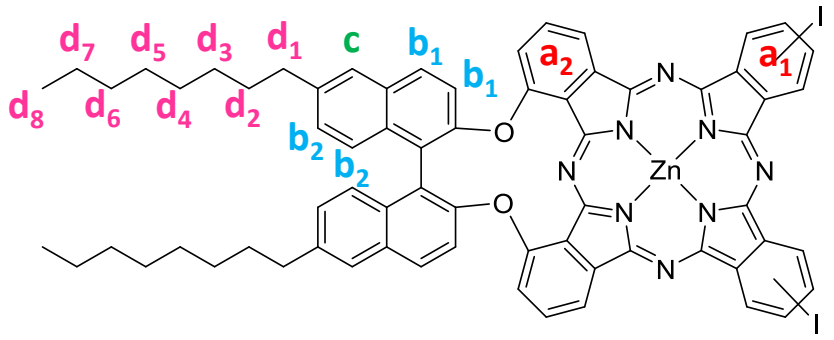

Figure S15. Structure of $\mathrm{ZnPc} 2$ and $\mathrm{H}$ assignation for ${ }^{1} \mathrm{H}-\mathrm{NMR}$. 


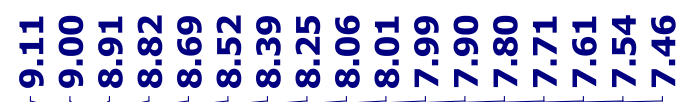

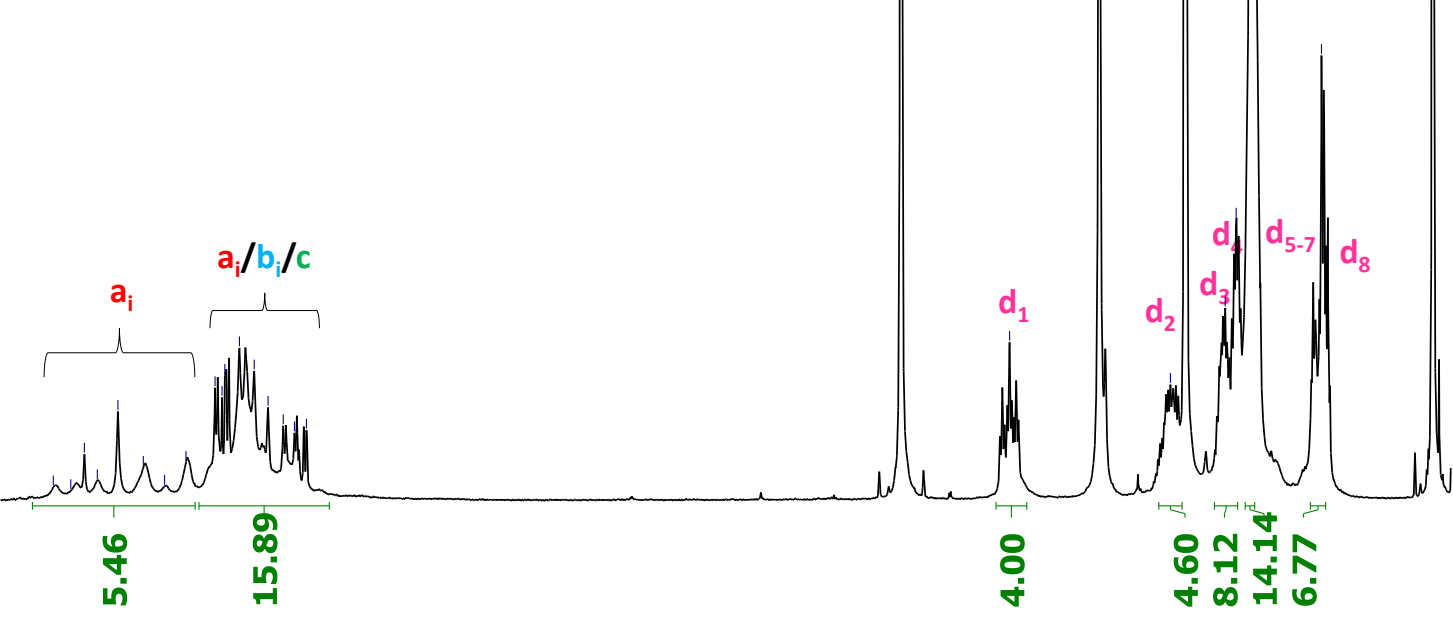

$\begin{array}{lllllllll}8.5 & 7.5 & 6.5 & 5.5 & 4.5 & 3.5 & 2.5 & 1.5 & 0.5\end{array}$

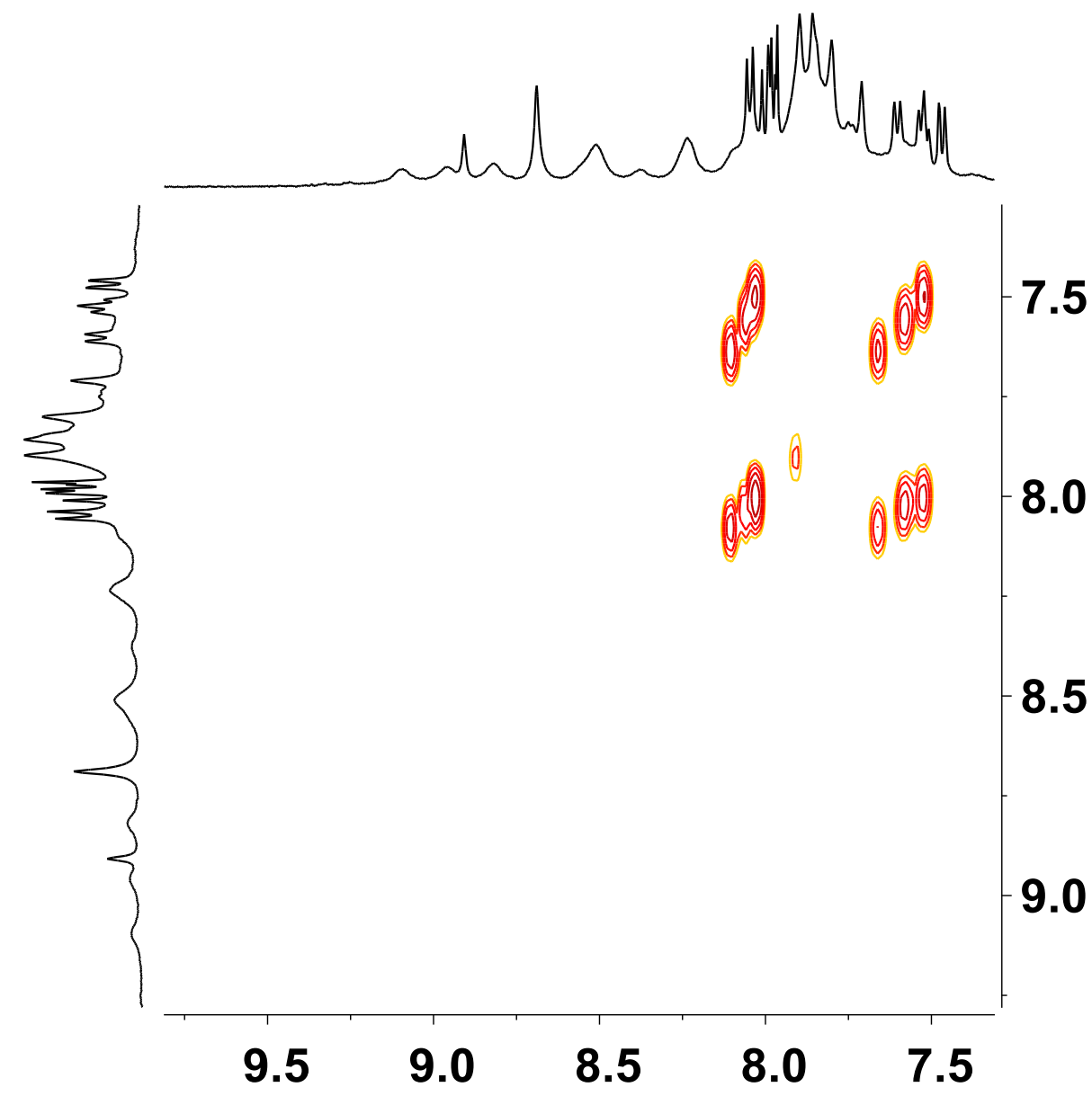




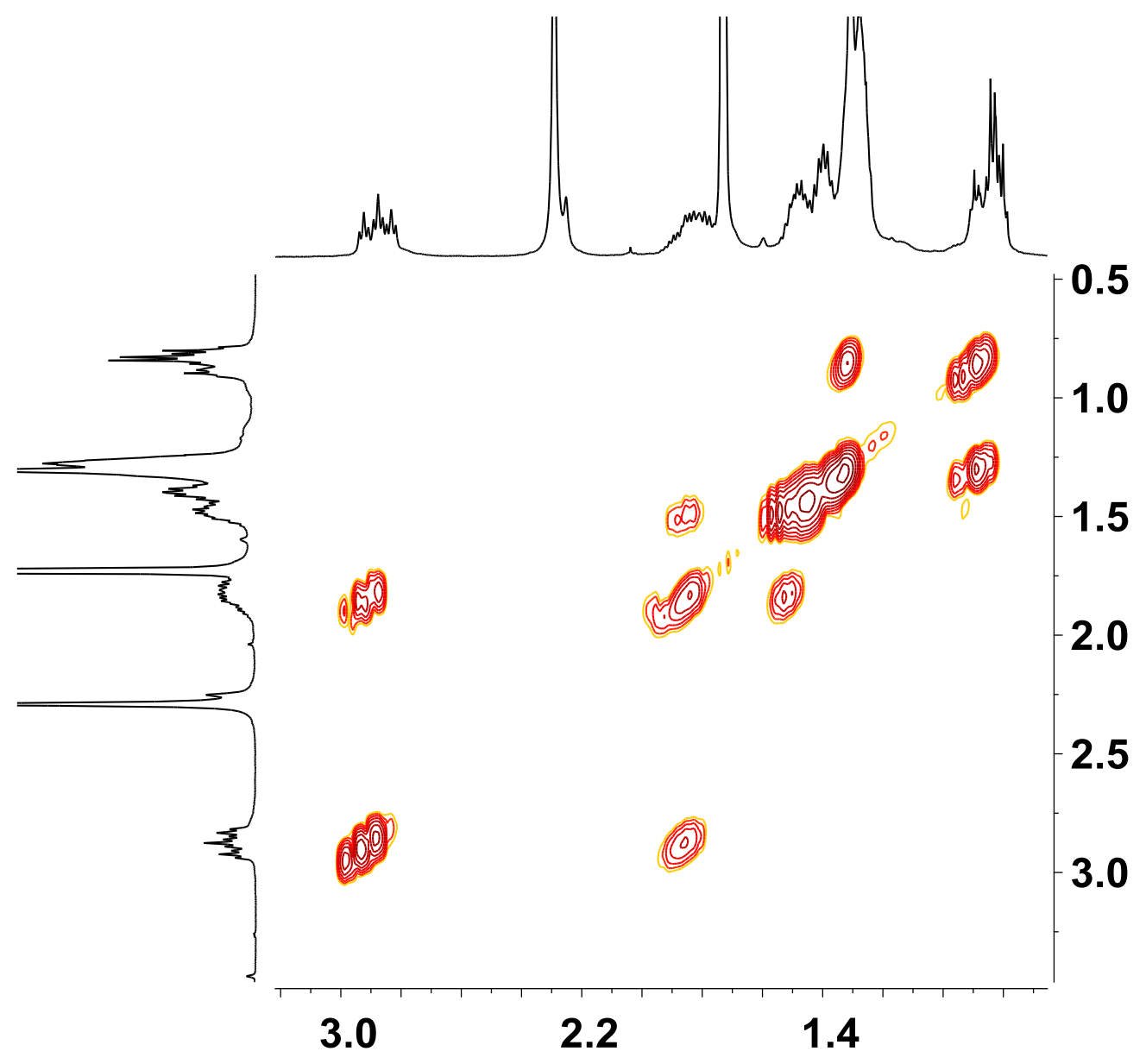

Figure S16. ${ }^{1} \mathrm{H}-\mathrm{NMR}$ and COSY of $\mathrm{ZnPc} 2$ in THF- $\mathrm{d}_{8}$, at $323 \mathrm{~K}$. 
AABB 2, THF-d8, 500MHz, 298K

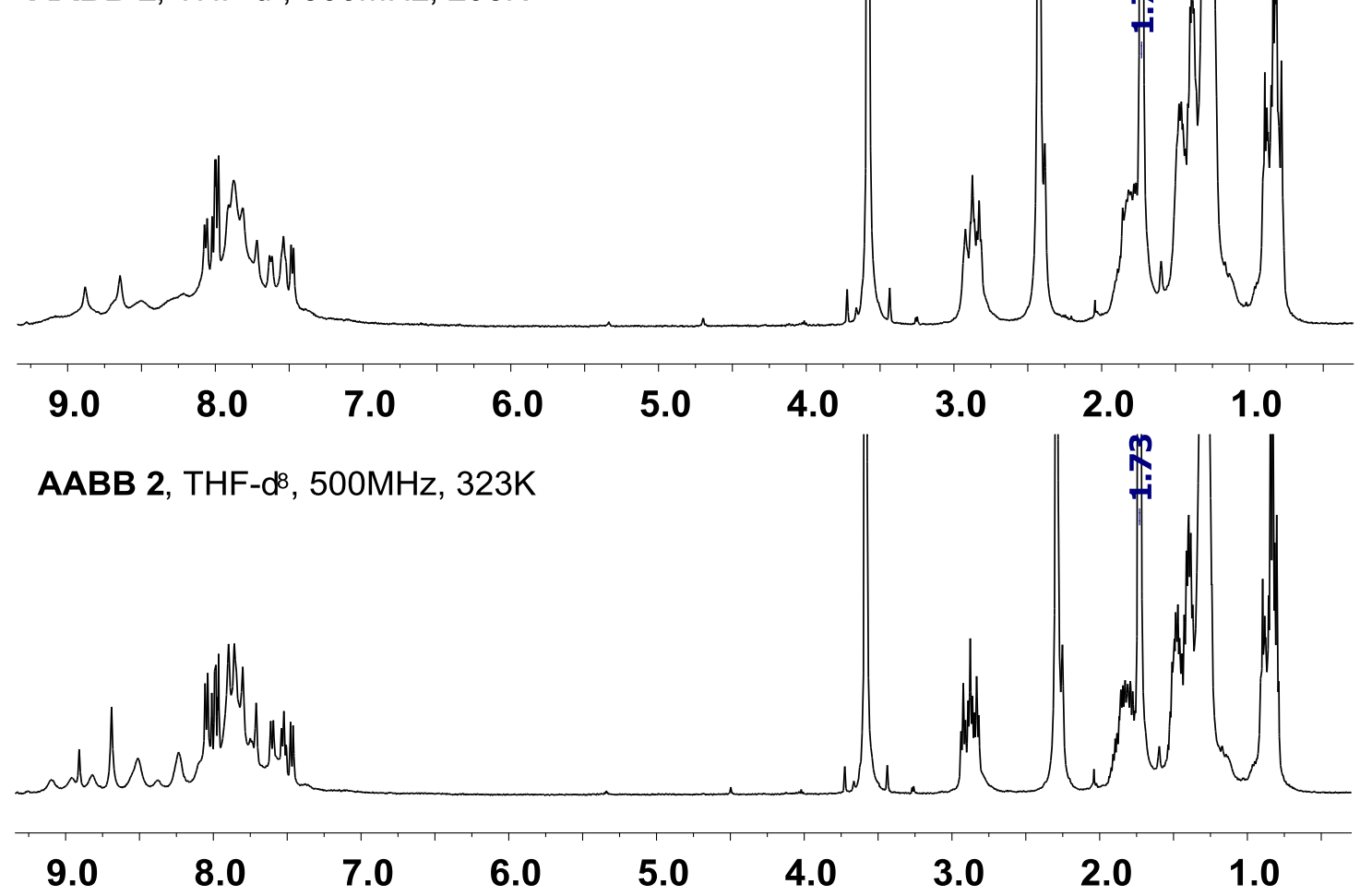

Figure S17. ${ }^{1} \mathrm{H}-\mathrm{NMR}$ variation with temperature, $298 \mathrm{~K}$ (top) and 323K (down), of ZnPc 2 in THF$d_{8}$.

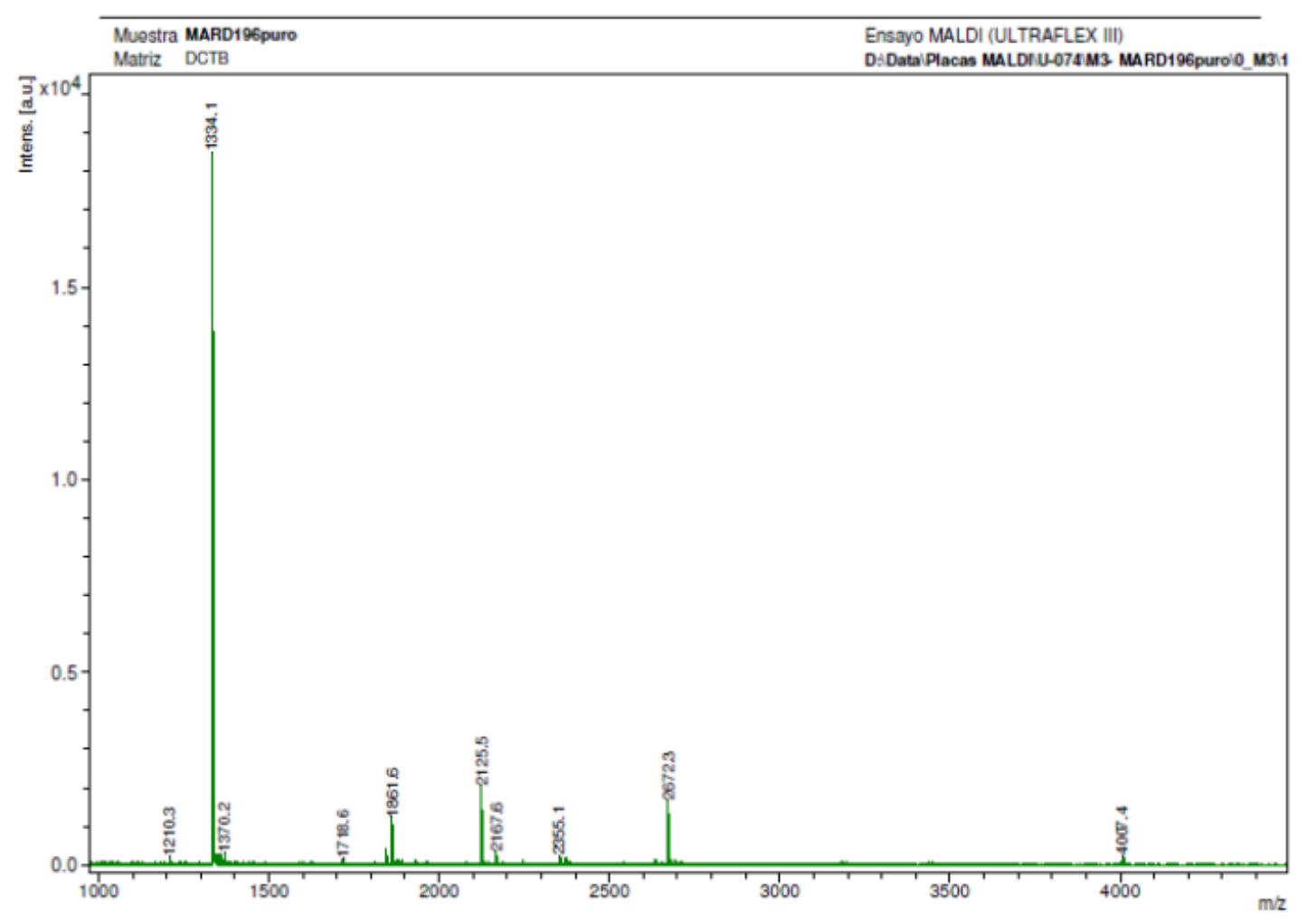




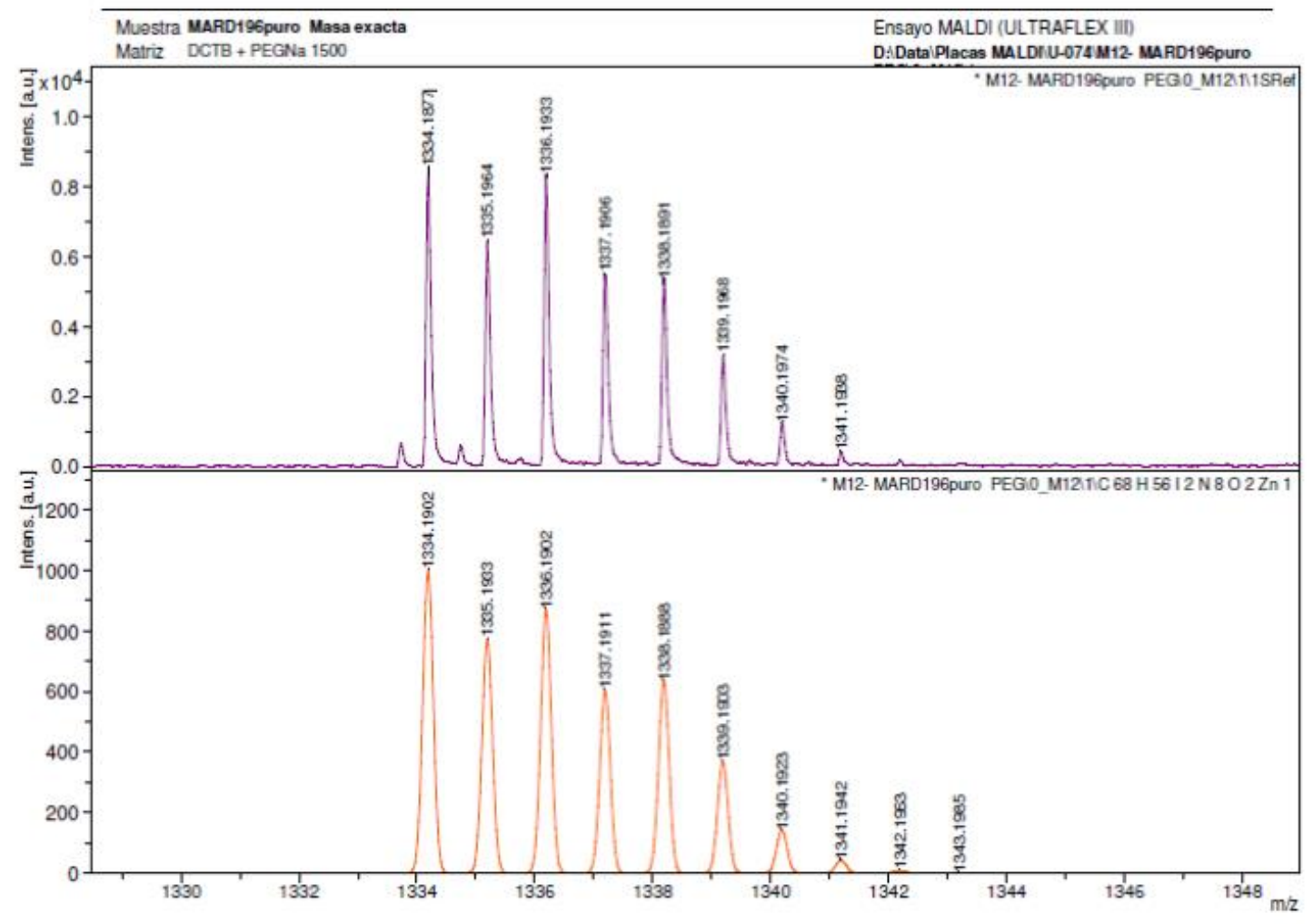

Figure S18. MS and HR-MS of ZnPc 2.

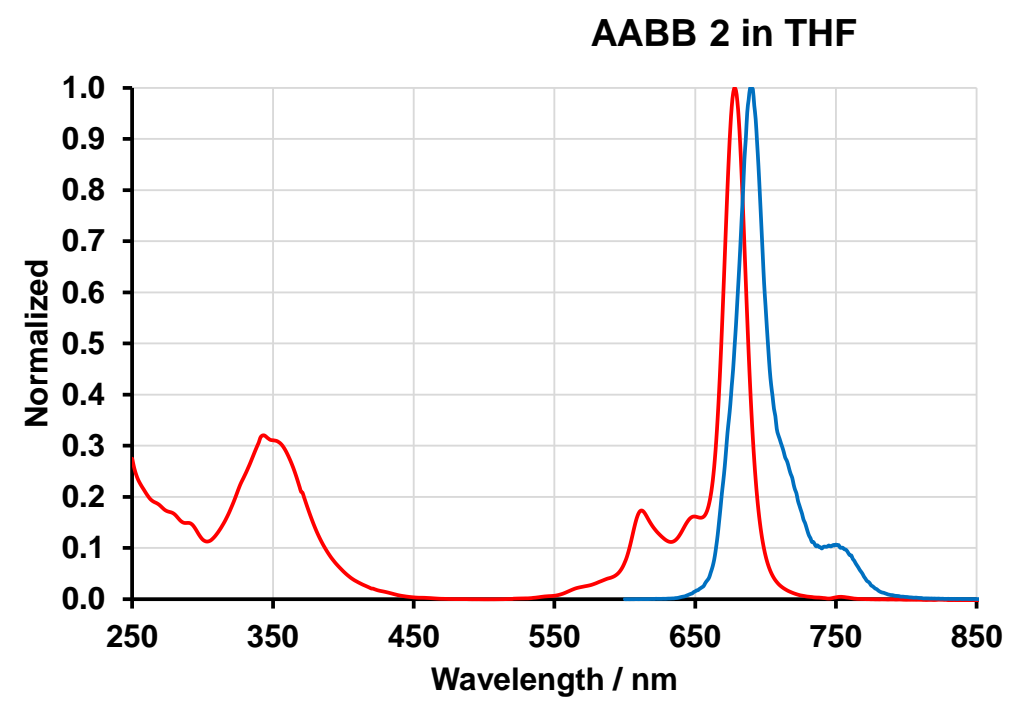

—UV-Vis / a.u.

_Fluorescence / Intesity 


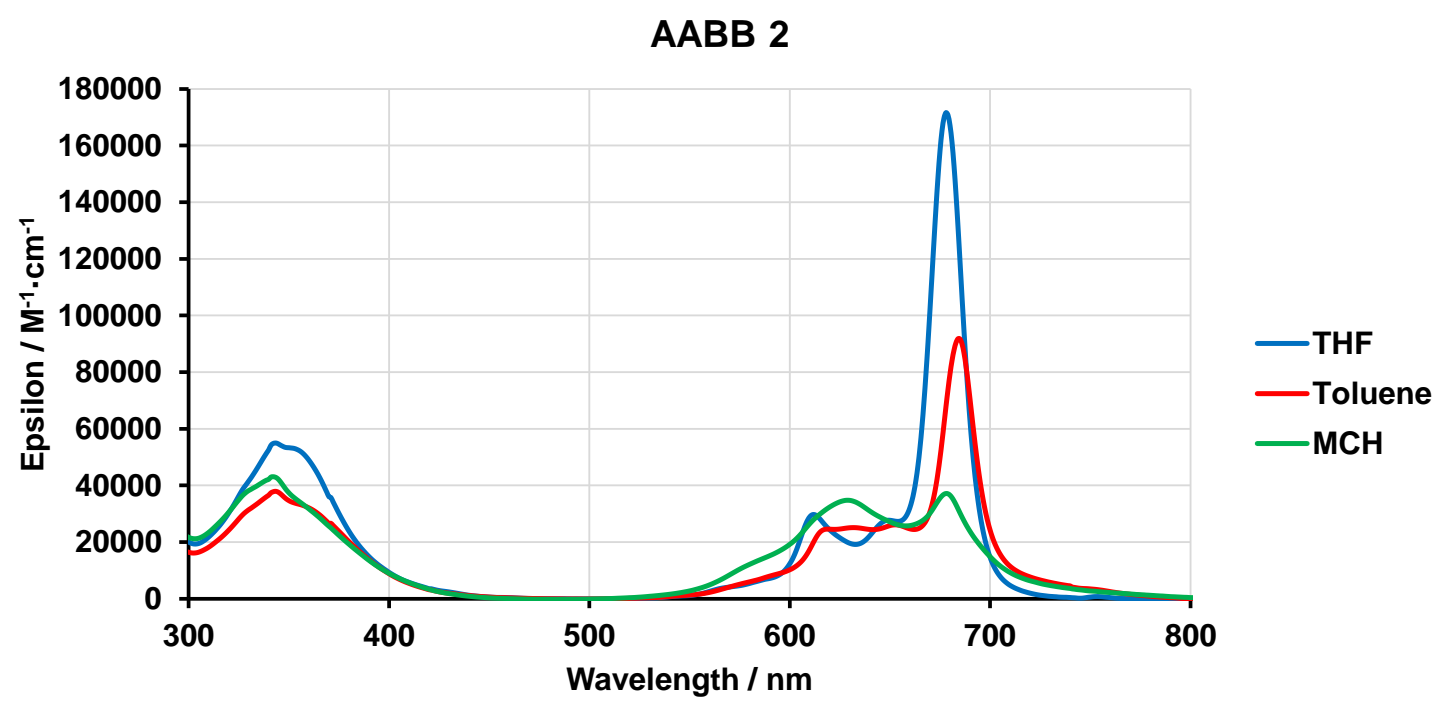

Figure S19. Normalized UV-Vis and Fluorescence spectra for ZnPc 2 in THF and normalized UVVis spectra comparison in $\mathrm{THF}$, toluene and $\mathrm{MCH}$.

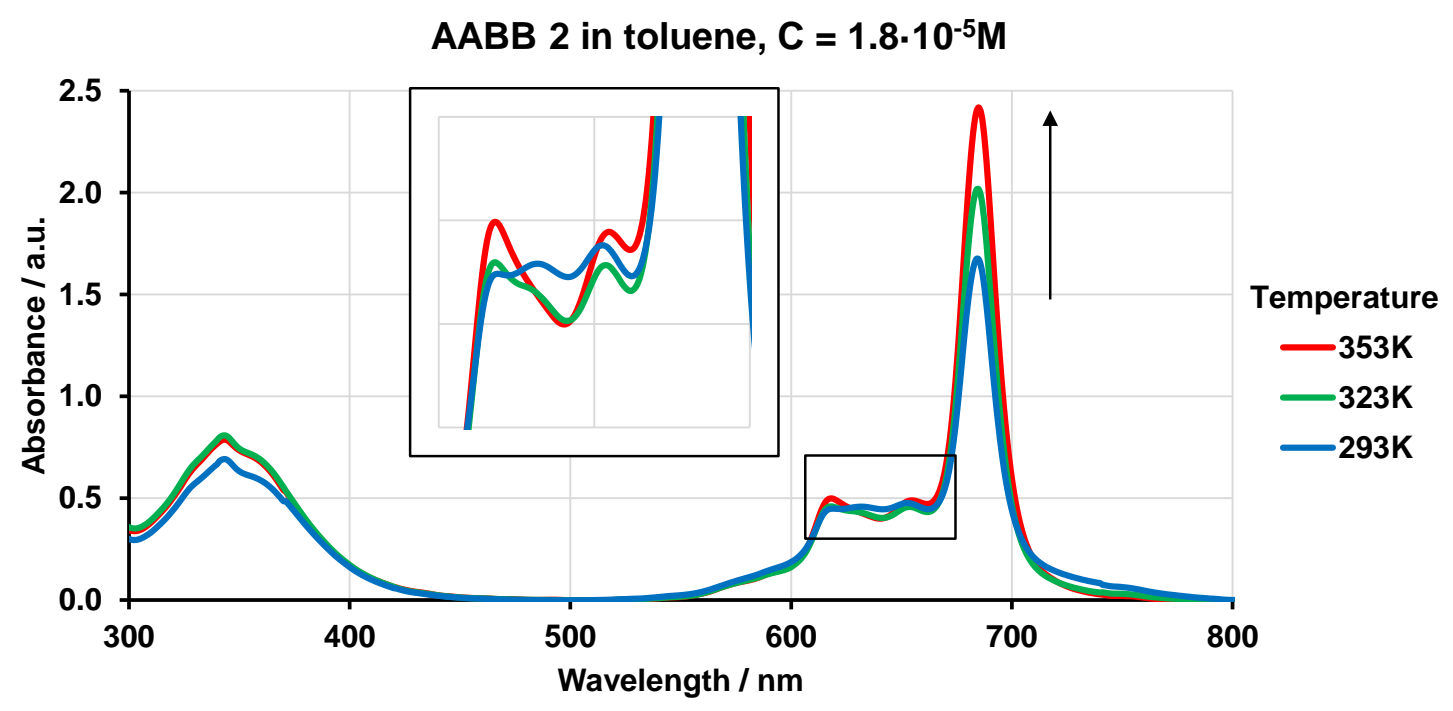



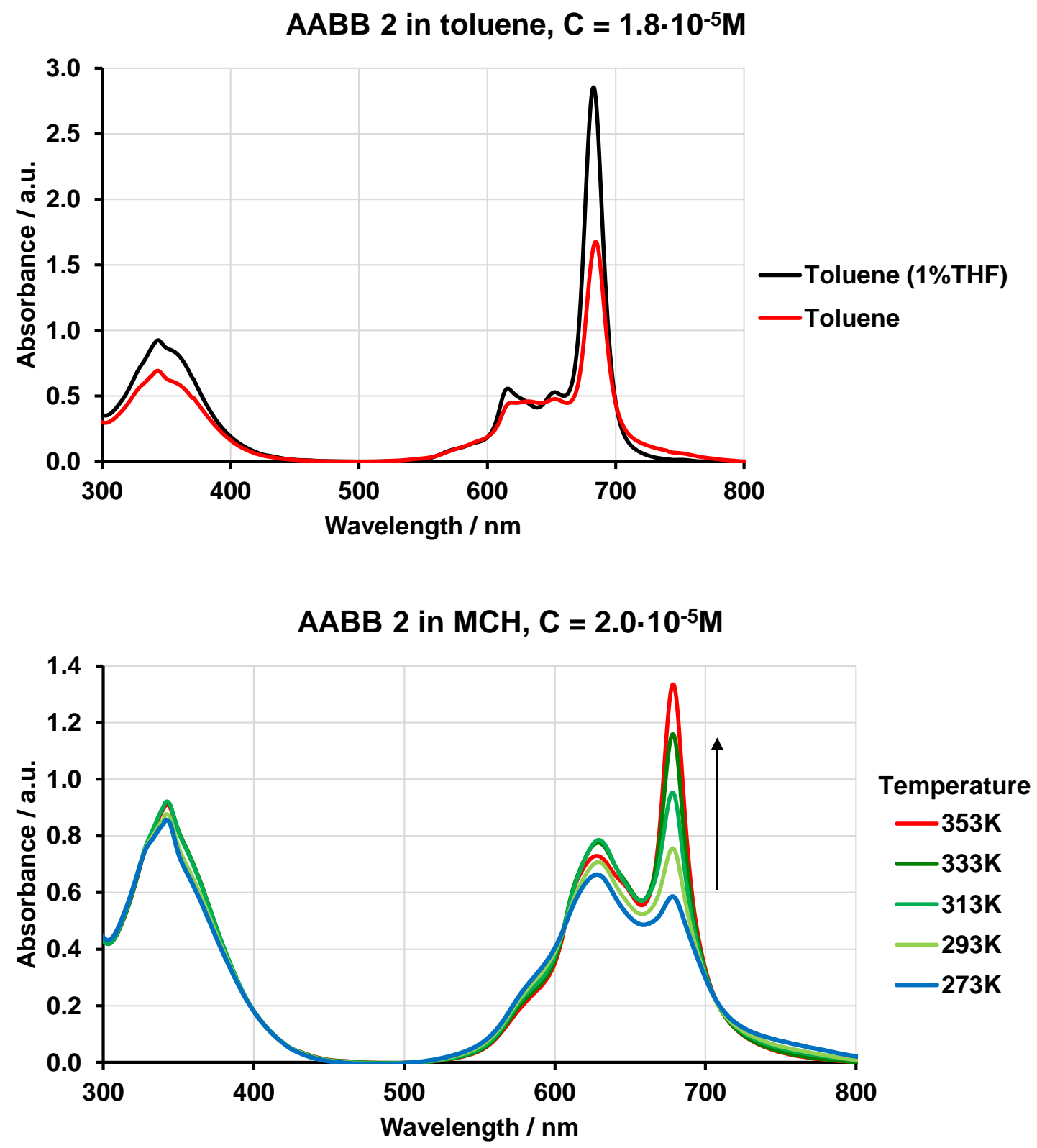


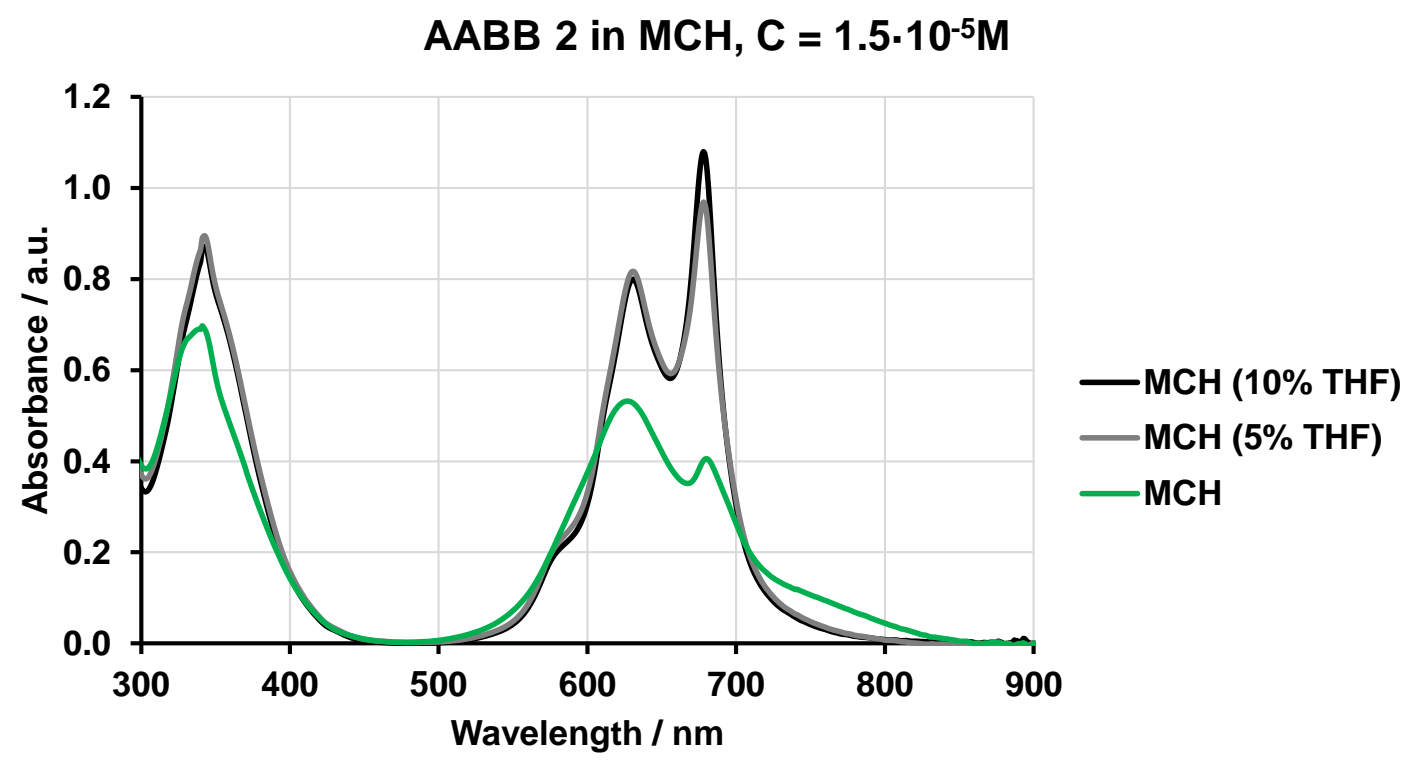

Figure S20. Disaggregation of AABB 2 in toluene and $\mathrm{MCH}$ with temperature and upon addition of THF. 

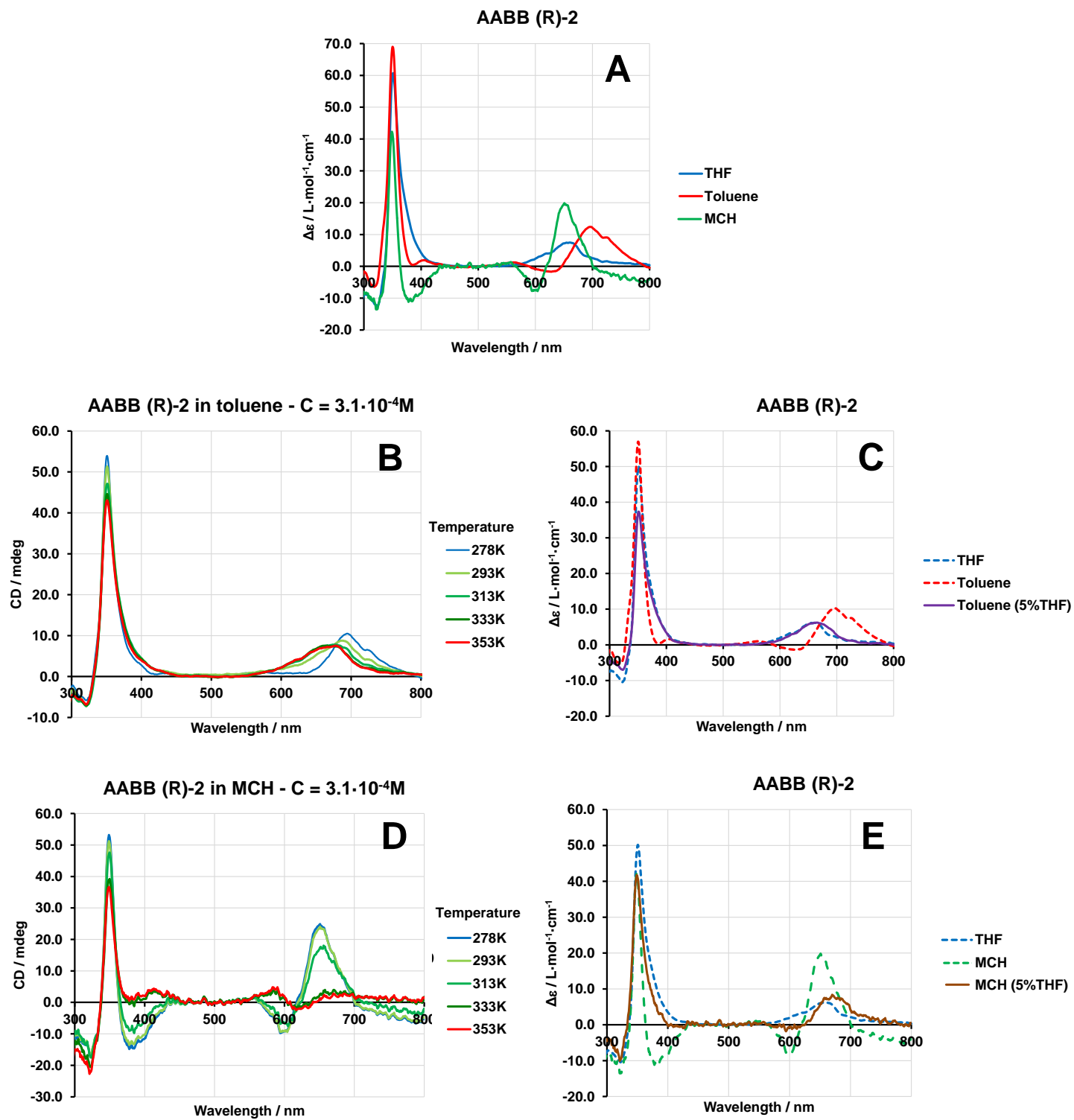

Figure S21. A) Normalized CD of ZnPc $\mathbf{2}$ in THF, toluene and MCH. B and D) Temperature dependence in toluene and $\mathrm{MCH}$. $\mathrm{C}$ and $\mathrm{E}$ ) Disaggregation upon addition of THF over toluene and $\mathrm{MCH}$ solutions.

\section{ZnPc 3}

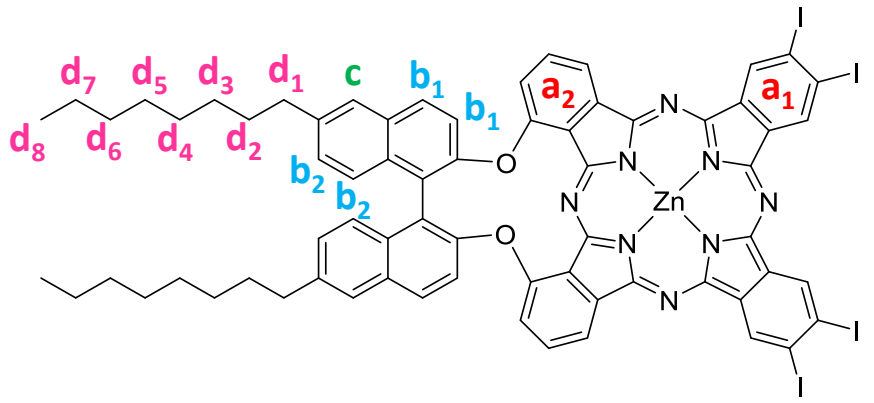

Figure S22. Structure of $\mathrm{ZnPc} 3$ and $\mathrm{H}$ assignation for ${ }^{1} \mathrm{H}-\mathrm{NMR}$. 


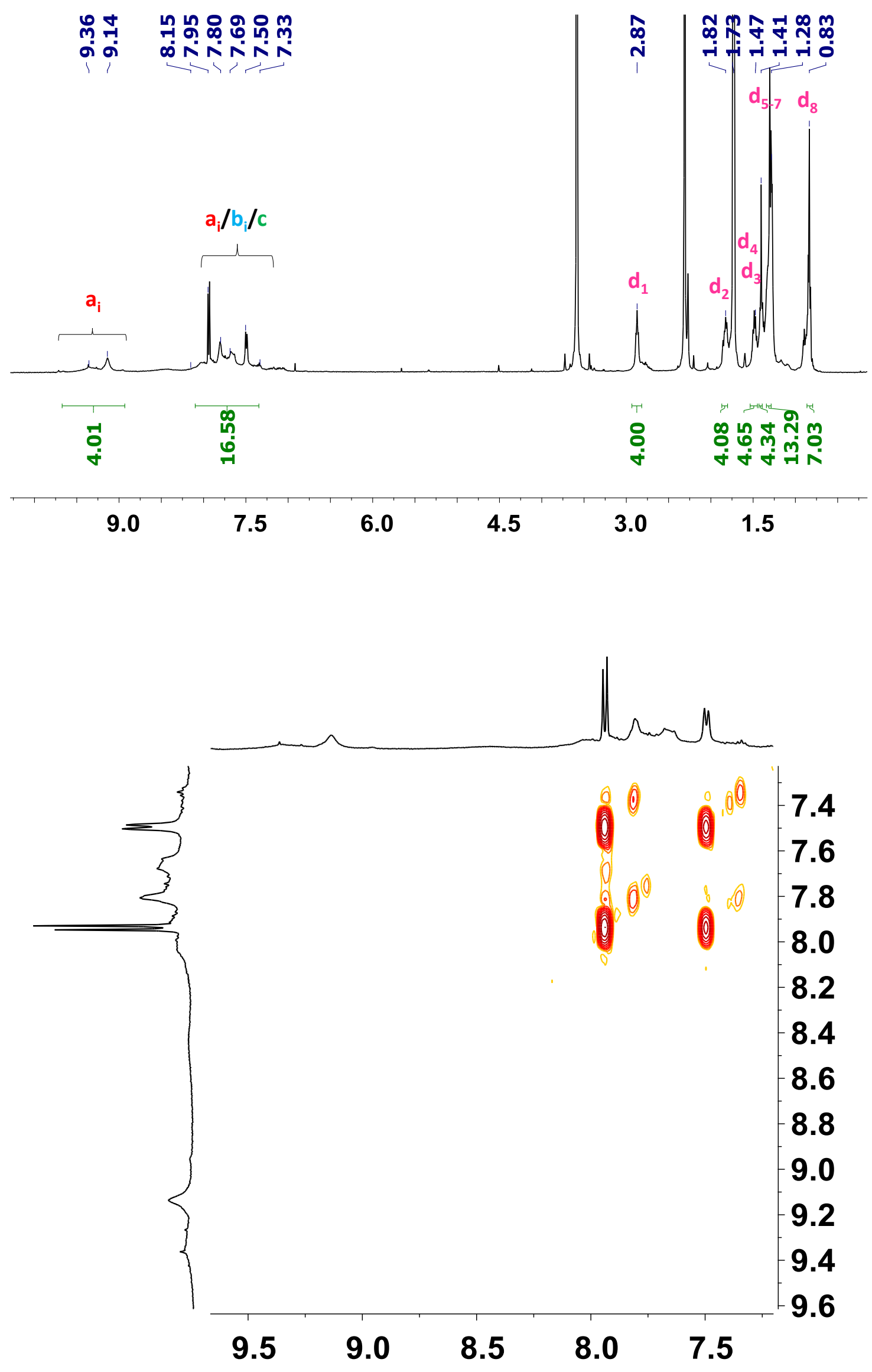




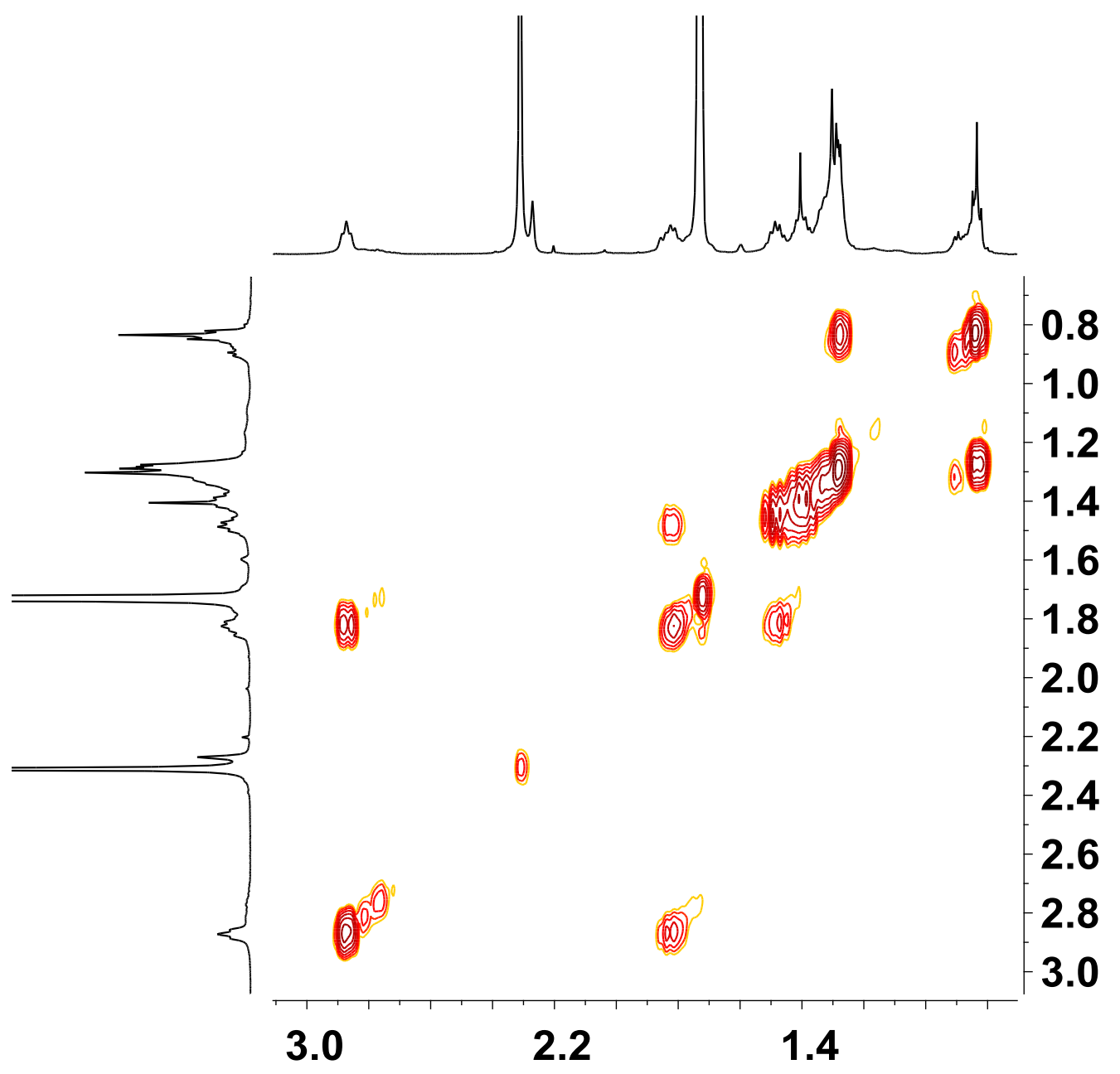

Figure S23. ${ }^{1} \mathrm{H}-\mathrm{NMR}$ and $\mathrm{COSY}$ of $\mathrm{ZnPc} 3$ in THF- $\mathrm{d}_{8}$, at $323 \mathrm{~K}$. 


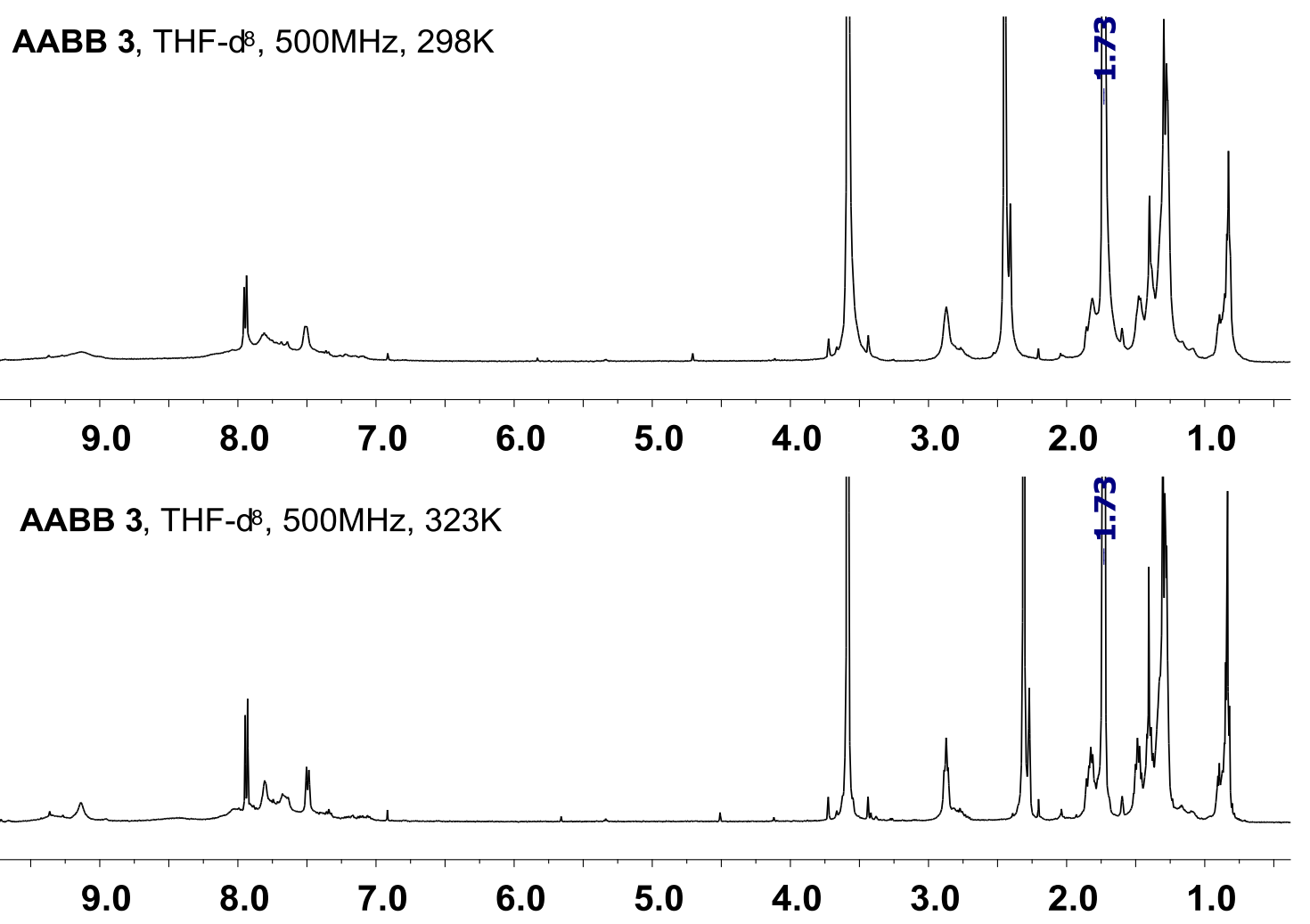

Figure S24. ${ }^{1} \mathrm{H}-\mathrm{NMR}$ variation with temperature, $298 \mathrm{~K}$ (top) and $323 \mathrm{~K}$ (down), of $\mathrm{ZnPc} 3$ in THF$d_{8}$.

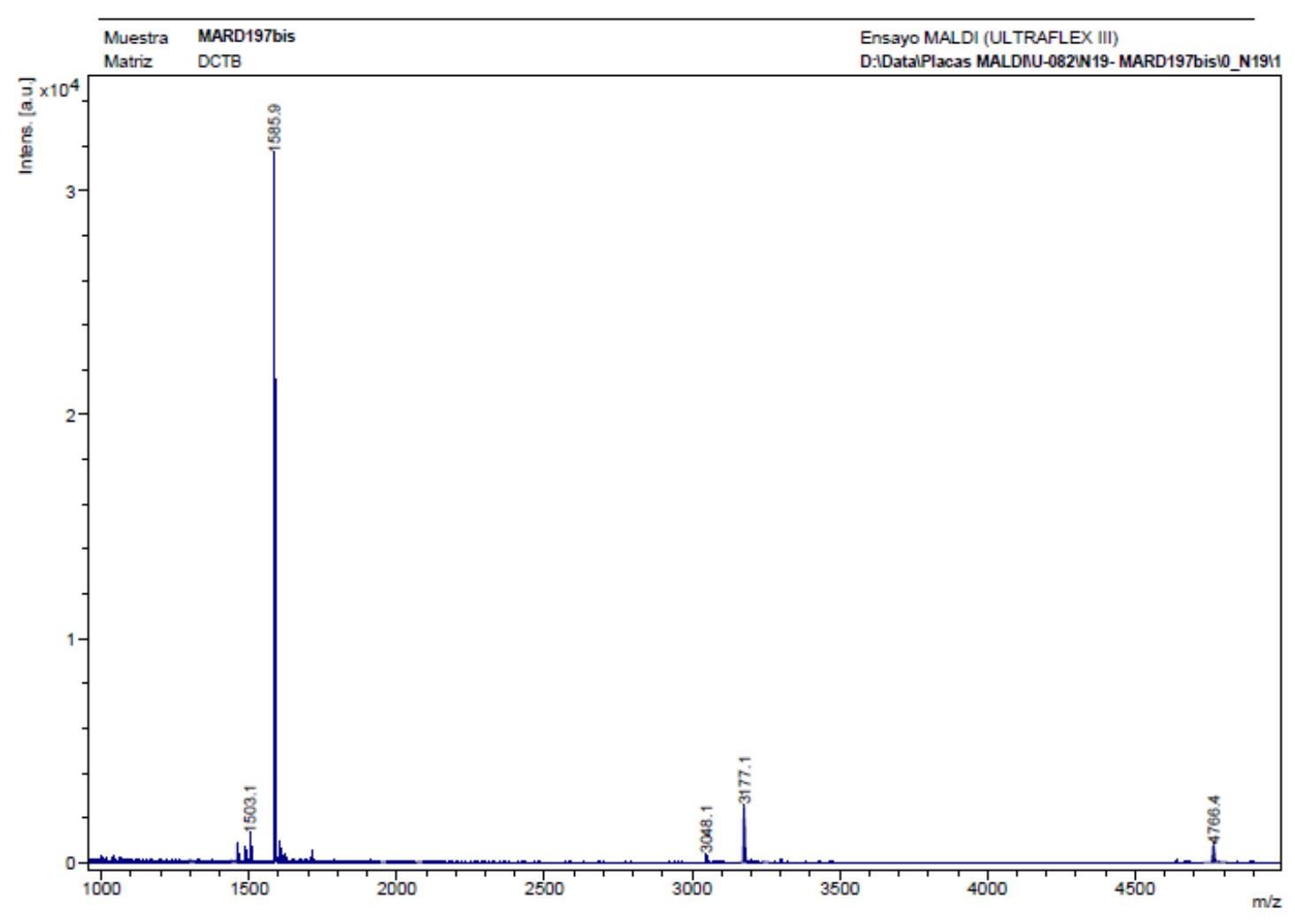




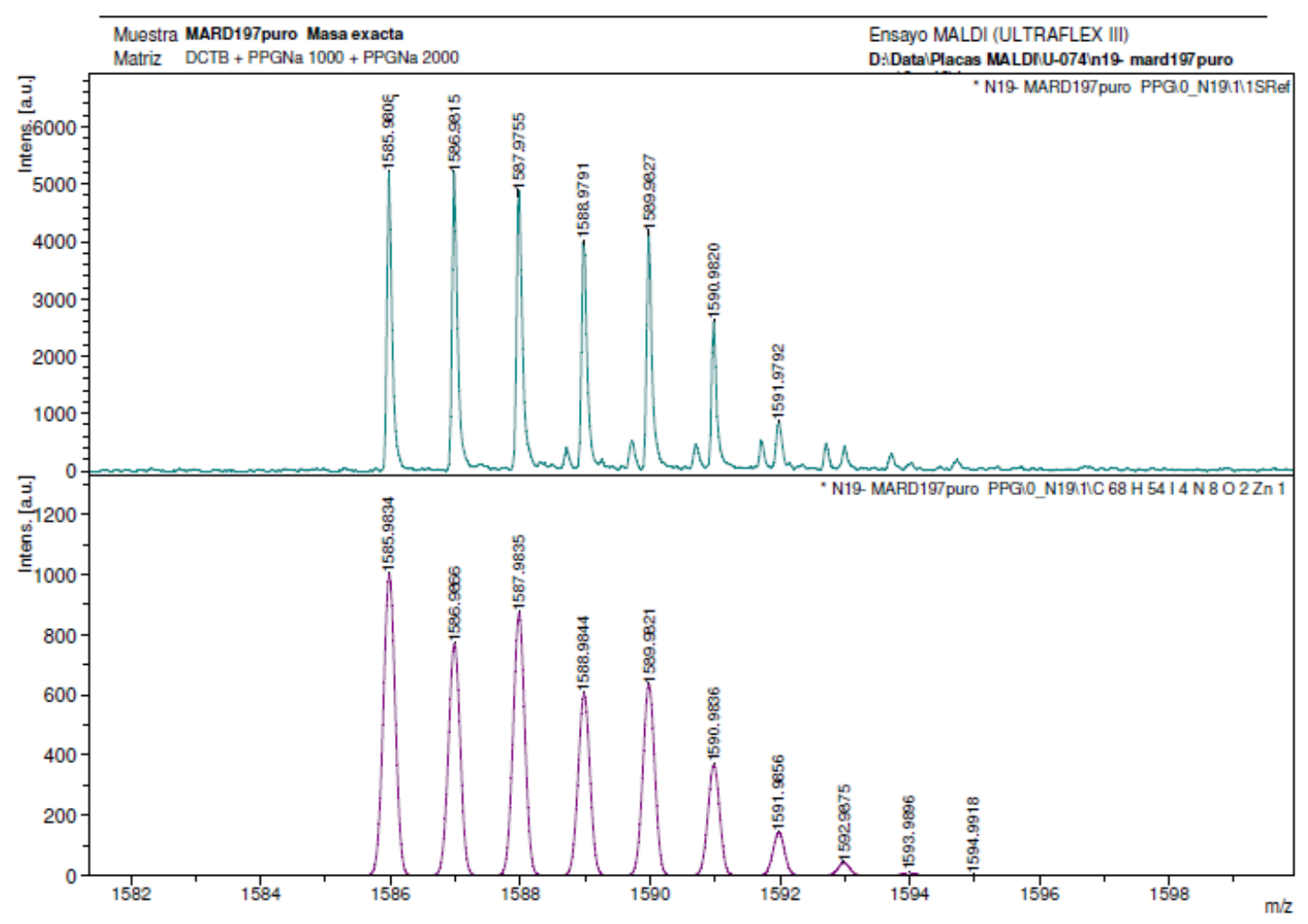

Figure S25. MS and HR-MS of ZnPc 3.

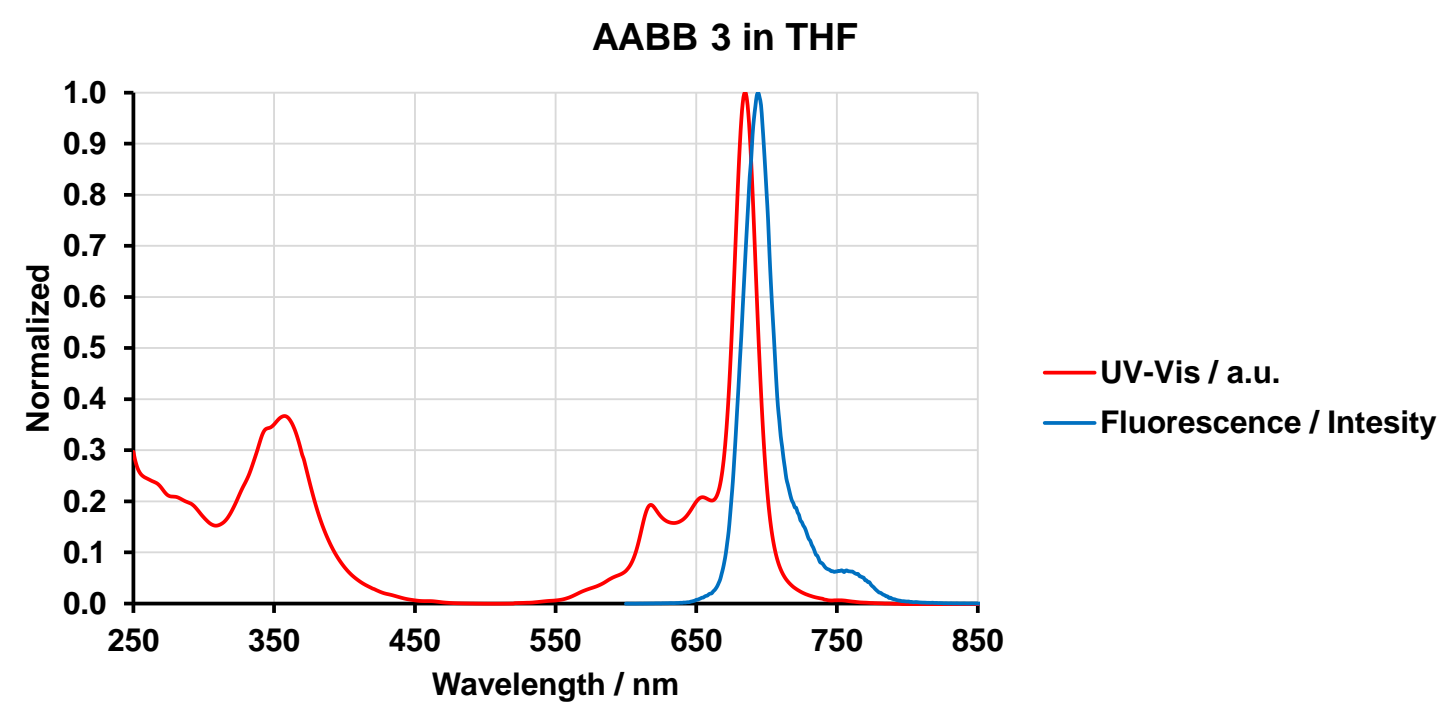




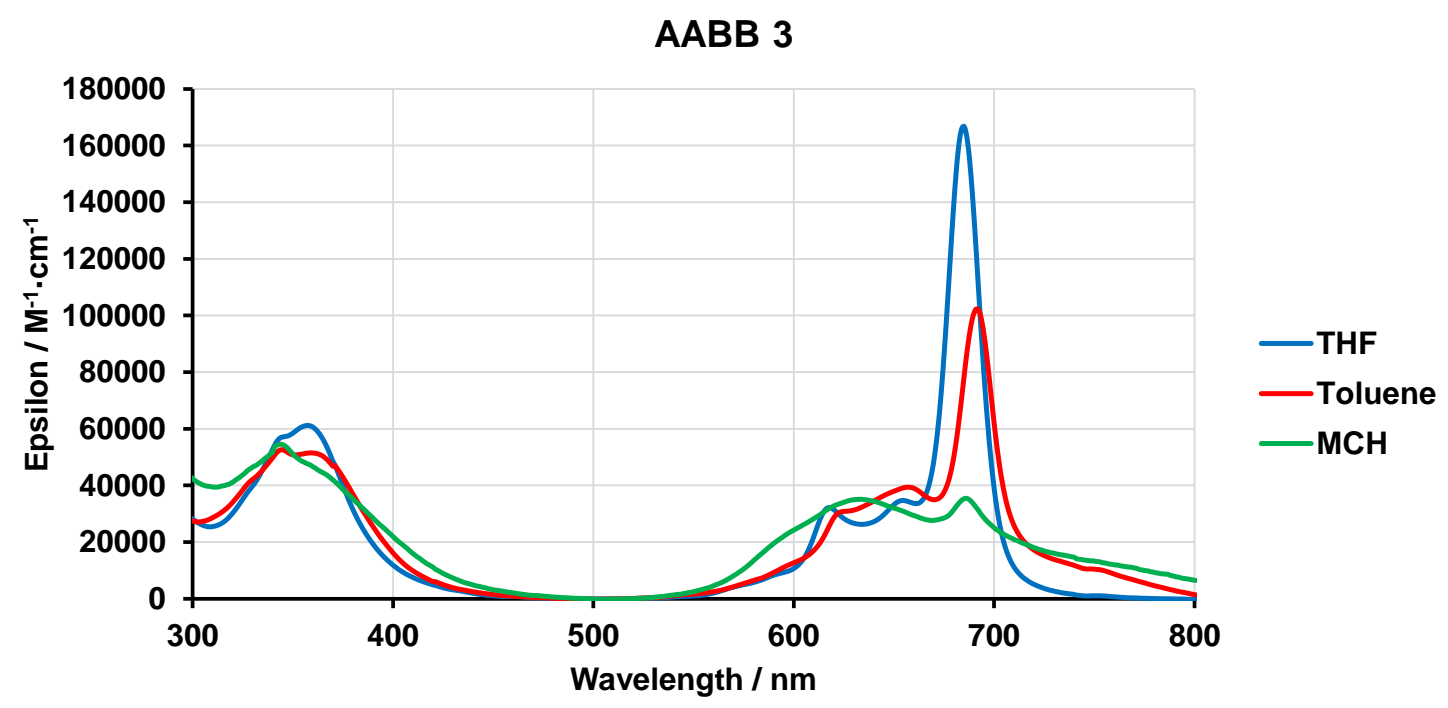

Figure S26. Normalized UV-Vis and Fluorescence spectra for ZnPc 3 in THF and normalized UVVis spectra comparison in THF, toluene and $\mathrm{MCH}$.

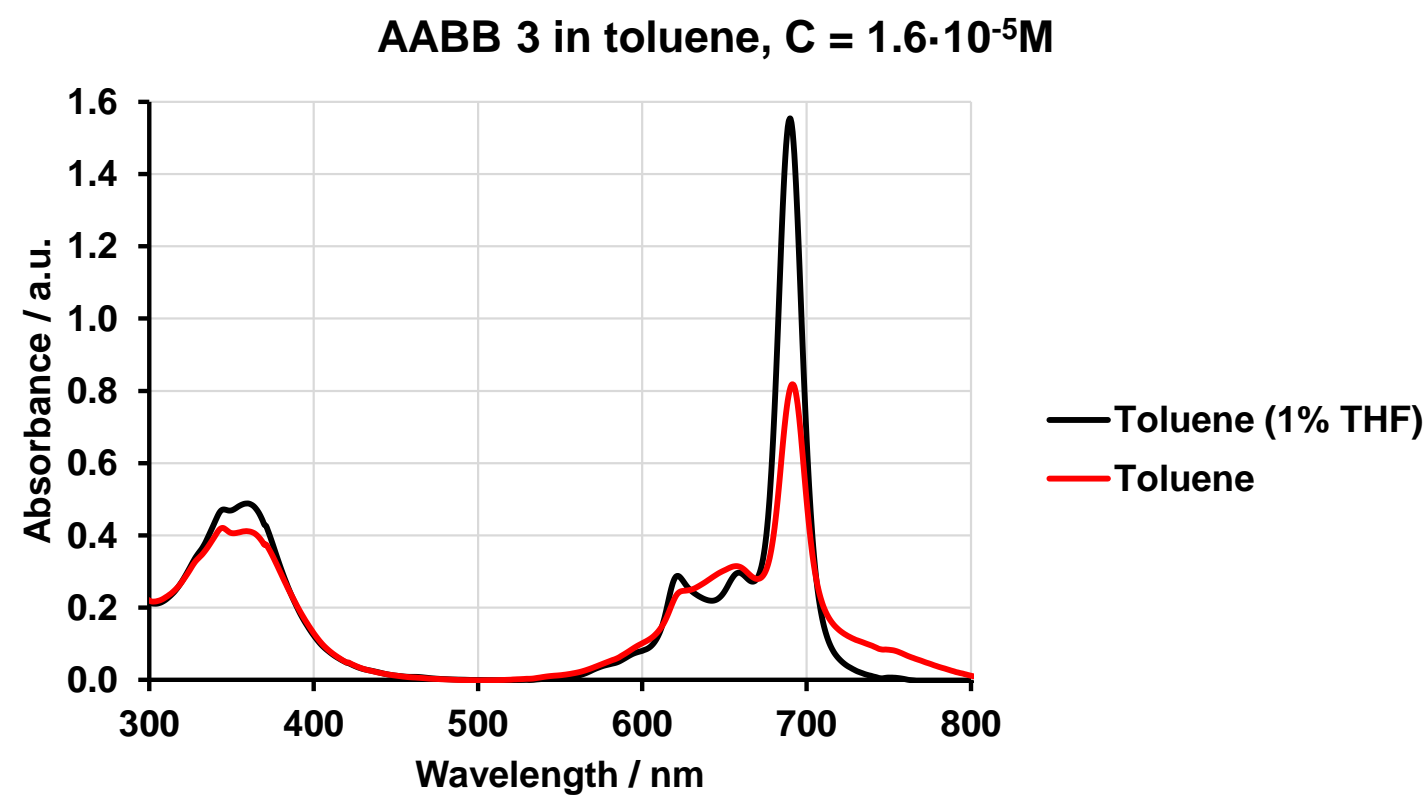




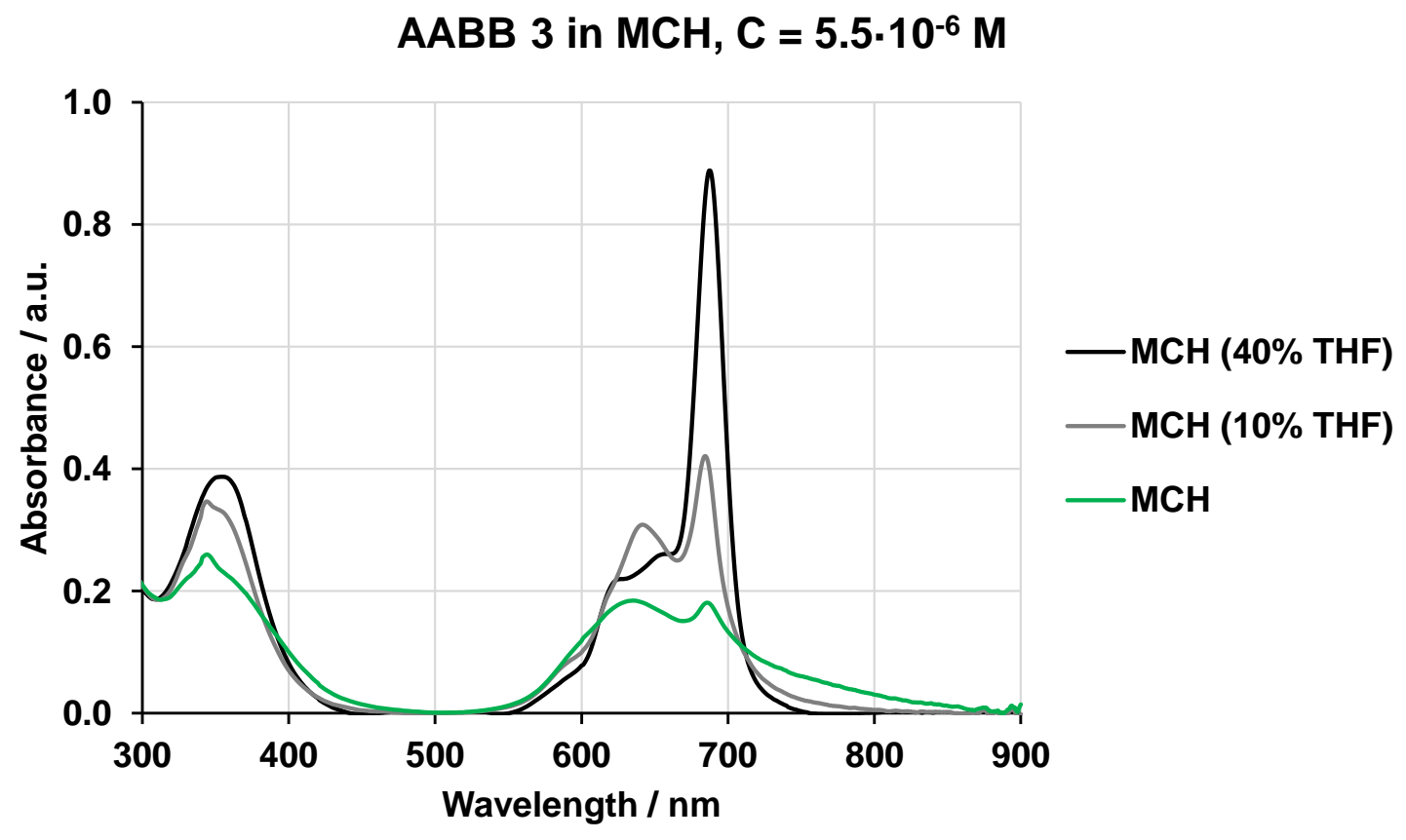

Figure S27. Disaggregation of ZnPc 3 in toluene and MCH upon addition of THF. 

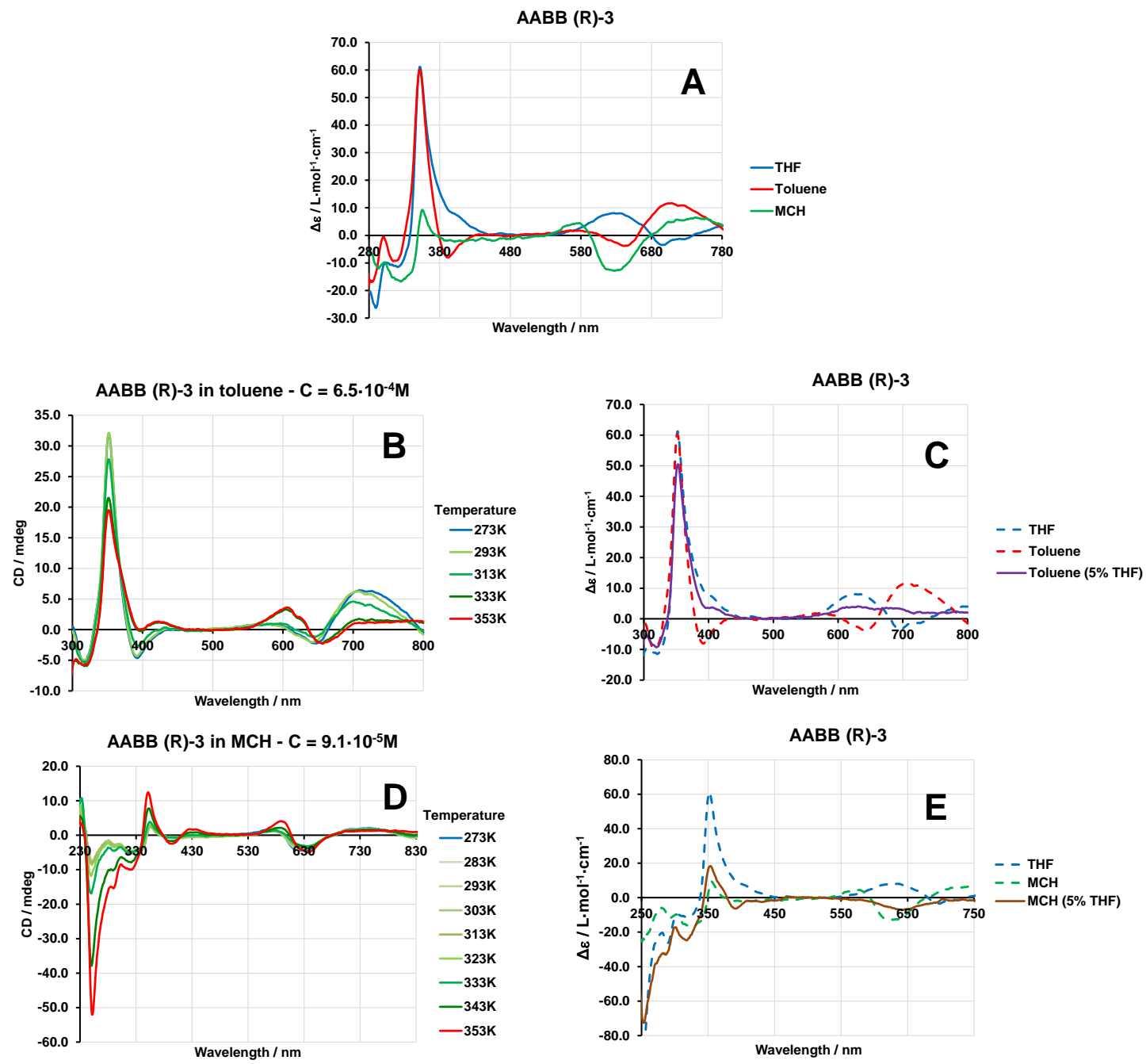

Figure S28. A) Normalized $\mathrm{CD}$ of $\mathrm{ZnPc} 3$ in THF, toluene and $\mathrm{MCH}$. B and D) Temperature dependence in toluene and $\mathrm{MCH}$. $\mathrm{C}$ and $\mathrm{E}$ ) Disaggregation upon addition of THF over toluene and $\mathrm{MCH}$ solutions.

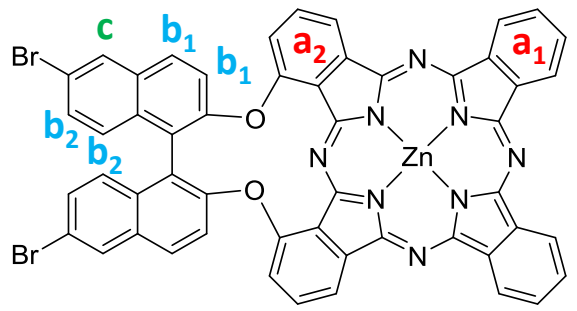

Figure S29. Structure of $\mathrm{ZnPc} 4$ and $\mathrm{H}$ assignation for ${ }^{1} \mathrm{H}-\mathrm{NMR}$. 


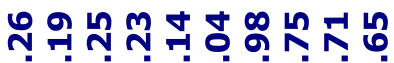

ดั Оं

\section{h} M.M. Mut

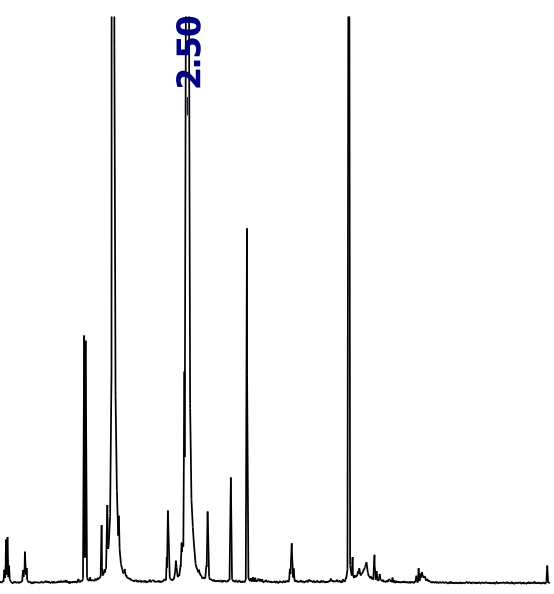

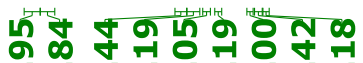
$m$ m

\begin{tabular}{llllllllll}
\hline 9.5 & 8.5 & 7.5 & 6.5 & 5.5 & 4.5 & 3.5 & 2.5 & 1.5 & 0.5
\end{tabular}

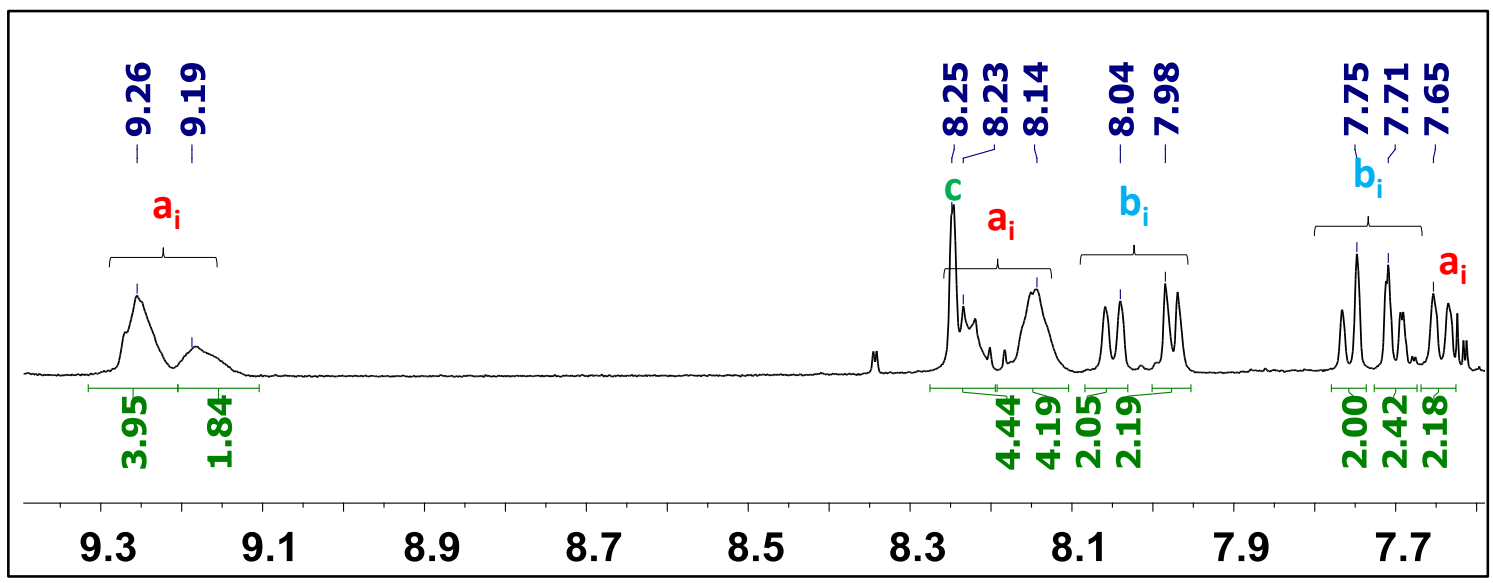

Figure S30. ${ }^{1} \mathrm{H}-\mathrm{NMR}$ of $\mathrm{ZnPc} 4$ in $\mathrm{DMSO}_{-} \mathrm{d}_{6}$, at $363 \mathrm{~K}$ and magnification from 7.6 to $9.3 \mathrm{ppm}$. 


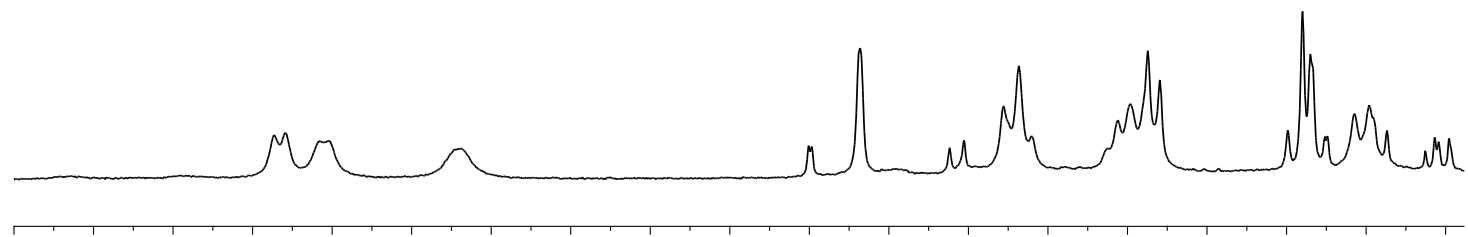
9.3
9.1
8.9
8.7
8.5
8.3
8.1
7.9
7.7

AABB 4, DMSO-d 6 , 500MHz, 363K

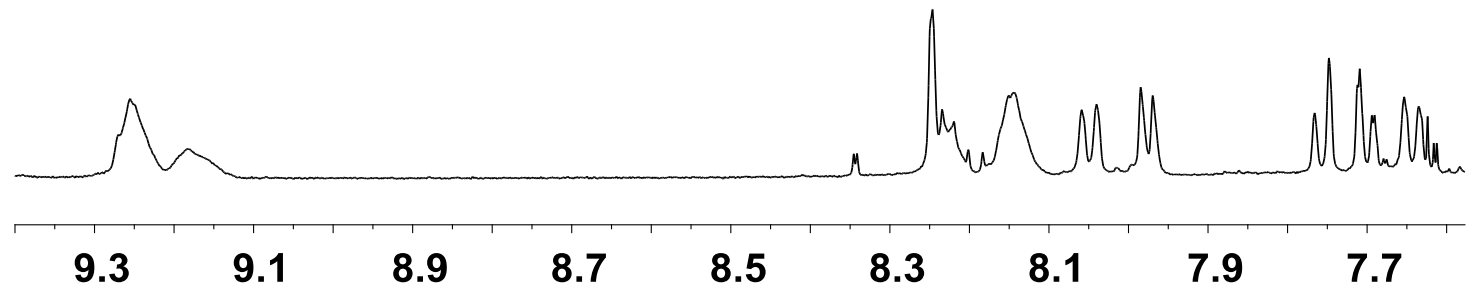

Figure S31. ${ }^{1} \mathrm{H}-\mathrm{NMR}$ variation with temperature, 298K (top) and 363K (down), of ZnPc 4 in DMSO- $d_{6}$.

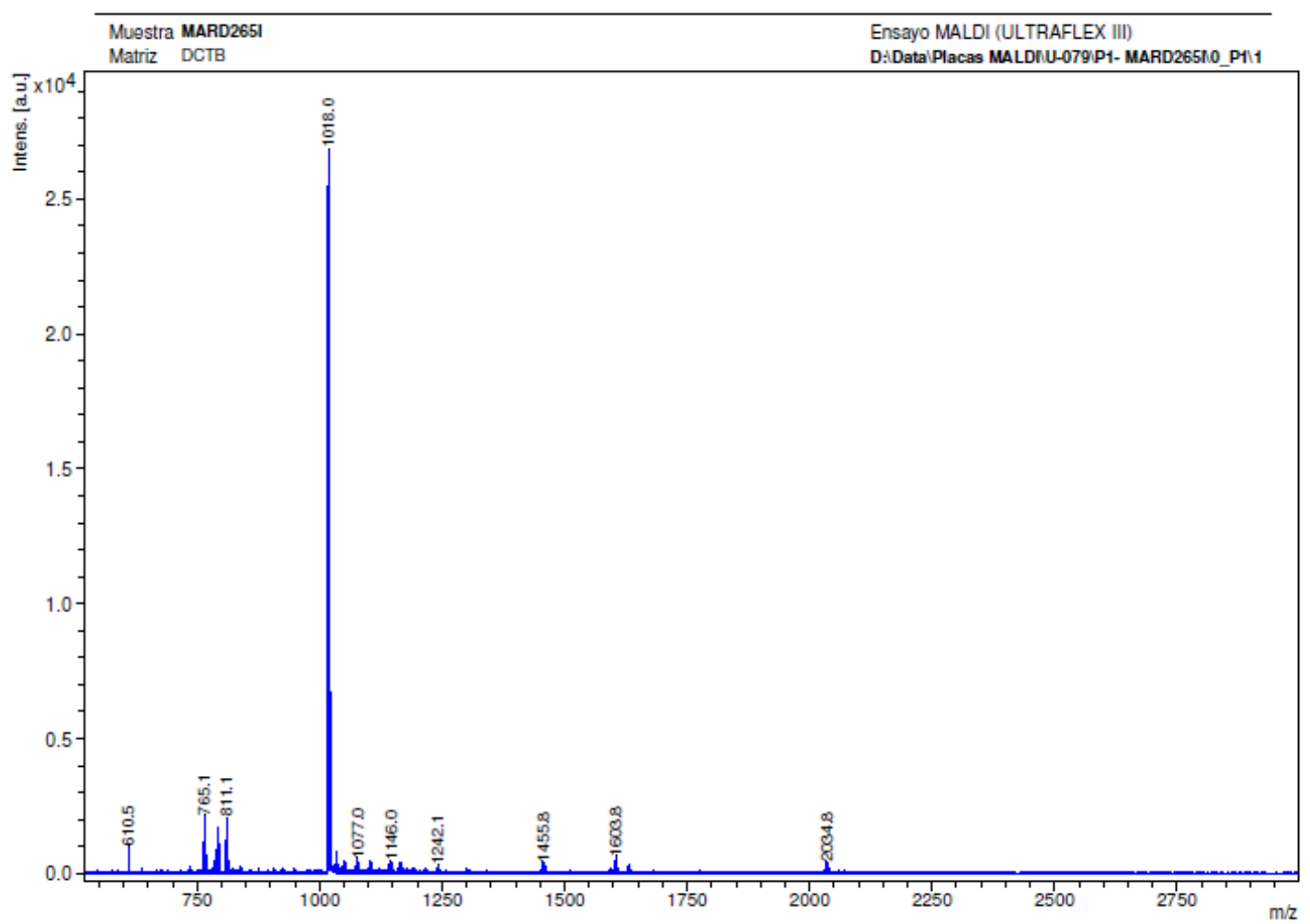




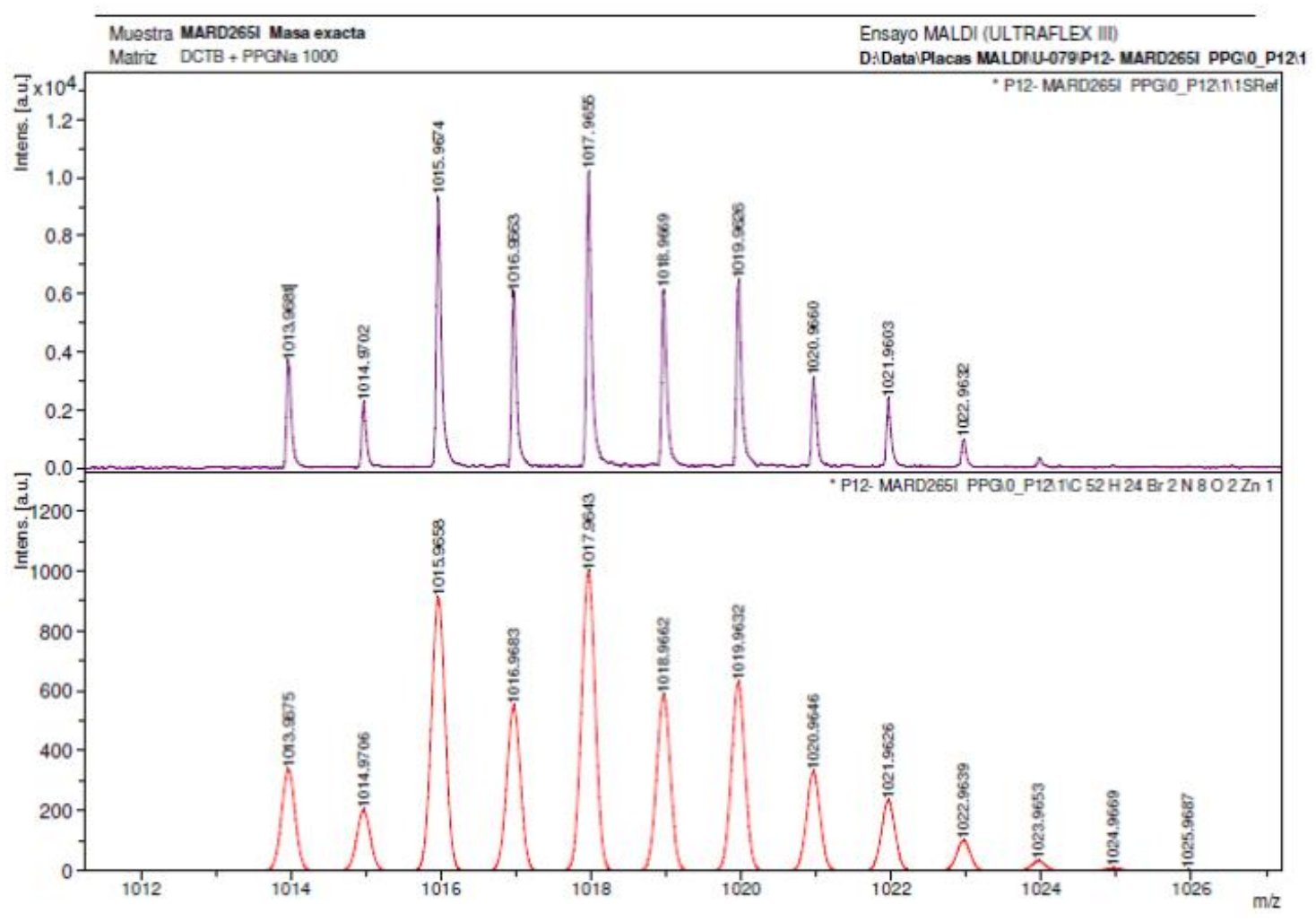

Figure S32. MS and HR-MS of ZnPc 4.

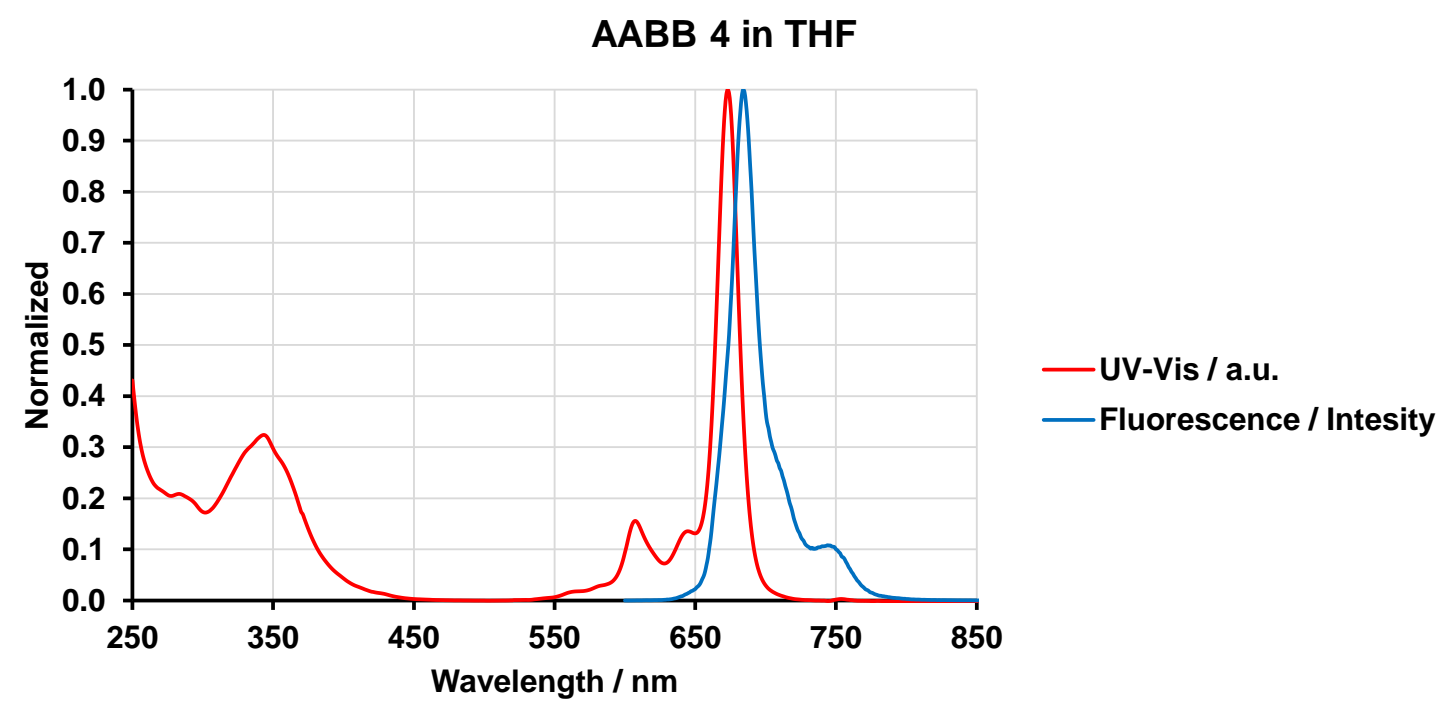

Figure S33. Normalized UV-Vis and Fluorescence spectra for ZnPc 4 in THF. 


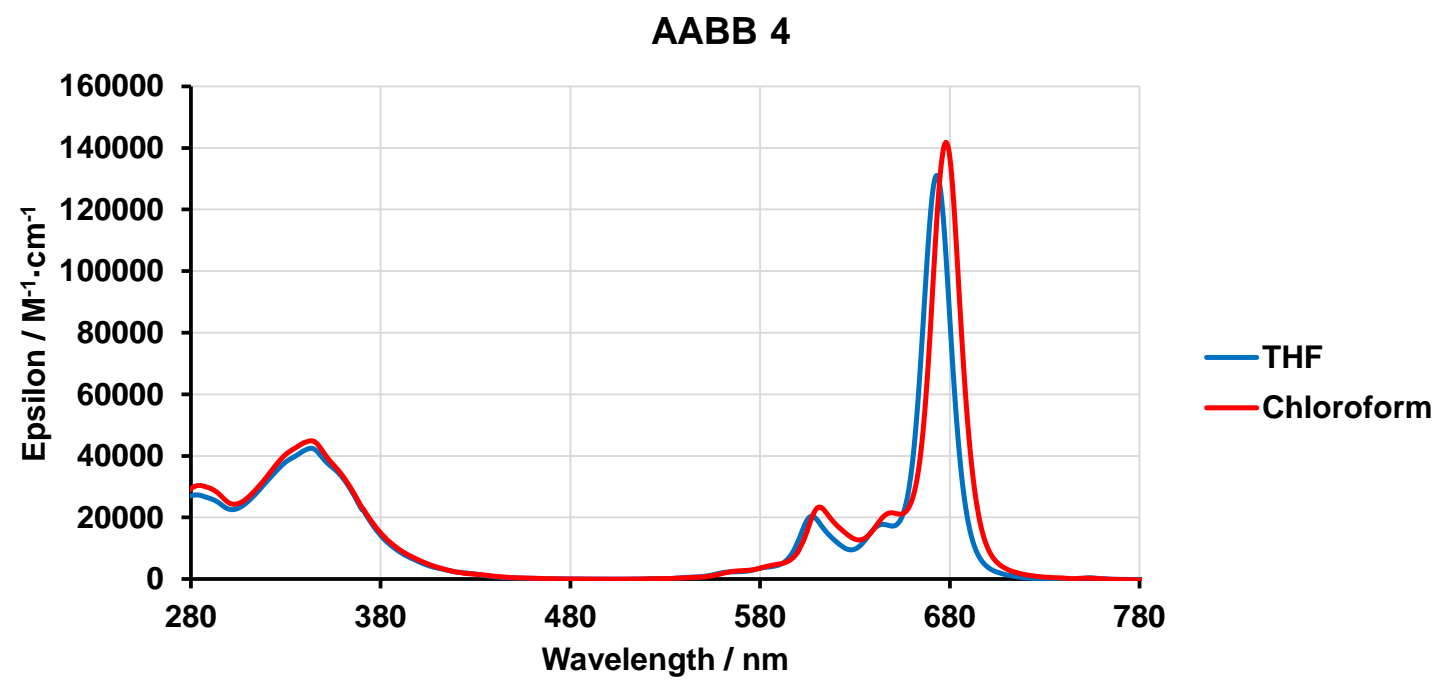

Figure S34. Normalized UV-Vis spectrum of $\mathrm{ZnPc} 4$ in $\mathrm{THF}$ and $\mathrm{CHCl}_{3}$ as non-coordinative solvent ( $\mathrm{ZnPc} \mathbf{4}$ is insoluble in toluene). Aggregation phenomena are not clearly observed.

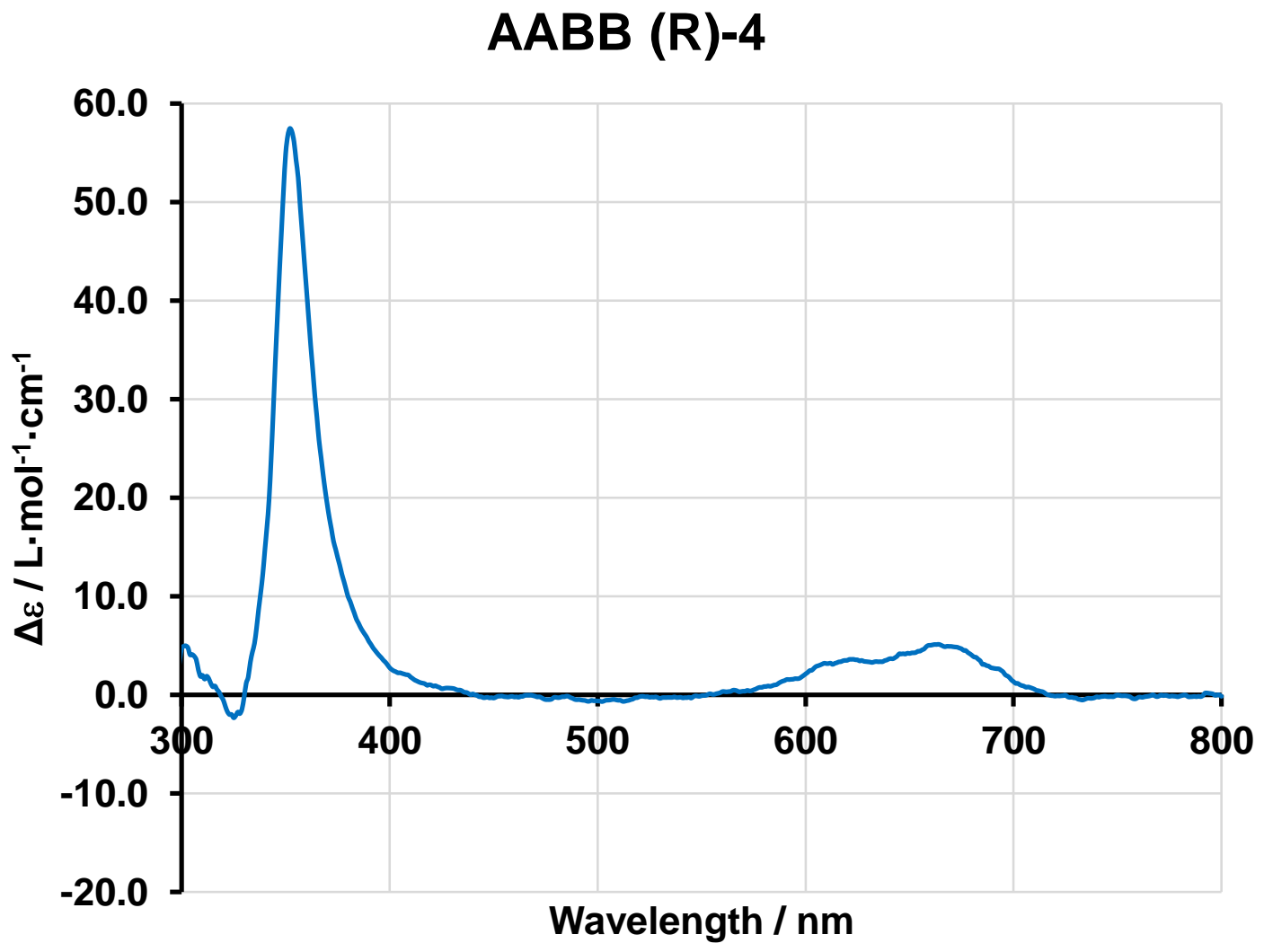

Figure S35. Normalized CD of ZnPc 4 in THF. 


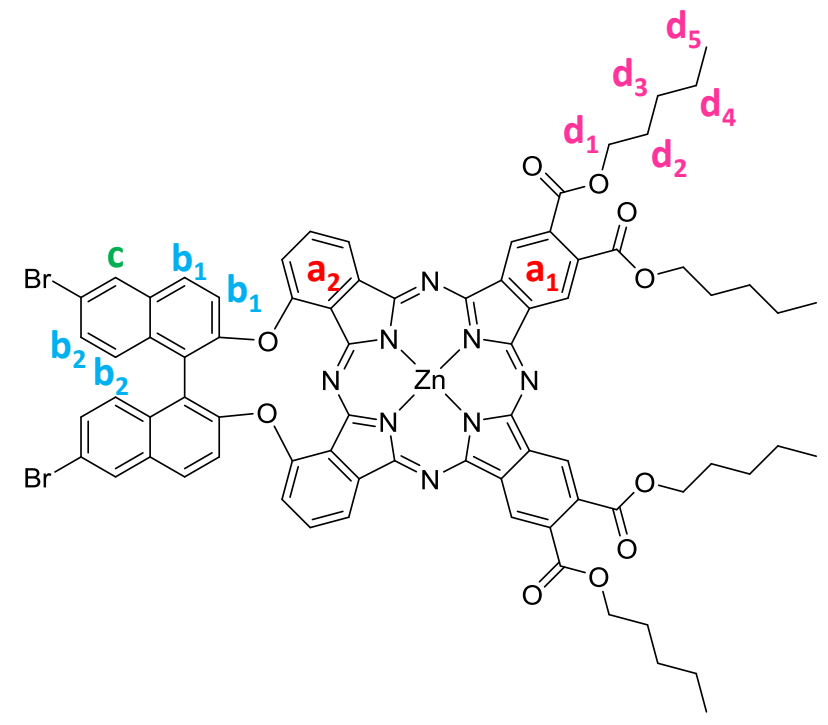

Figure S36. Structure of ZnPc 5 and $\mathrm{H}$ assignation for ${ }^{1} \mathrm{H}-\mathrm{NMR}$.

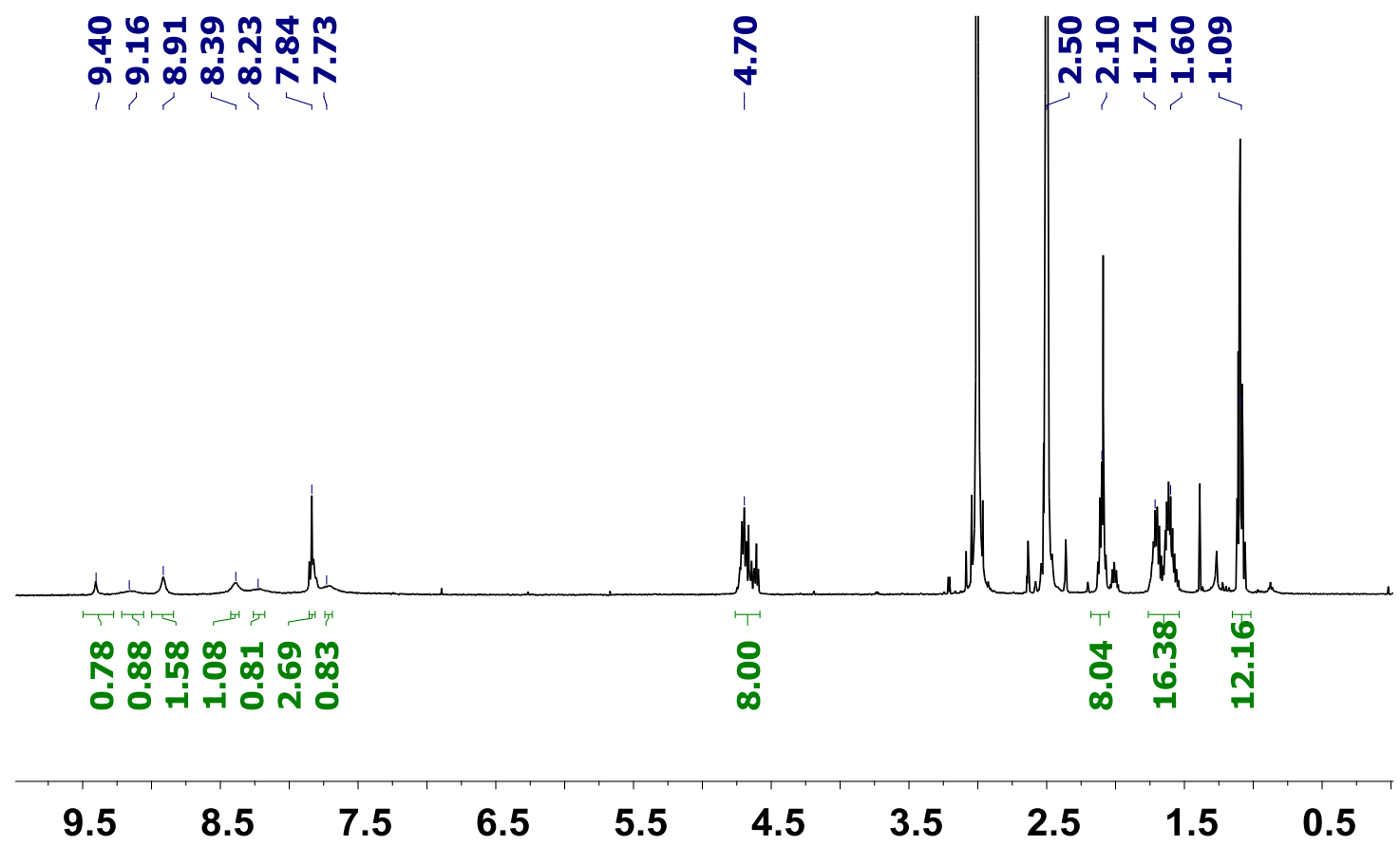



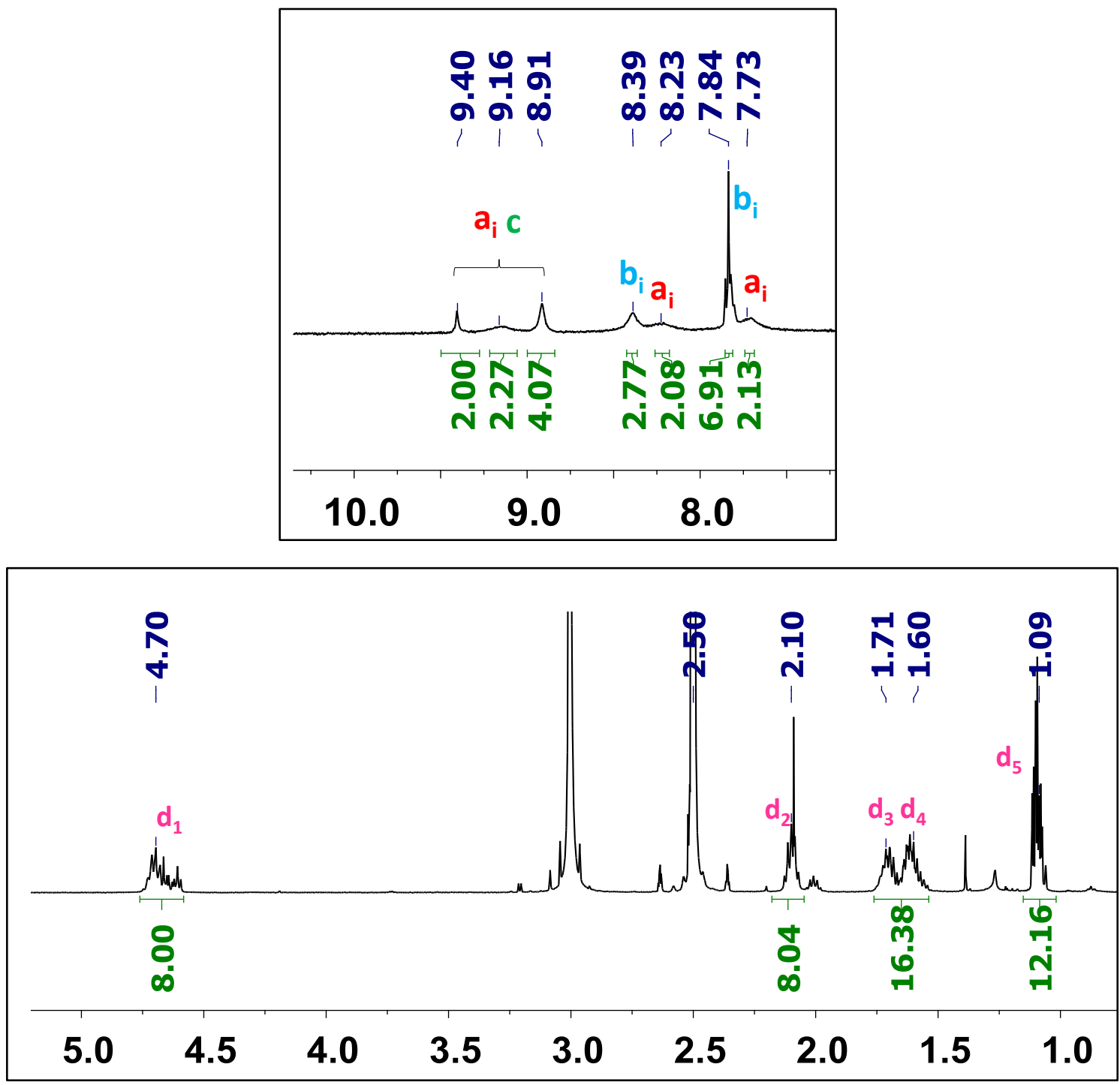

Figure S37. ${ }^{1} \mathrm{H}-\mathrm{NMR}$ of $\mathrm{ZnPc} 5$ in DMSO-d $\mathrm{d}_{6}$, at 363K. As the product present aggregation in the aromatic region integrals for de alkyl chains were calculated independently just to check the relation between the protons of alkyl chains fits properly. And magnification from 1 to 5 ppm and from 7.5 to 10 ppm. 

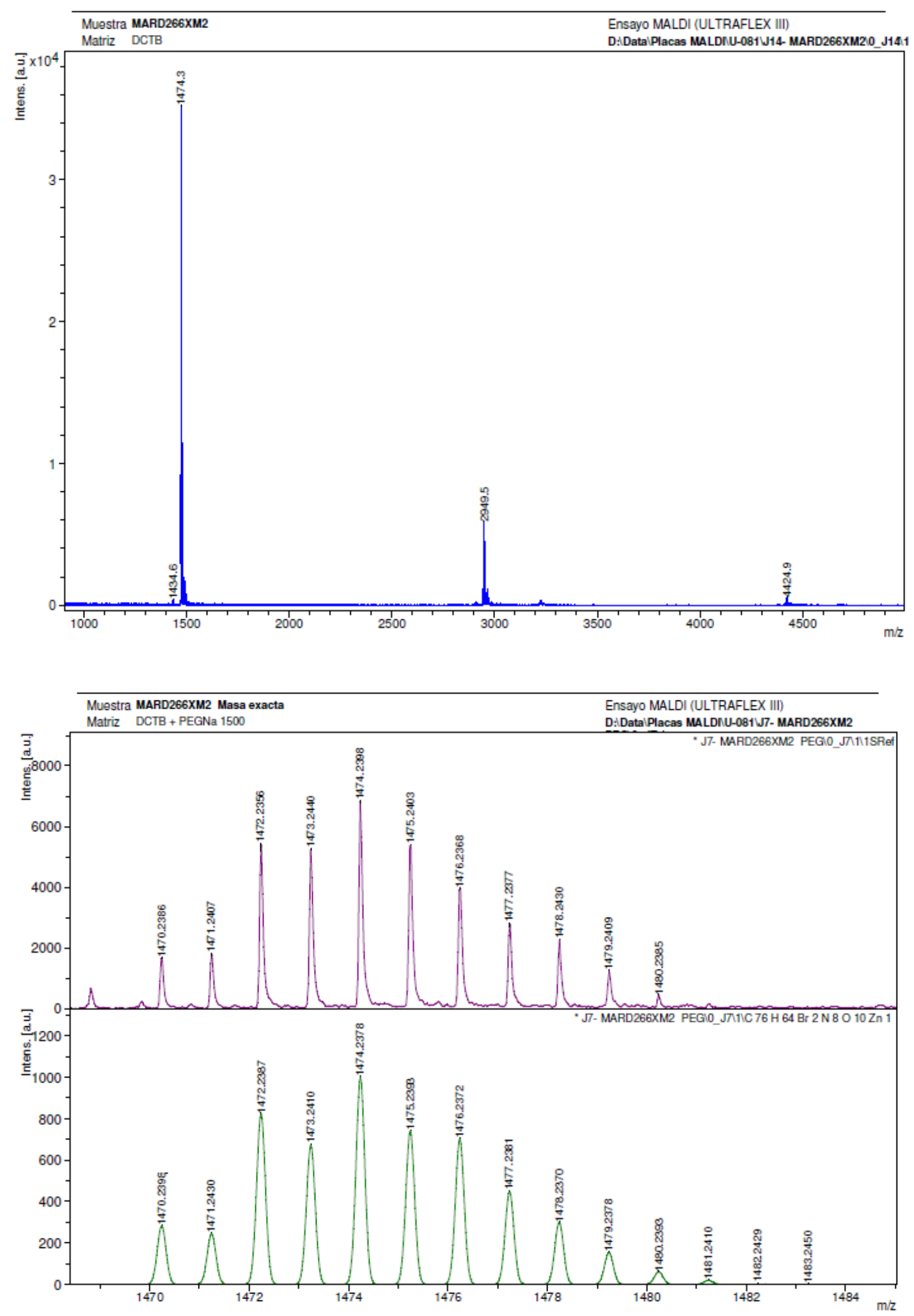

Figure S38. MS and HR-MS of ZnPc 5. 


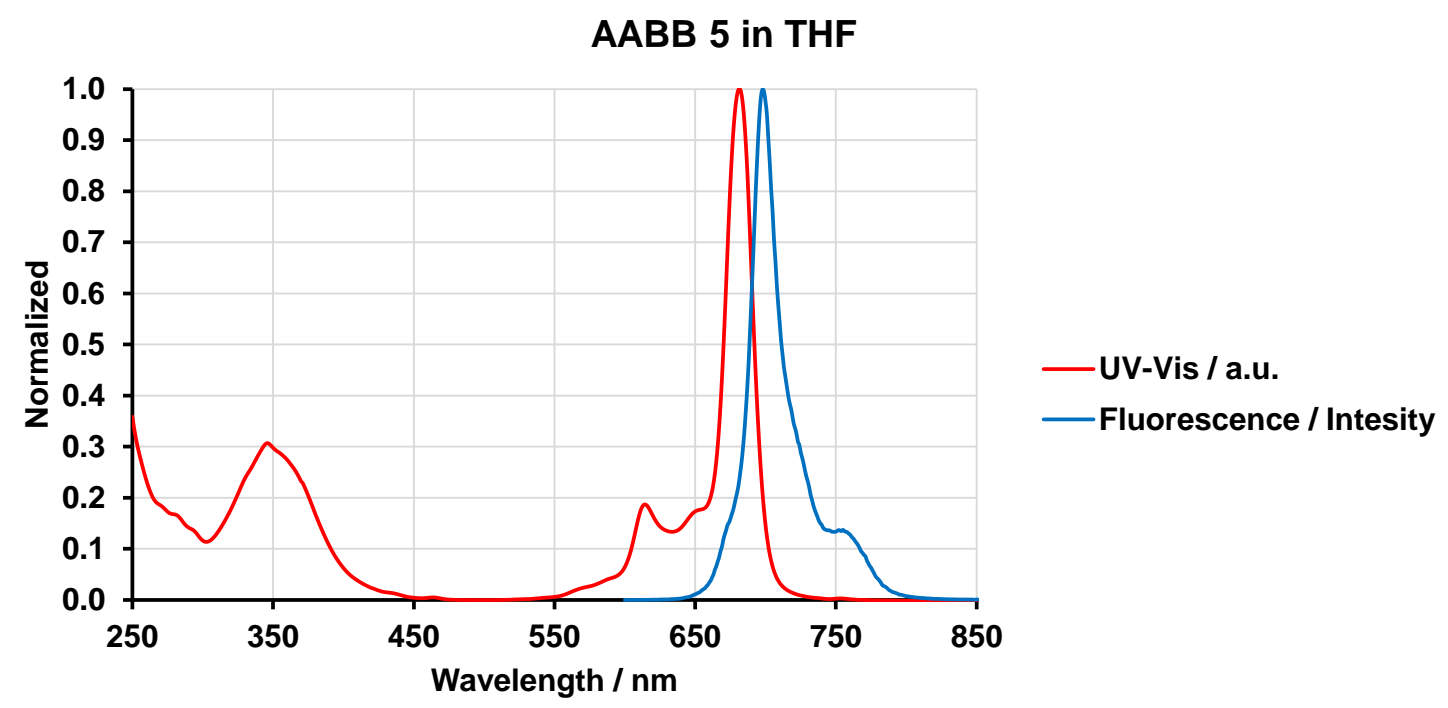

Figure S39. Normalized UV-Vis and Fluorescence spectra for ZnPc 5 in THF.

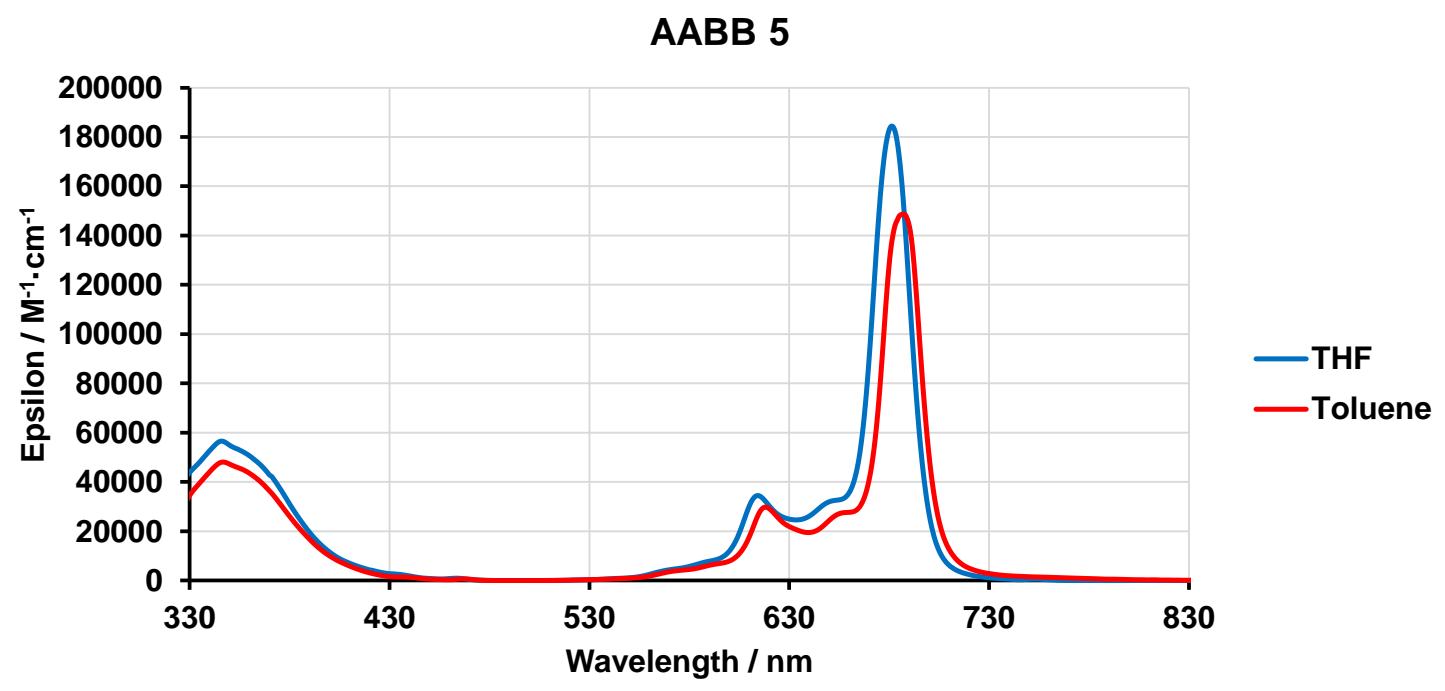

Figure S40. Normalized UV-Vis spectrum of ZnPc 5 in THF and toluene as non-coordinative solvent. Aggregation phenomena are not clearly observed. 


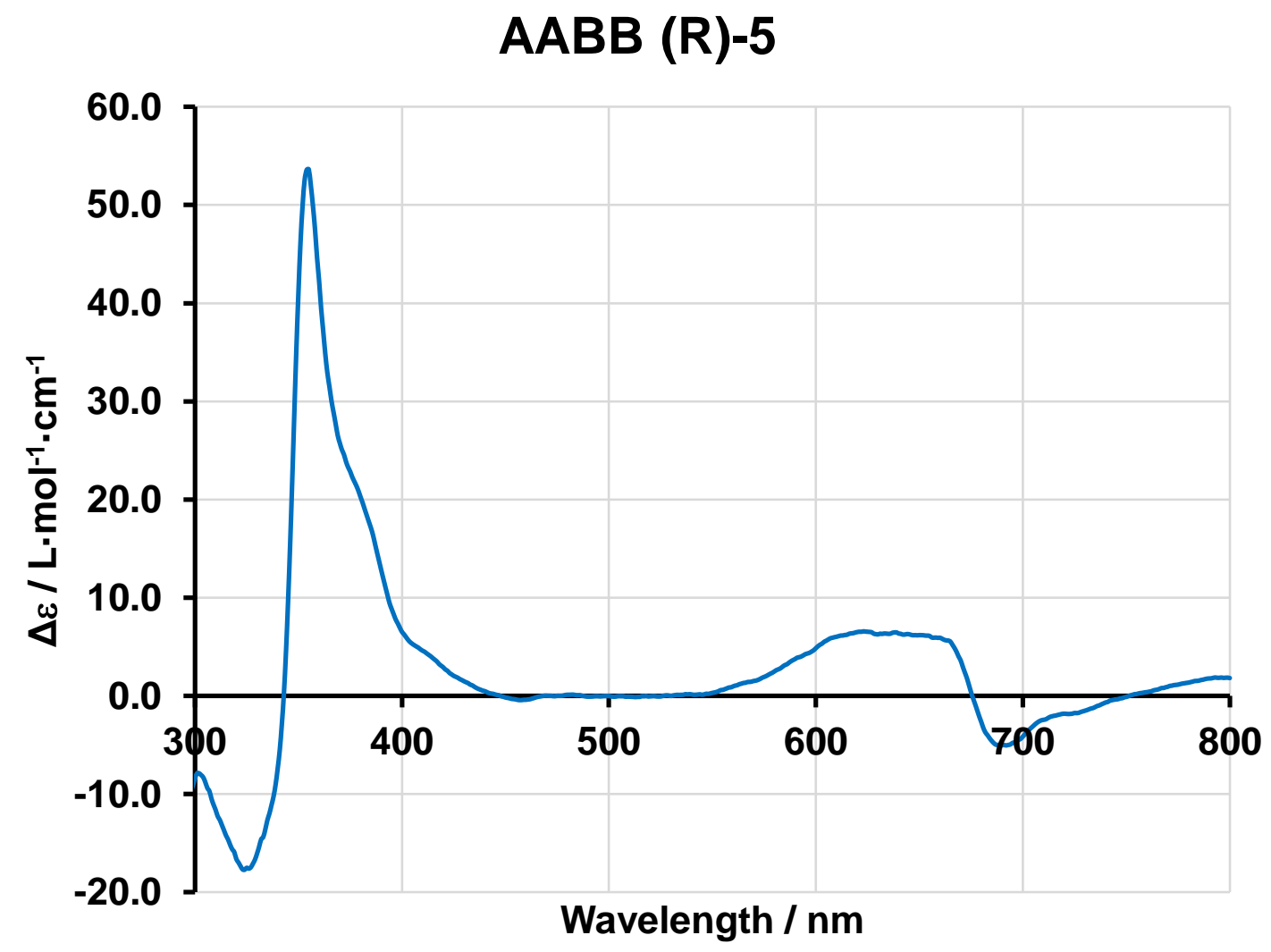

Figure S41. Normalized CD in THF of ZnPc 5.

ZnPc 6

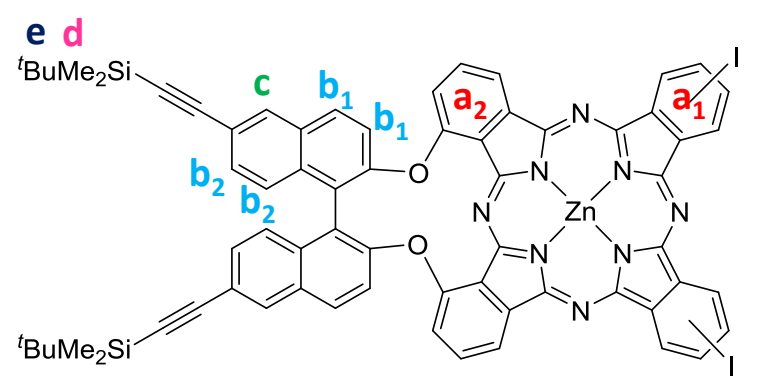

Figure S42. Structure of $\mathrm{ZnPc} 6$ and $\mathrm{H}$ assignation for ${ }^{1} \mathrm{H}-\mathrm{NMR}$. 


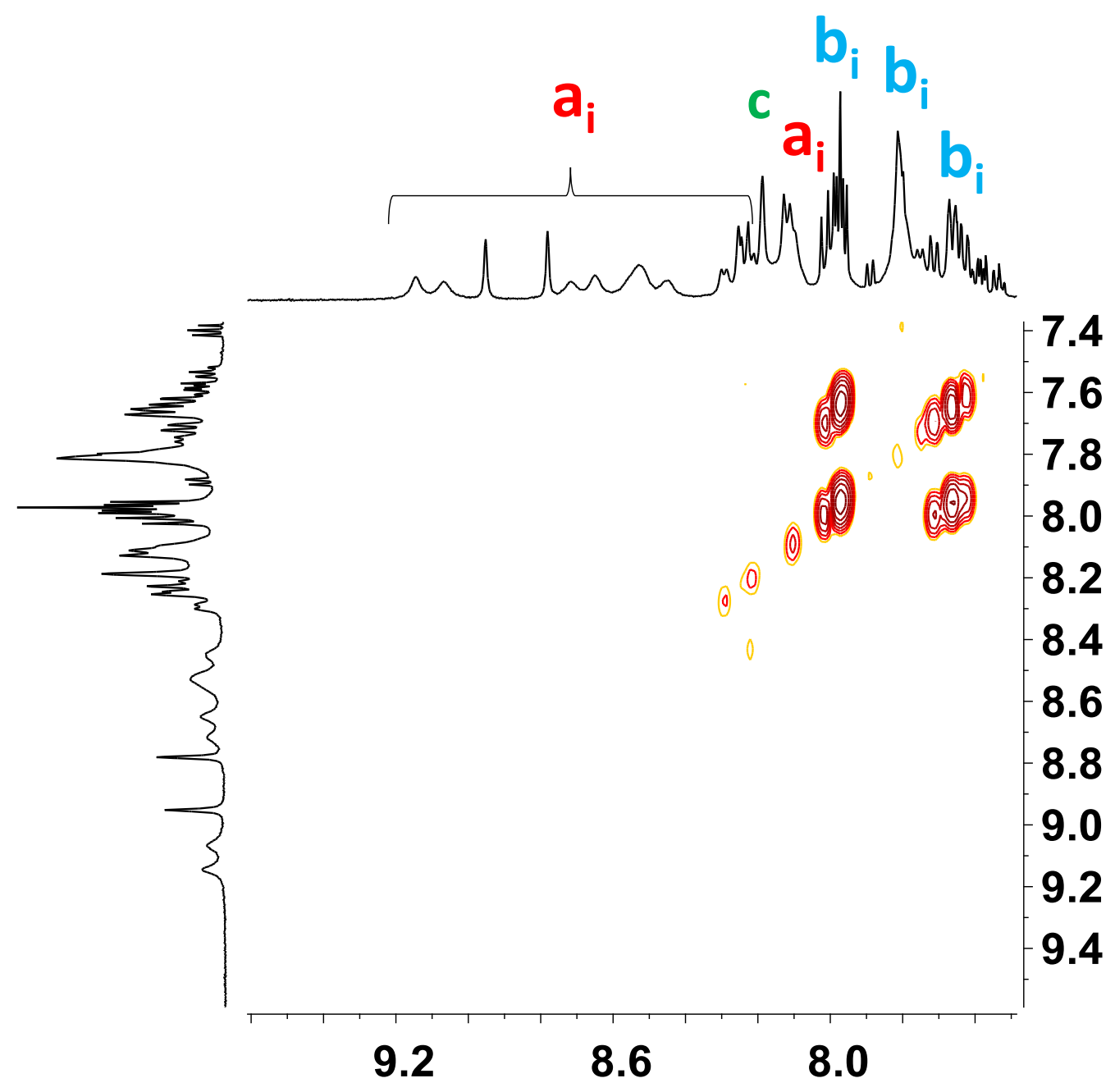



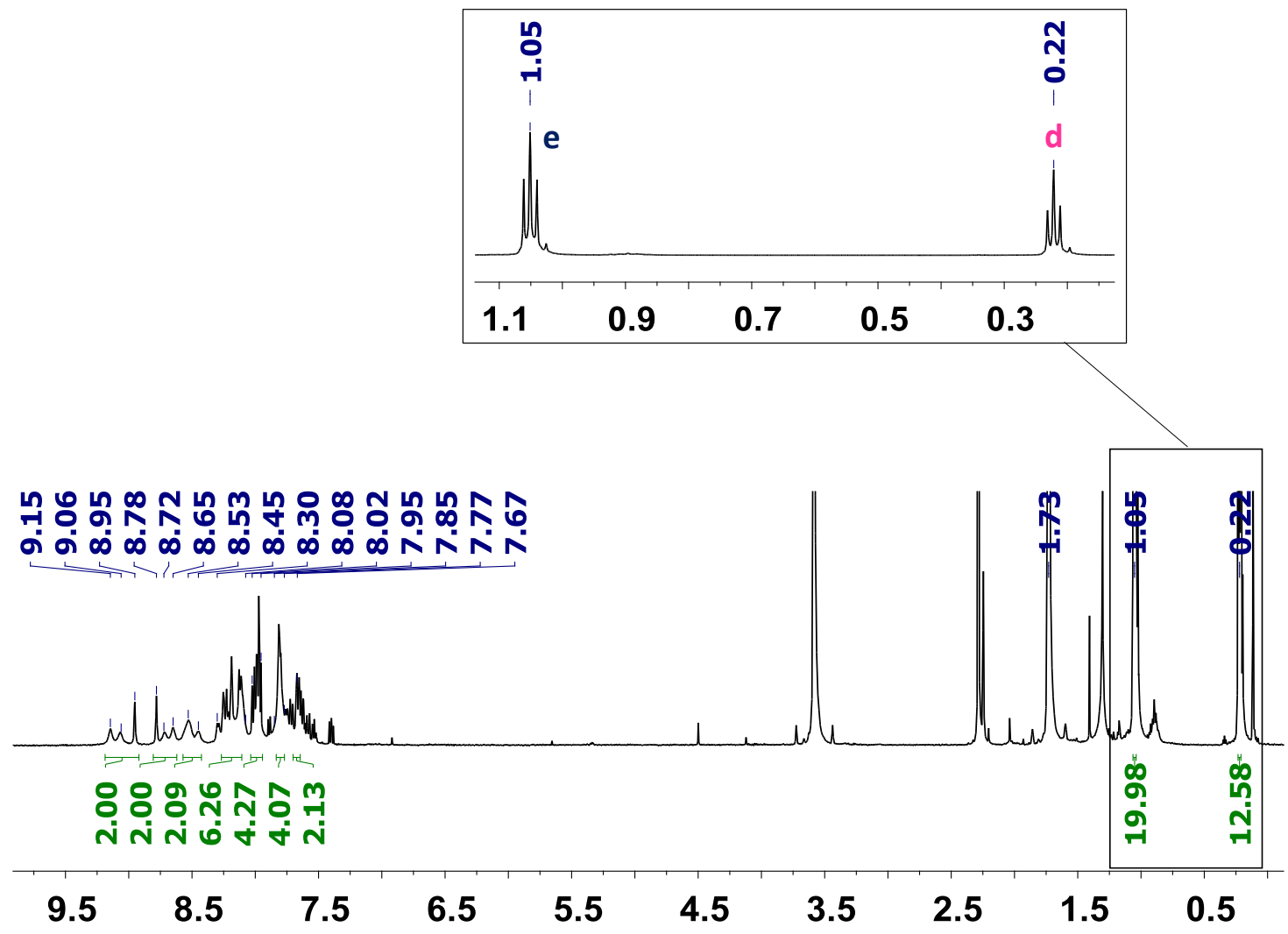

Figure S43. ${ }^{1} \mathrm{H}-\mathrm{NMR}$ and COSY of ZnPc 6 in THF- $\mathrm{d}_{8}$, at $323 \mathrm{~K}$. 
AABB 6, THF-d8, 500MHz, 298K

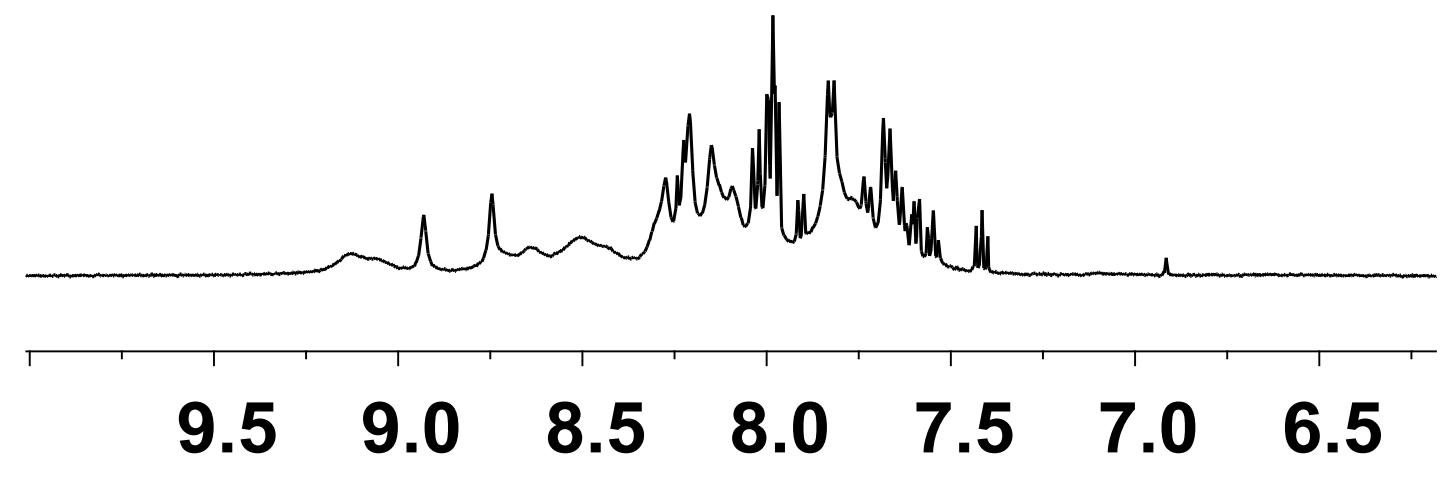

AABB 6, THF-d8, 500MHz, 323K

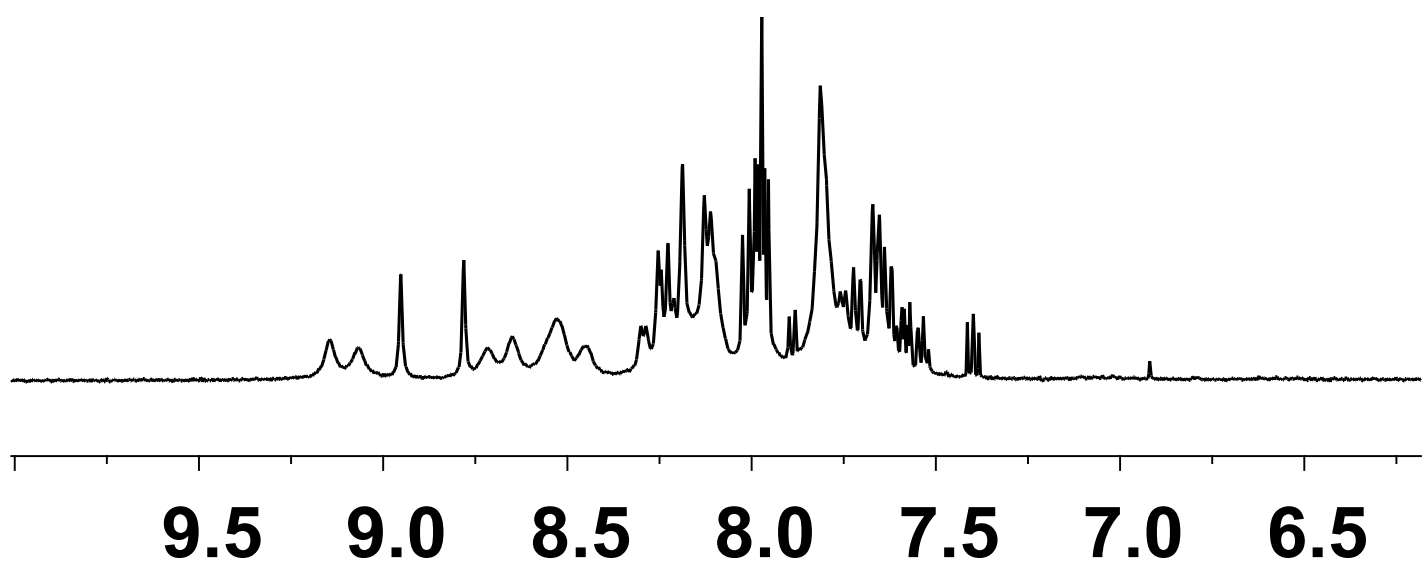

Figure S44. ${ }^{1} \mathrm{H}-\mathrm{NMR}$ variation with temperature, $298 \mathrm{~K}$ (top) and 323K (down), of ZnPc 6 in THF$d_{8}$. 

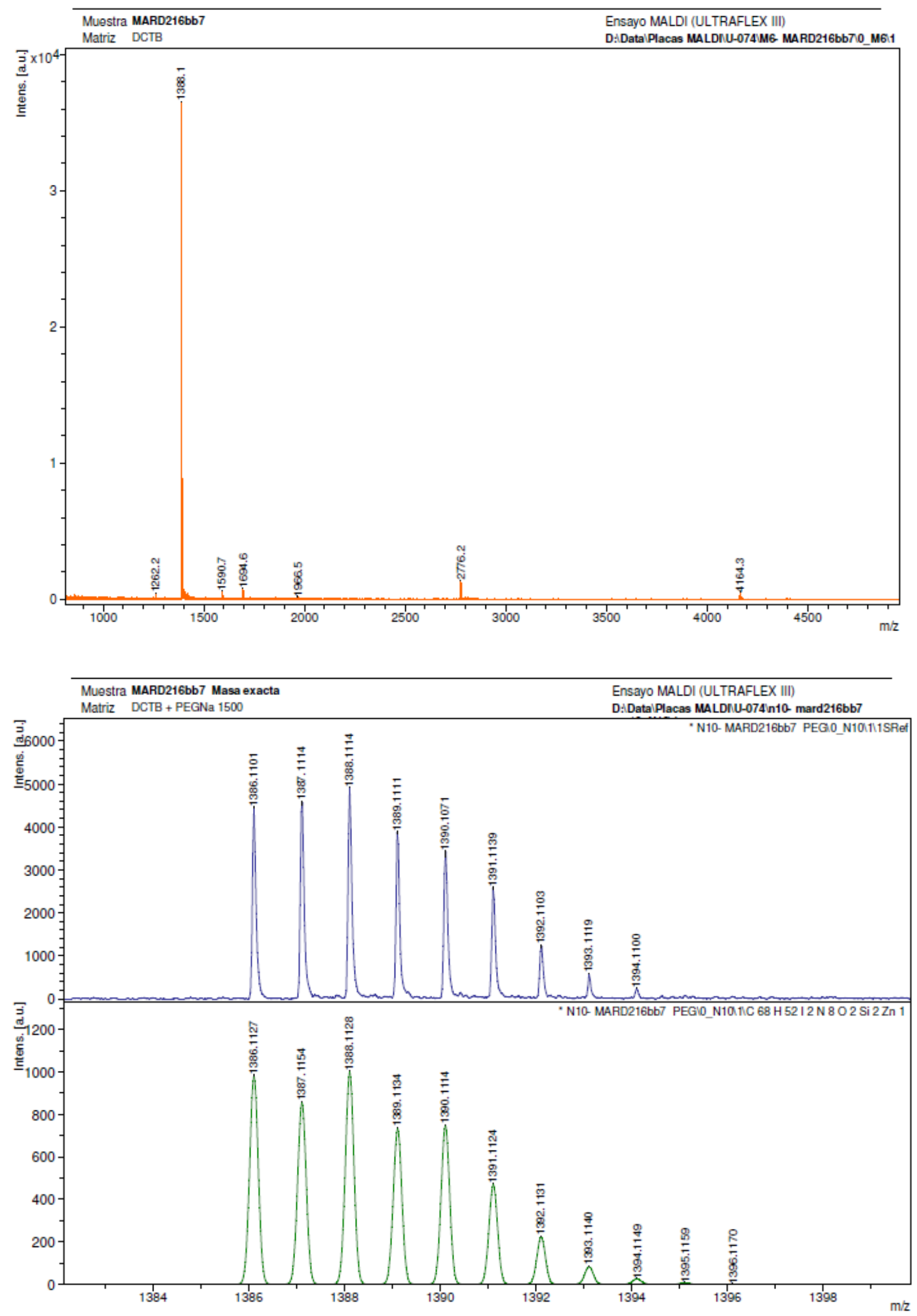

Figure S45. MS and HR-MS of ZnPc 6. 

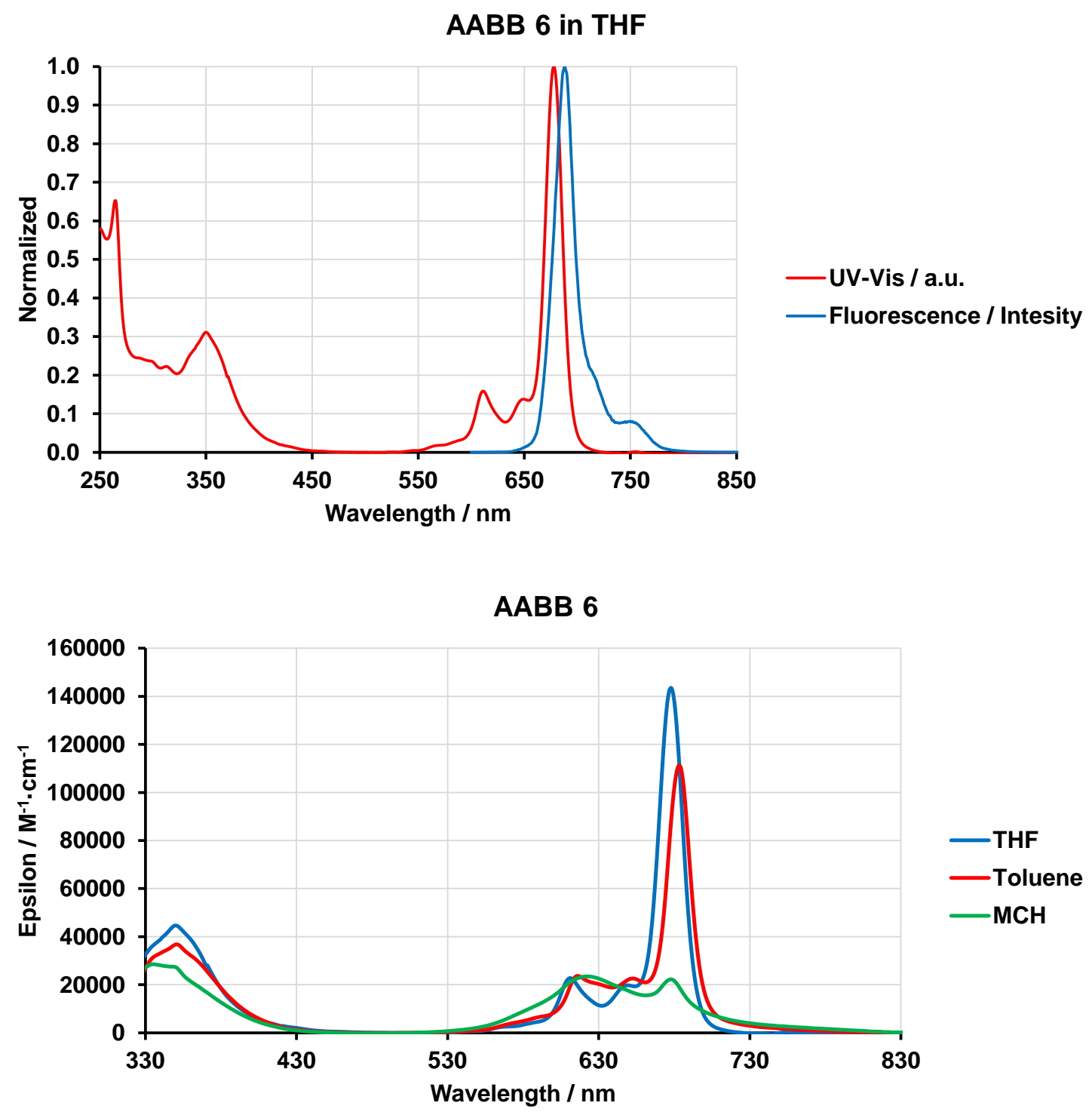

Figure S46. Normalized UV-Vis and Fluorescence spectra for ZnPc 6 in THF and normalized UVVis spectra comparison in THF, toluene and $\mathrm{MCH}$. 

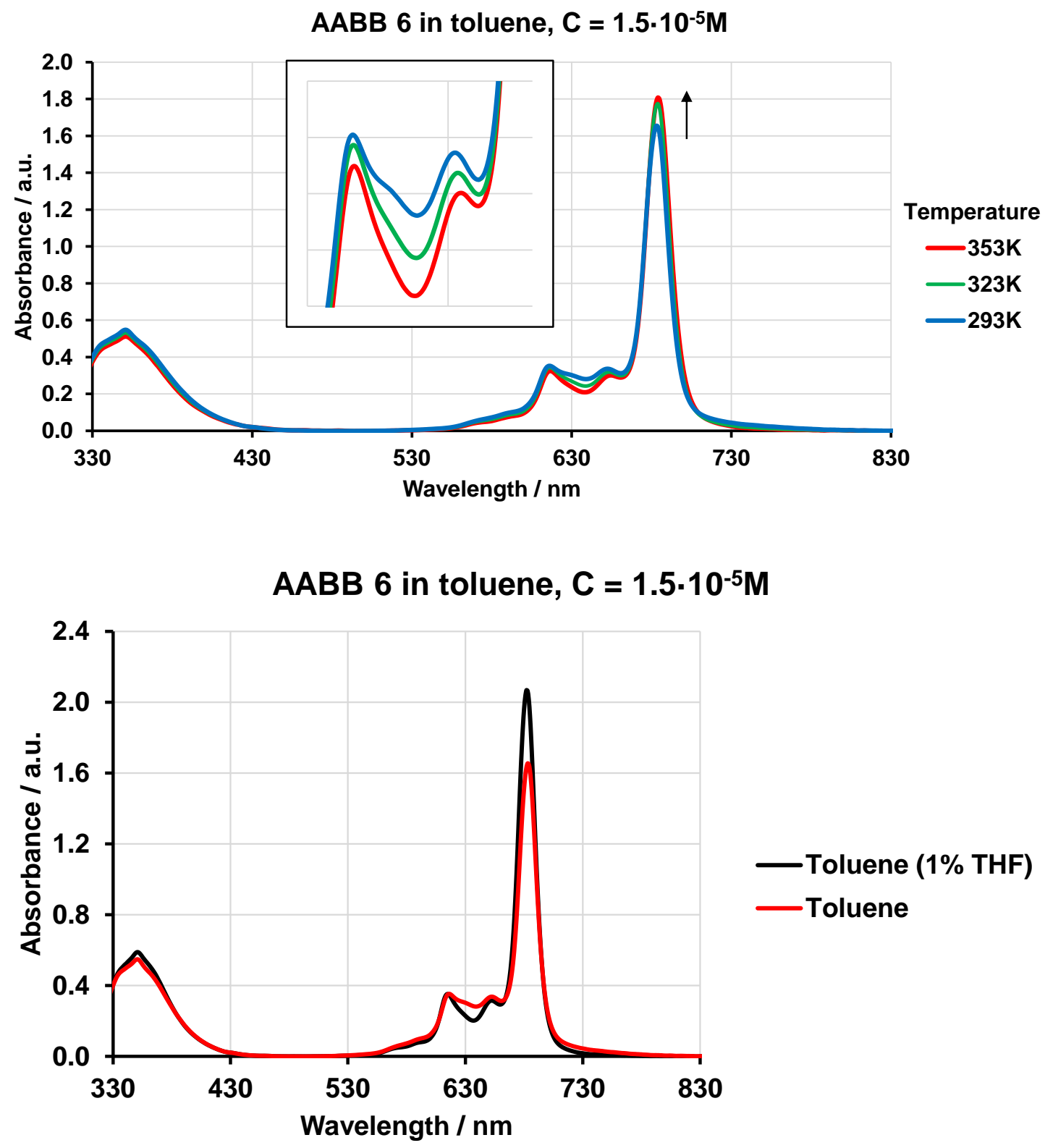

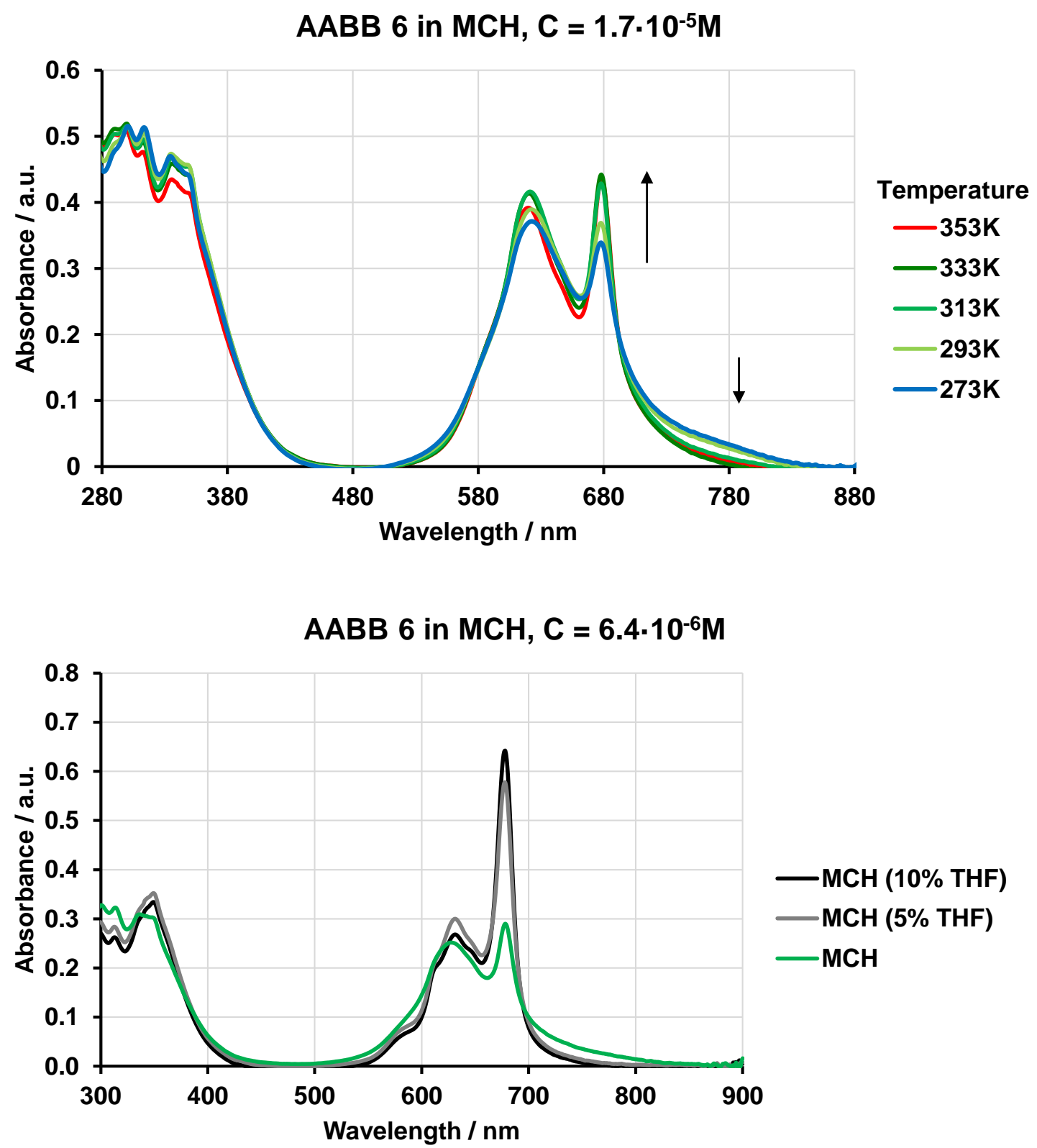

Figure S47. Disaggregation of $\mathrm{ZnPc} 6$ in toluene and $\mathrm{MCH}$ with temperature and upon addition of THF. 

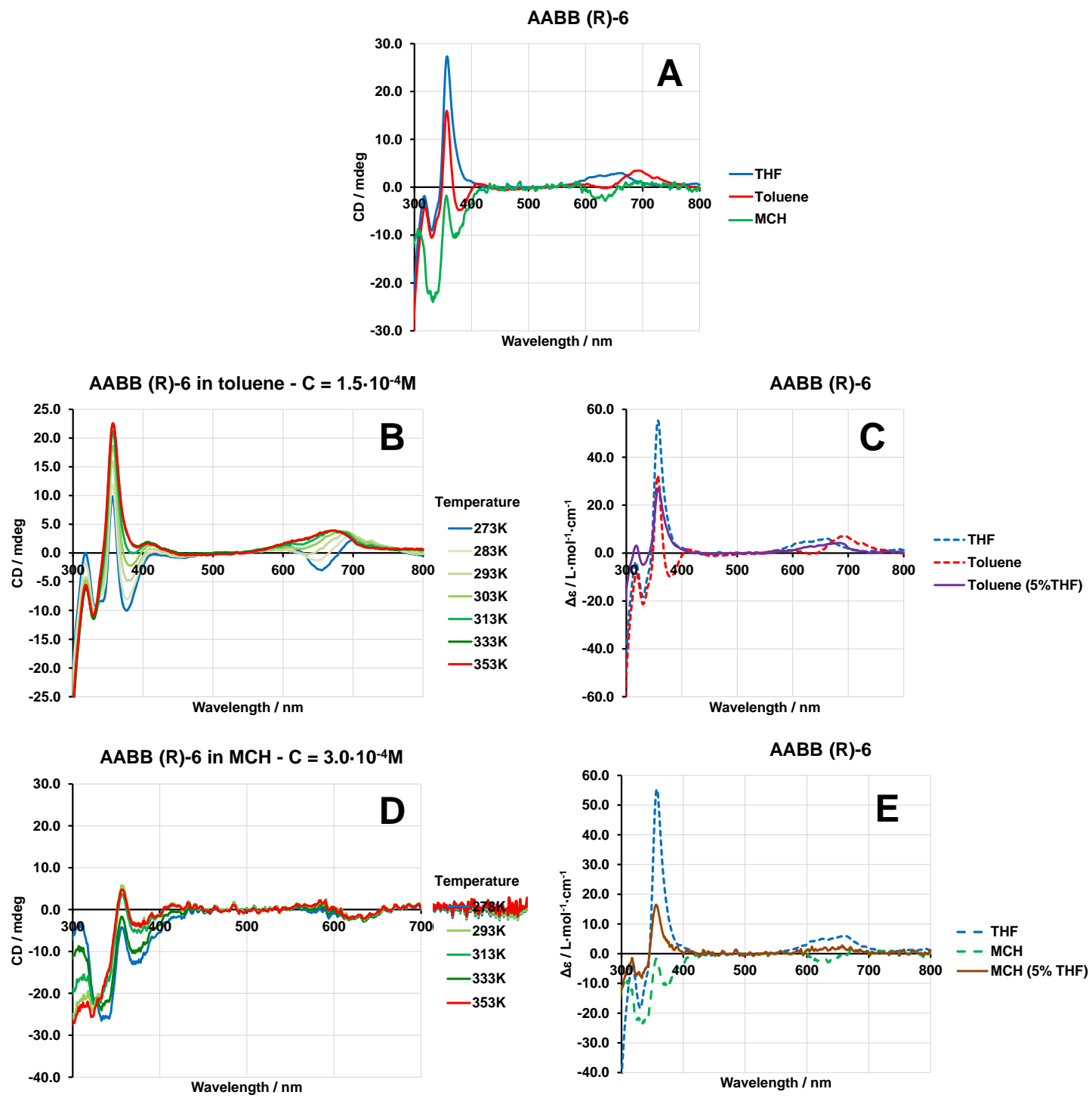

Figure S48. A) Normalized CD of ZnPc 6 in THF, toluene and $\mathrm{MCH}$. B and D) Temperature dependence in toluene and $\mathrm{MCH}$. $\mathrm{C}$ and $\mathrm{E}$ ) Disaggregation upon addition of THF over toluene and $\mathrm{MCH}$ solutions.

$(\mathrm{AA})_{2}-\mathrm{C}$

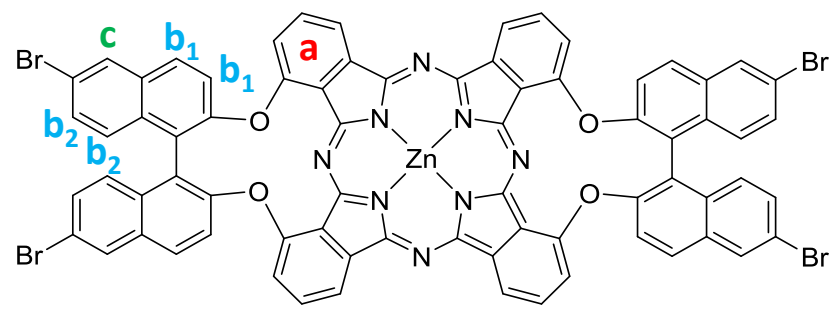

Figure S49. Structure of $(A A)_{2}-\mathrm{C}$ and $\mathrm{H}$ assignation for ${ }^{1} \mathrm{H}-\mathrm{NMR}$. 


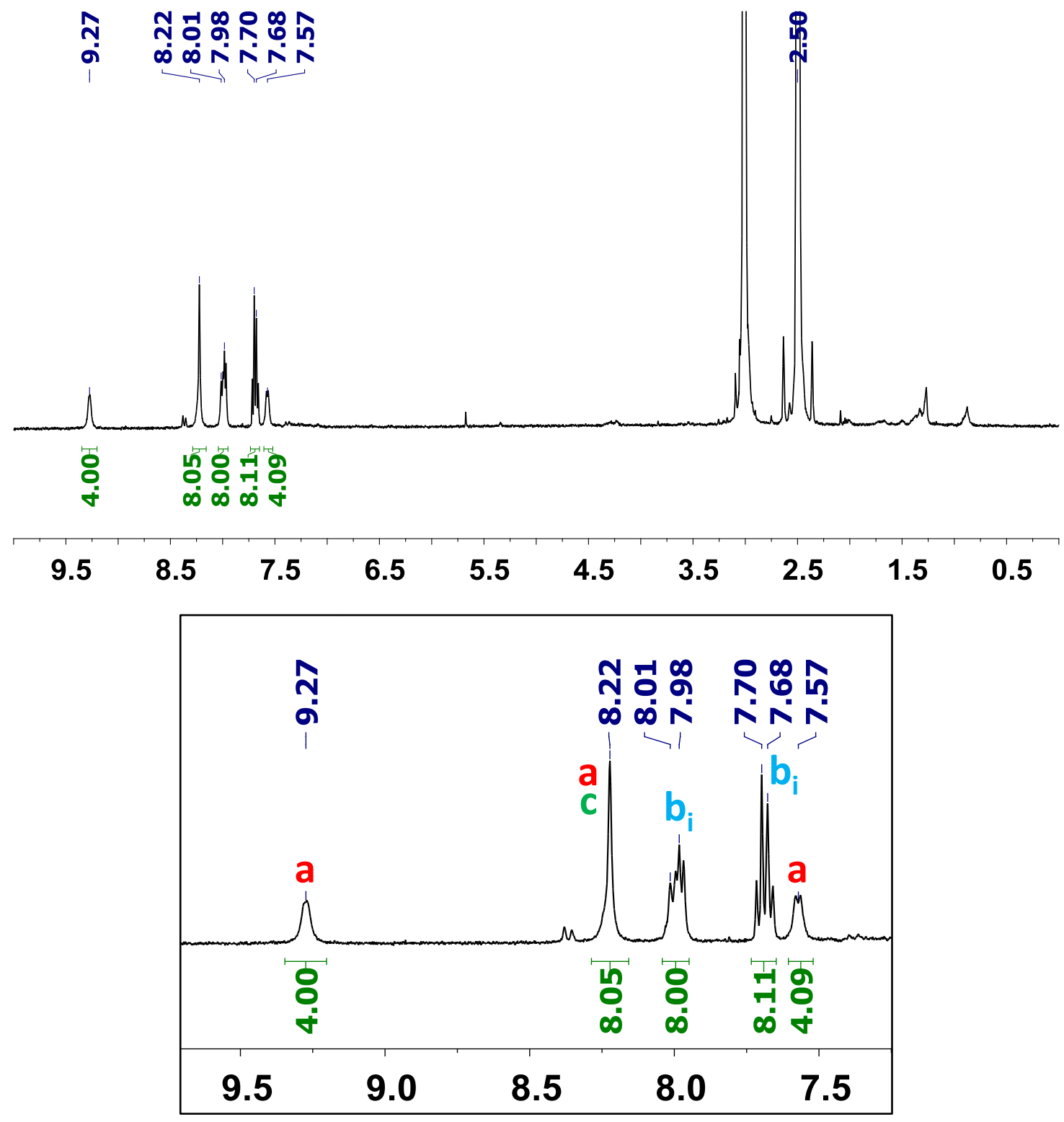

Figure S50. ${ }^{1} \mathrm{H}-\mathrm{NMR}$ of $(\mathrm{AA})_{2}-\mathrm{c}$ in DMSO- $\mathrm{d}_{6}$, at $363 \mathrm{~K}$ and magnification from 7.5 to $9.5 \mathrm{ppm}$. 
(AA)-c, DMSO-d6, 500MHz, 298K

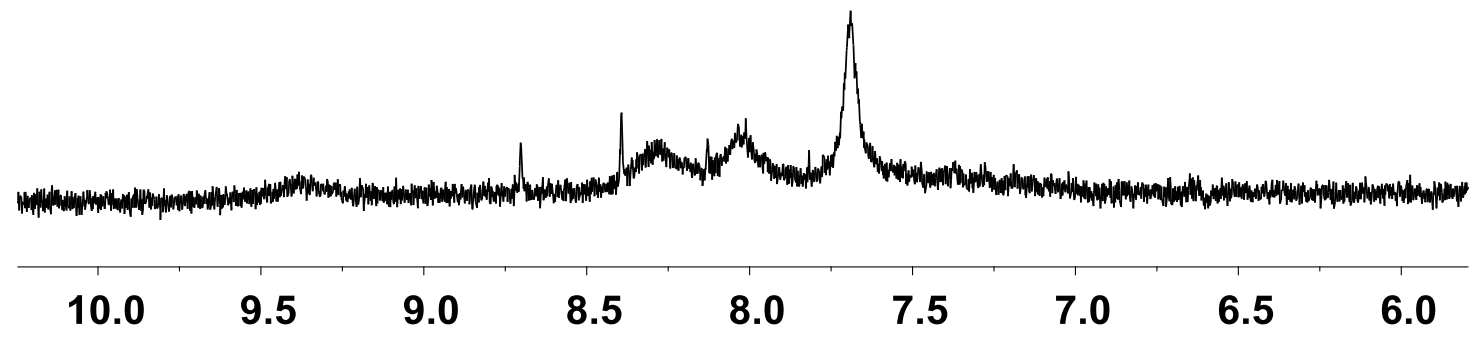

$\left(\right.$ AA) ${ }^{2}-\mathbf{c}$, DMSO-d6, 500MHz, 363K

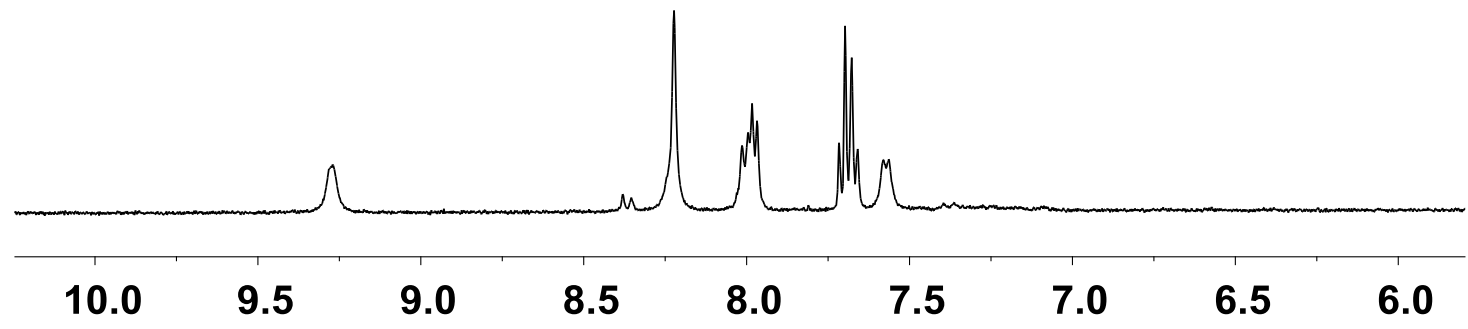

Figure S51. ${ }^{1} \mathrm{H}-\mathrm{NMR}$ variation with temperature, $298 \mathrm{~K}$ (top) and $363 \mathrm{~K}$ (down), of (AA) $)_{2}$-c in DMSO-d $\mathrm{d}_{6}$.

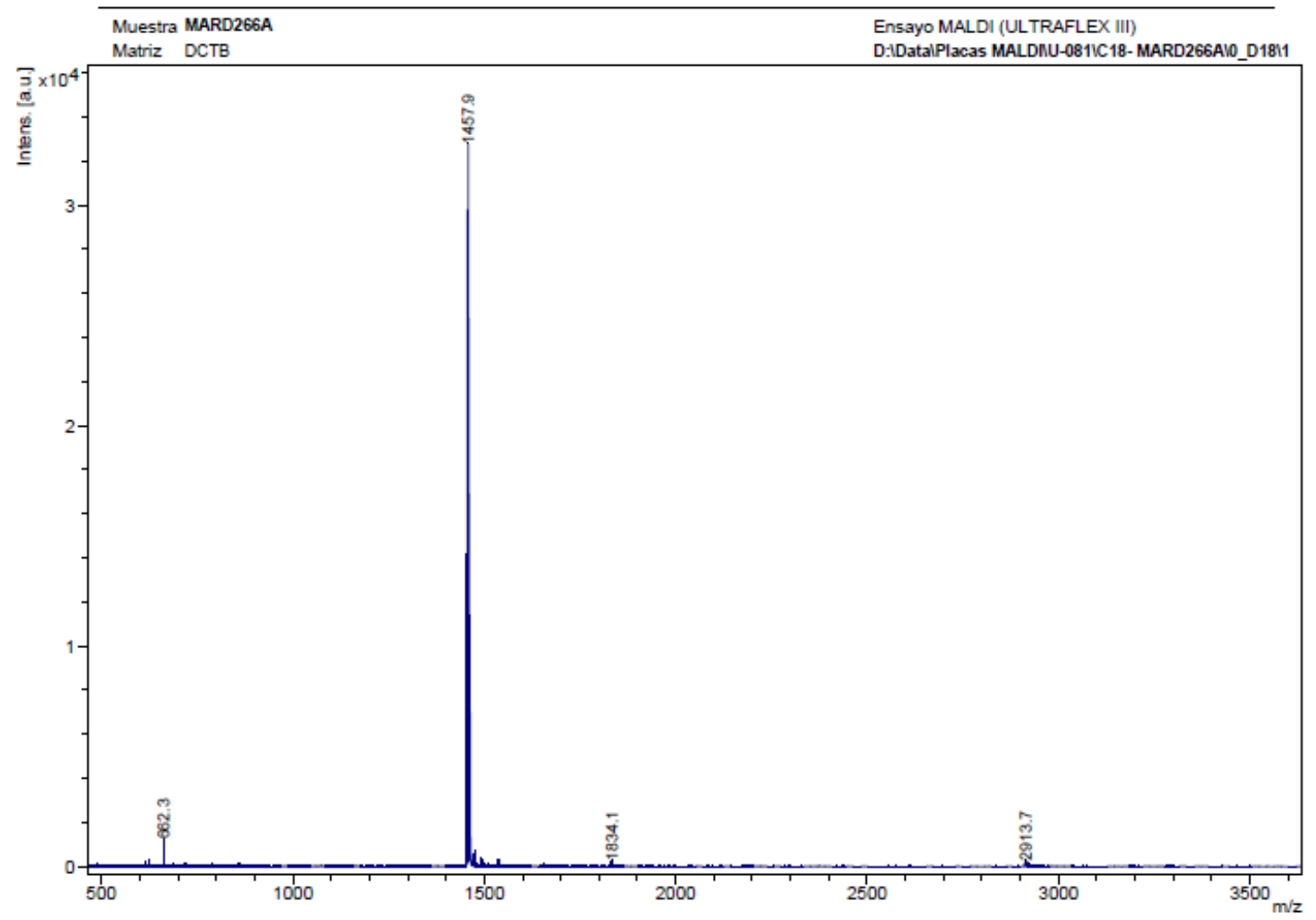




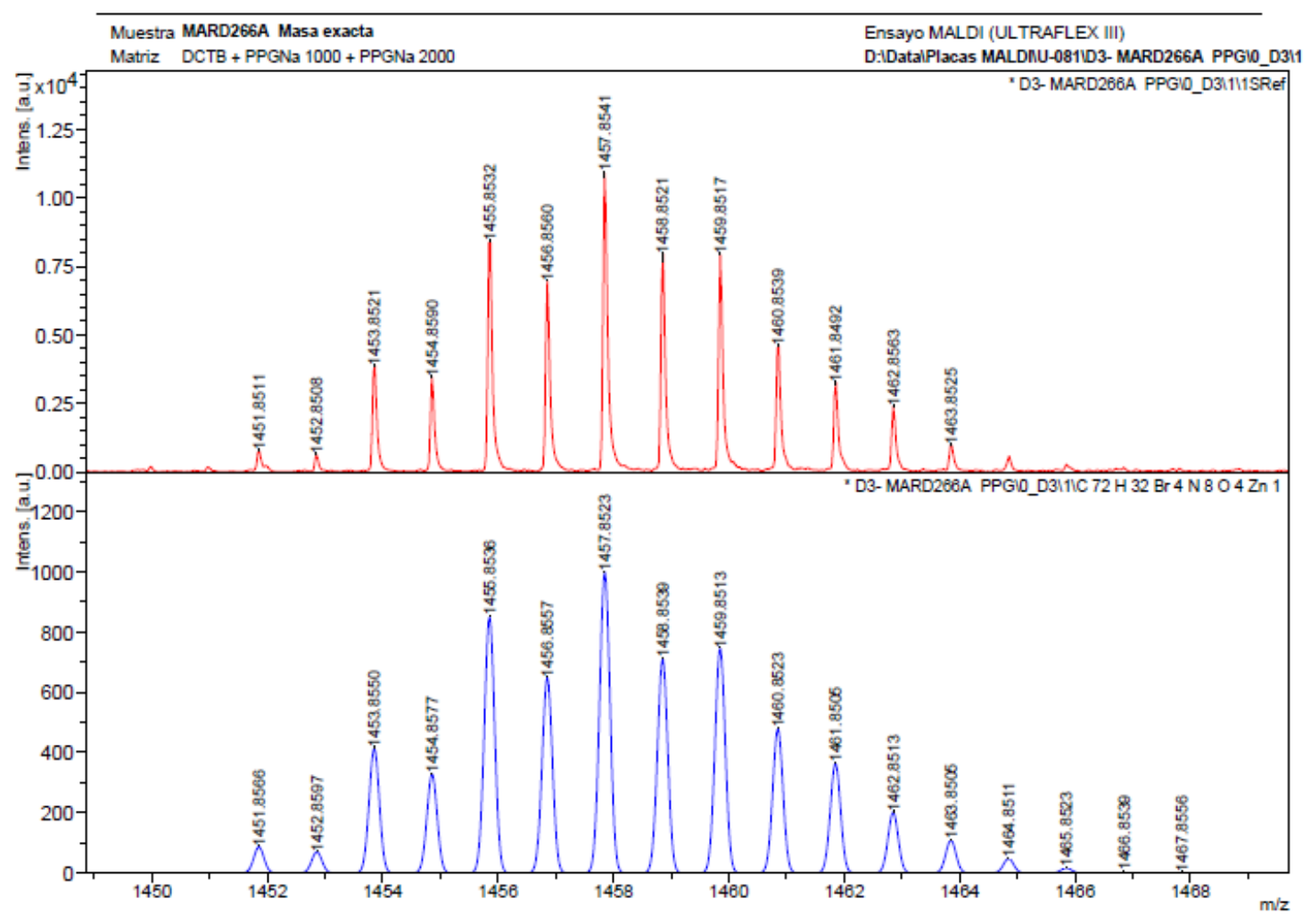

Figure S52. MS and HR-MS of $(A A)_{2}$-C.

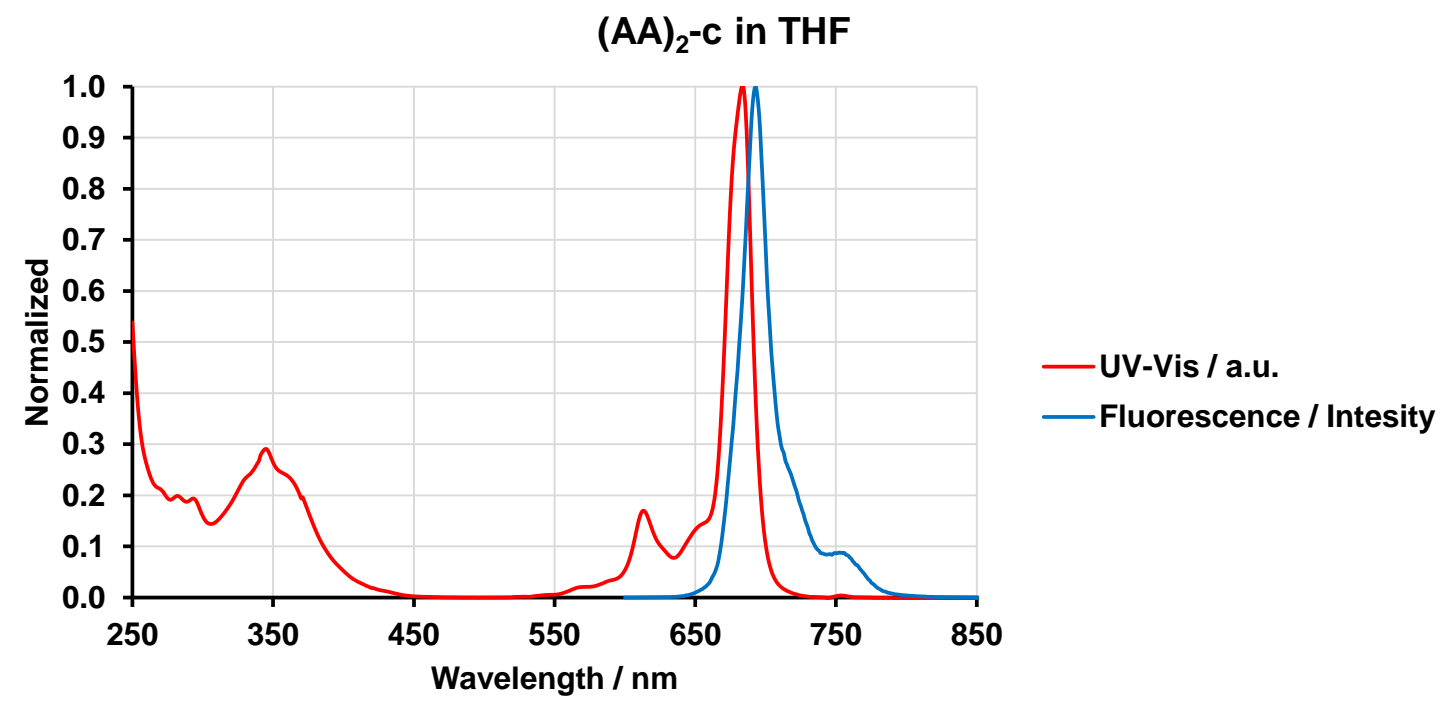

Figure S53. Normalized UV-Vis and Fluorescence spectra for (AA) $)_{2}-\mathrm{C}$ in THF. 


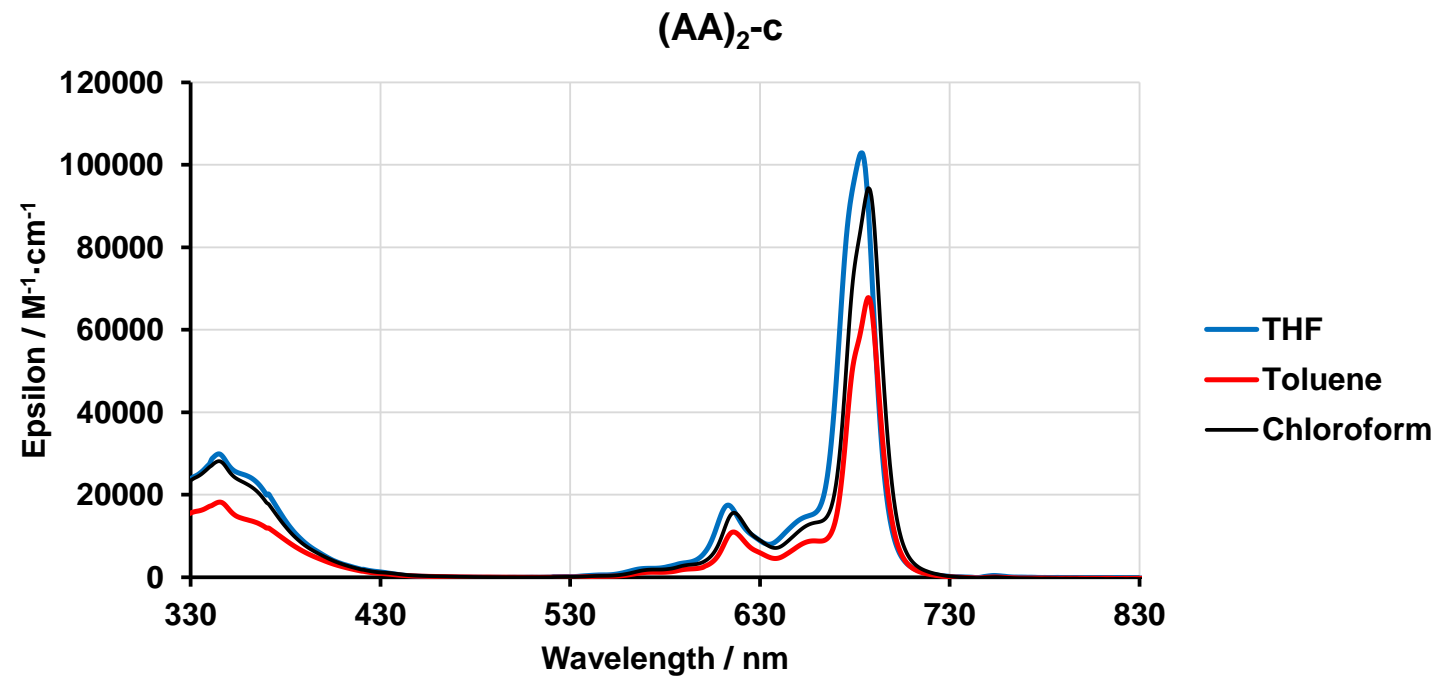

Figure S54. Normalized UV-Vis spectrum of (AA) $)_{2}-\mathrm{C}$ in THF, and toluene and $\mathrm{CHCl}_{3}$ as noncoordinative solvents. Aggregation phenomena are only clearly observed.

\section{$(\mathrm{R})-(\mathrm{AA})_{2}-\mathrm{C}$}

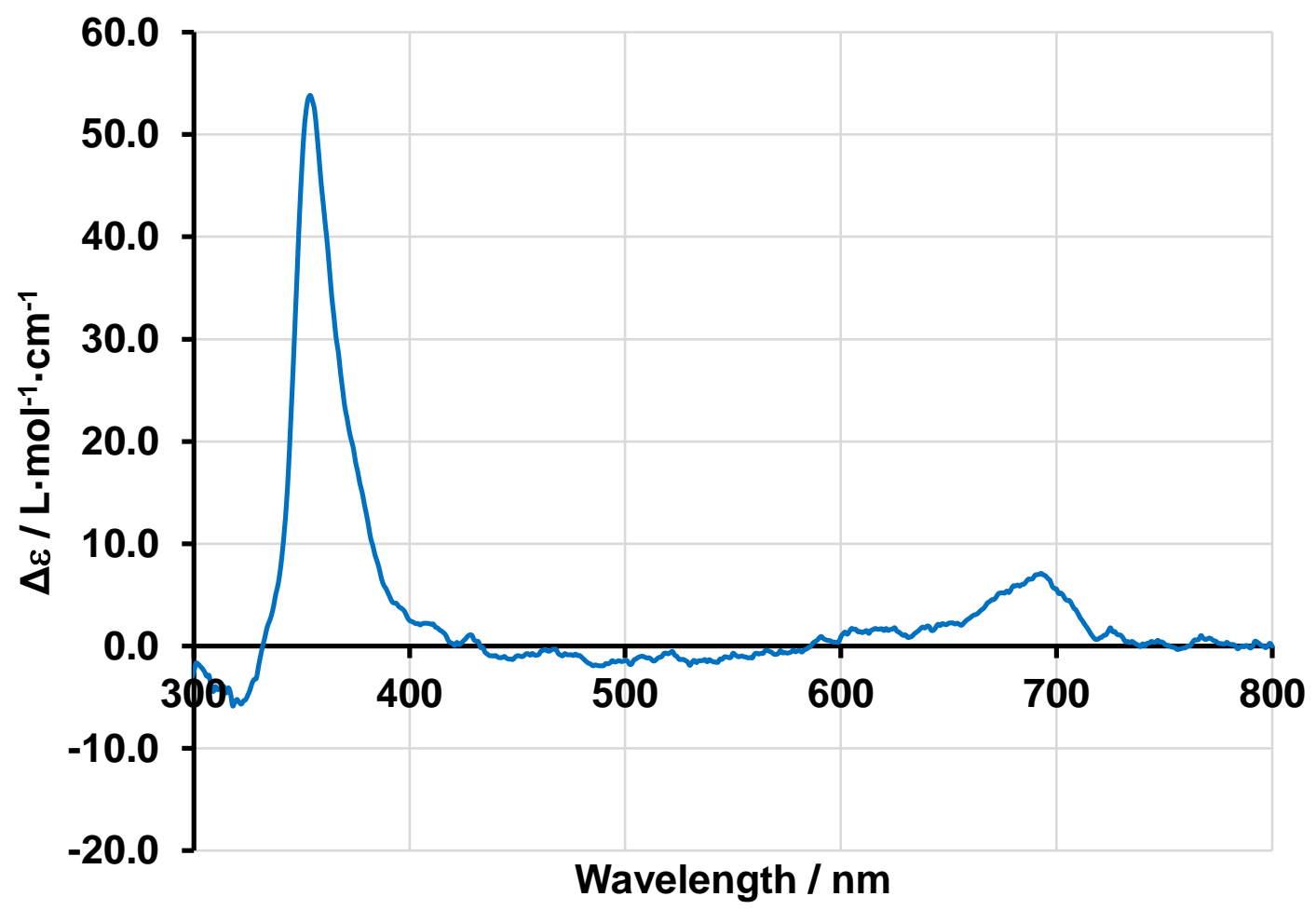

Figure S55. Normalized CD of $(A A)_{2}-\mathrm{C}$ in THF. 


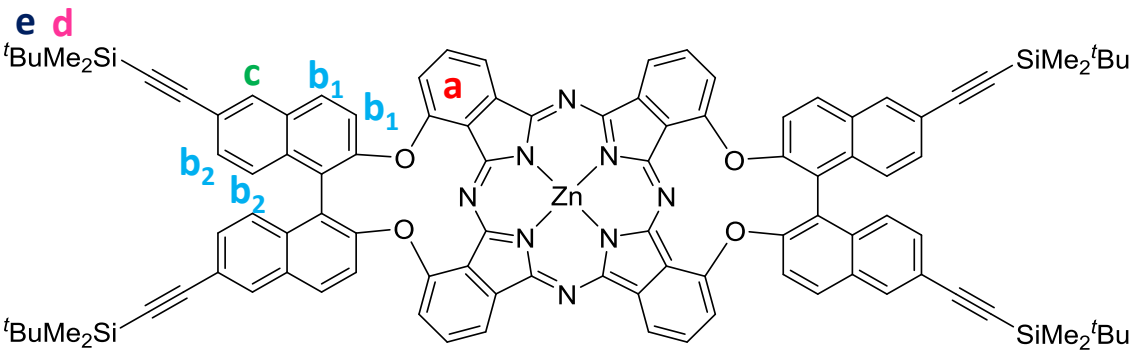

Figure S56. Structure of (AA) $)_{2}-d$ and $\mathrm{H}$ assignation for ${ }^{1} \mathrm{H}-\mathrm{NMR}$.

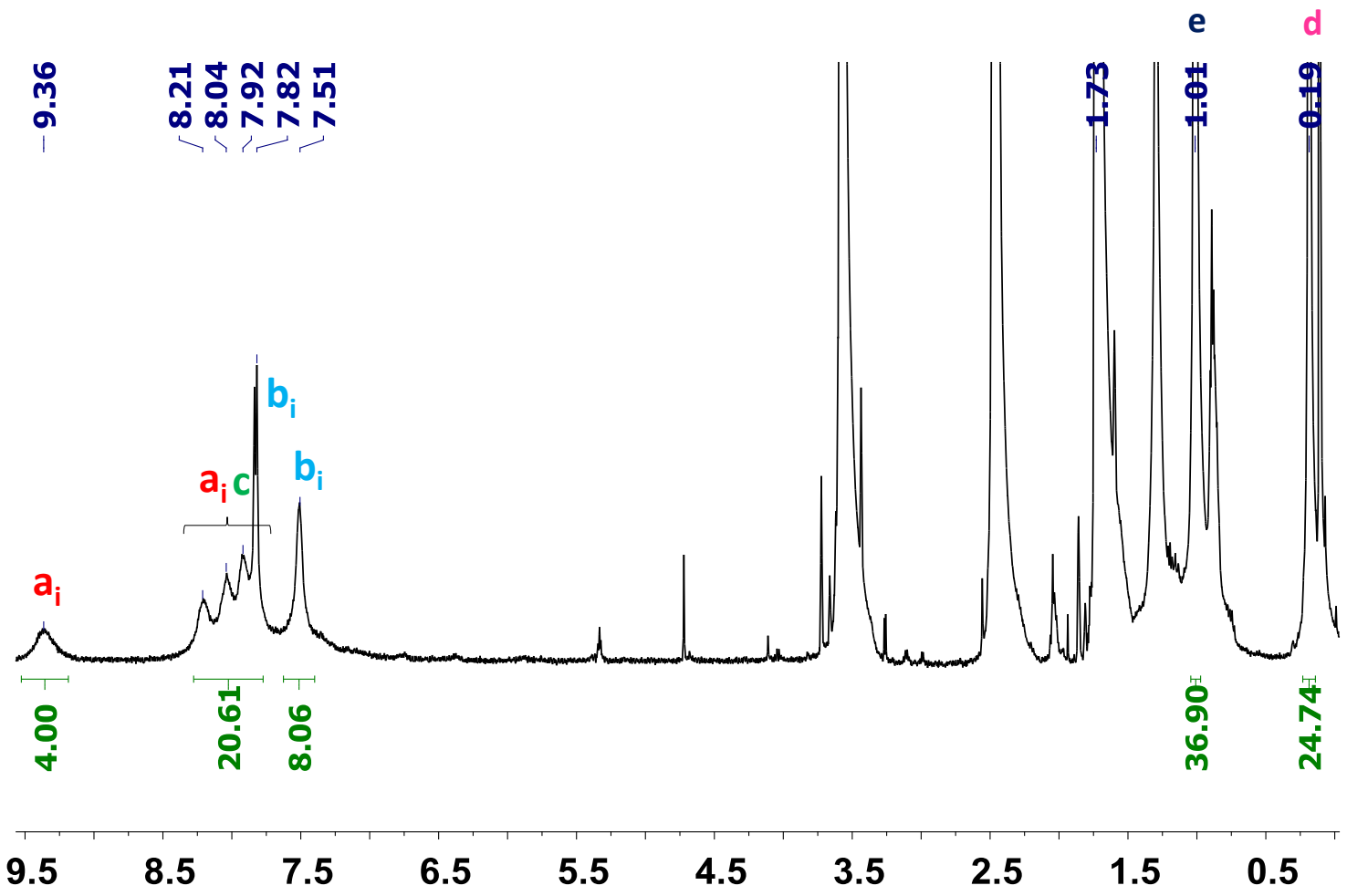




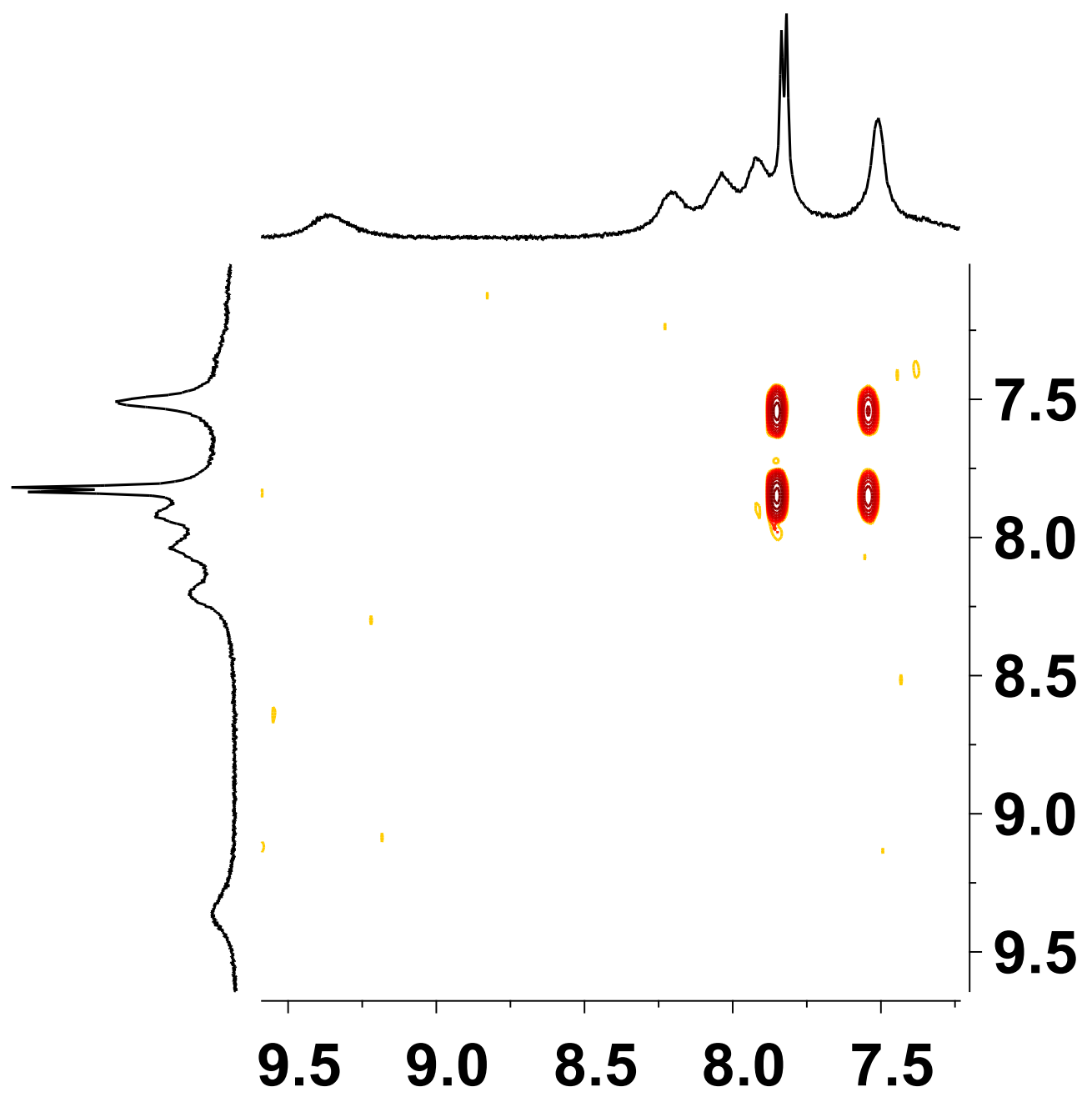

Figure S57. ${ }^{1} \mathrm{H}-\mathrm{NMR}$ and COSY of $(\mathrm{AA})_{2}-\mathrm{d}$ in THF- $\mathrm{d}_{8}$, at $298 \mathrm{~K}$. 
$(A A)^{2}-d$, THF-d8, 500MHz, 298K

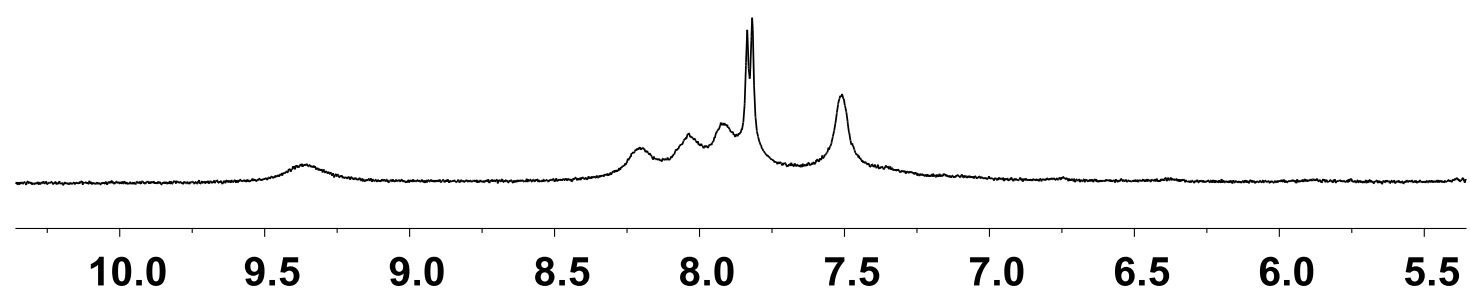

$(\text { AA) })^{2}-\mathrm{d}, \mathrm{THF}-\mathrm{d}^{8}, 500 \mathrm{MHz}, 323 \mathrm{~K}$

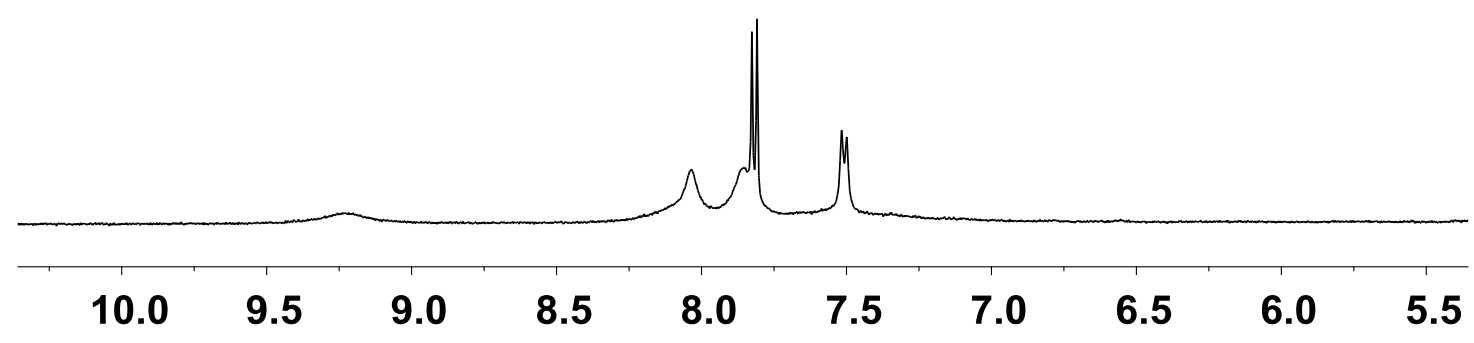

Figure S58. ${ }^{1} \mathrm{H}-\mathrm{NMR}$ variation with temperature, $298 \mathrm{~K}$ (top) and $323 \mathrm{~K}$ (down), of (AA) 2 -d in THF- $\mathrm{d}_{8}$.

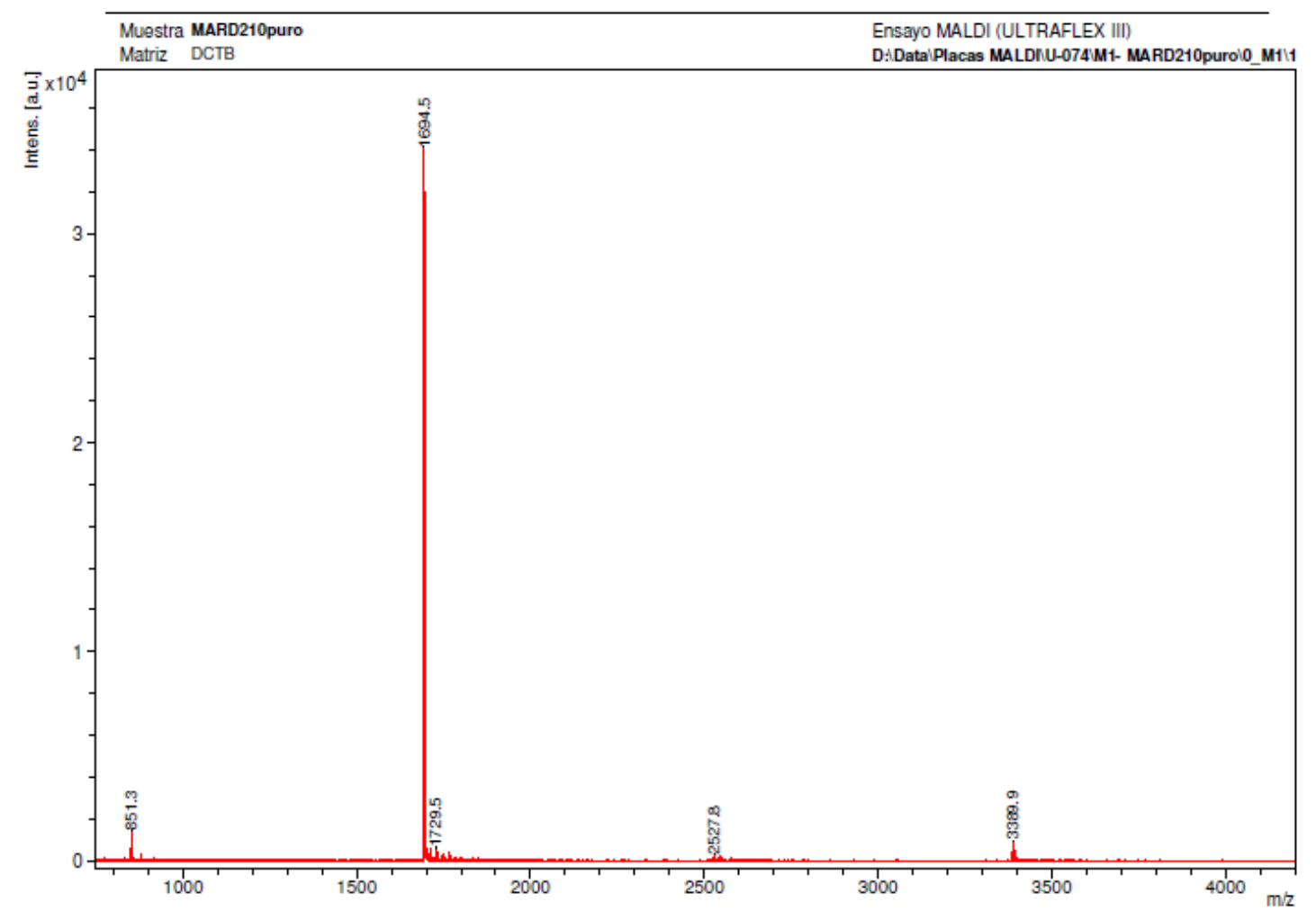




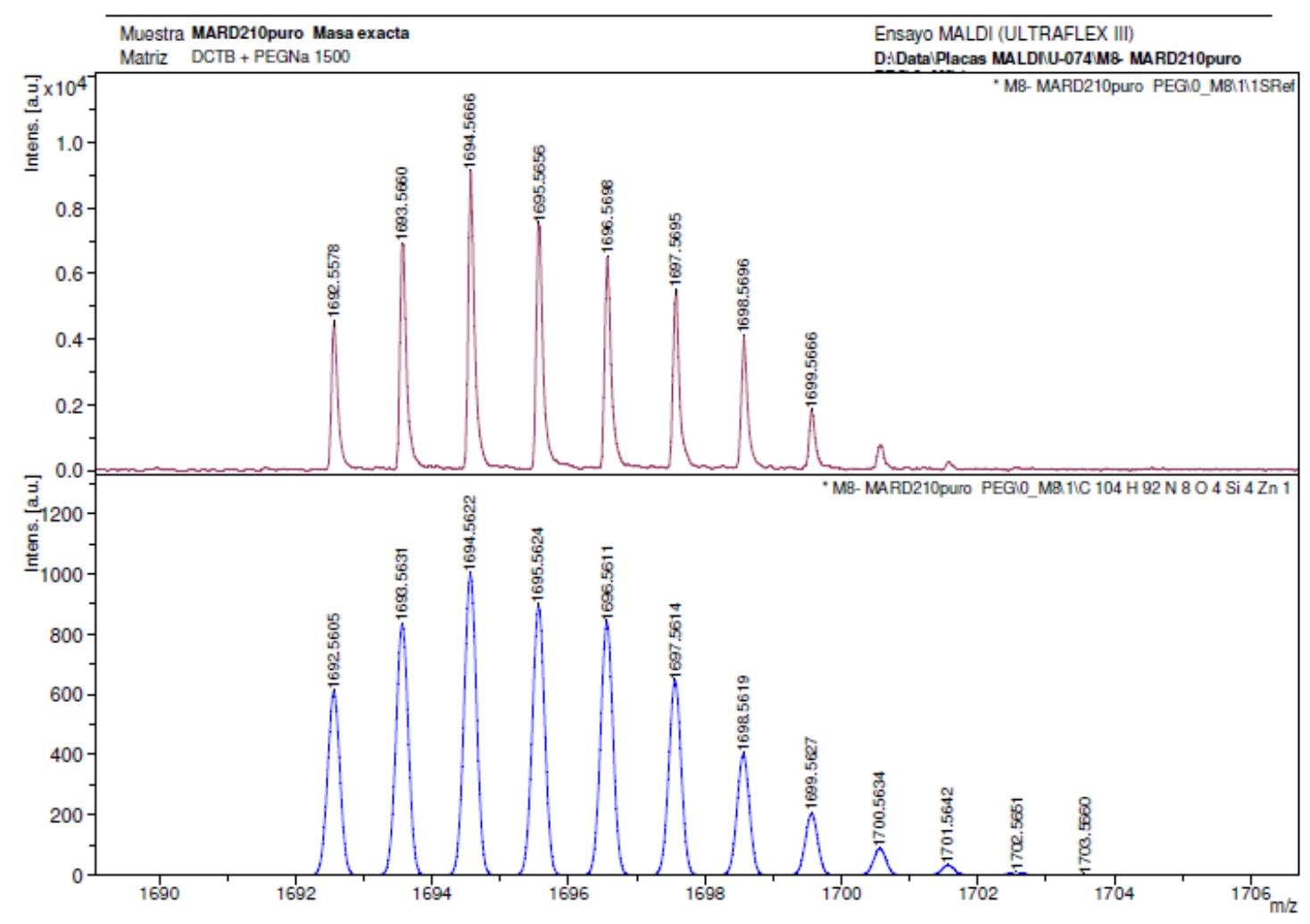

Figure S59. MS and HR-MS of (AA $)_{2}-d$.

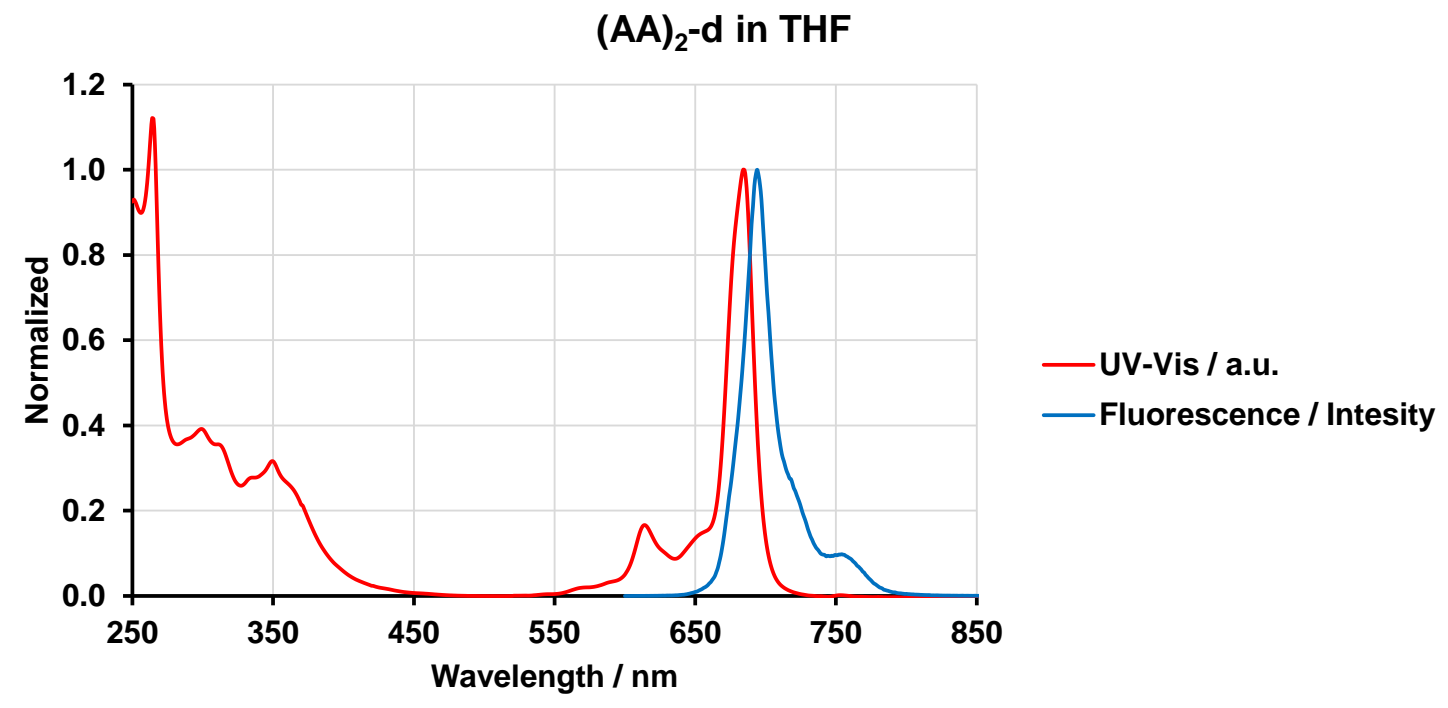

Figure S60. Normalized UV-Vis and Fluorescence spectra for $(A A)_{2}-\mathbf{d}$ in THF. 


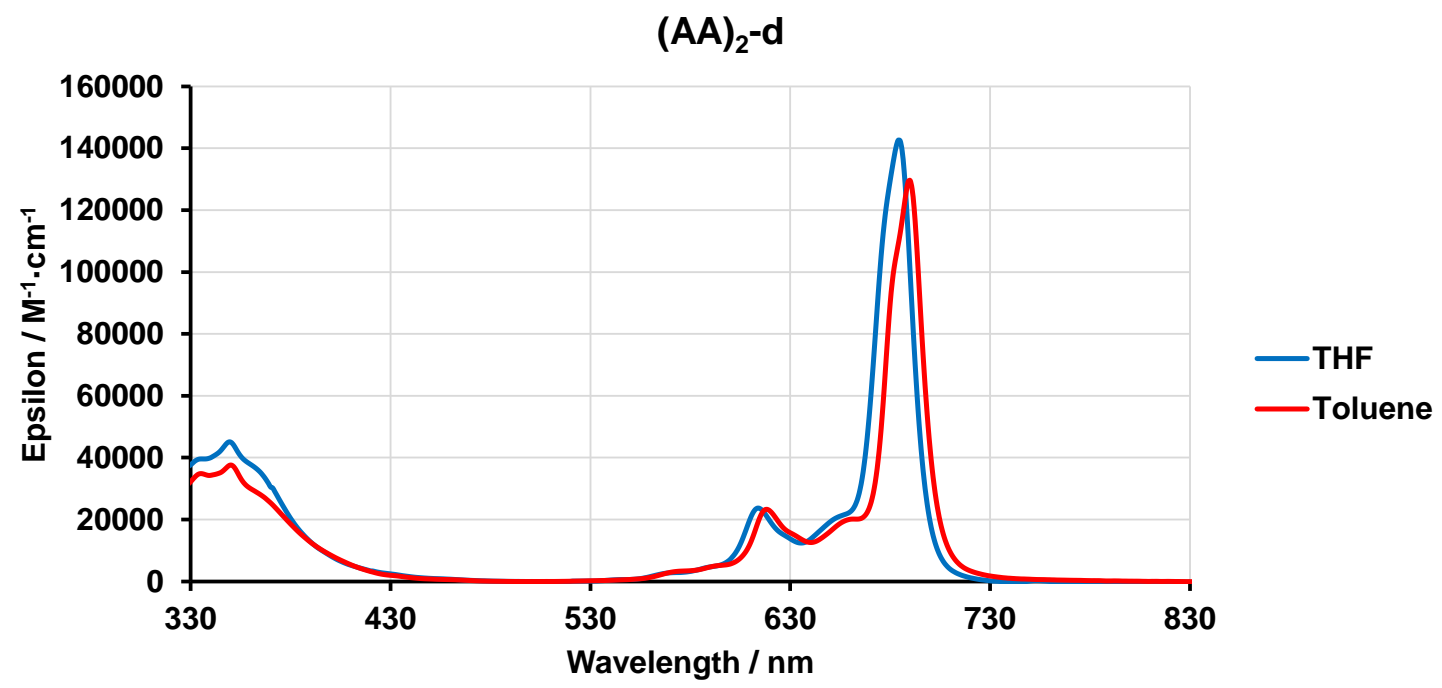

Figure S61. Normalized UV-Vis spectrum of (AA) $)_{2}-\mathrm{d}$ in THF, and toluene as non-coordinative solvent. Aggregation phenomena are not clearly observed.

$(R)-(A A)_{2}-d$

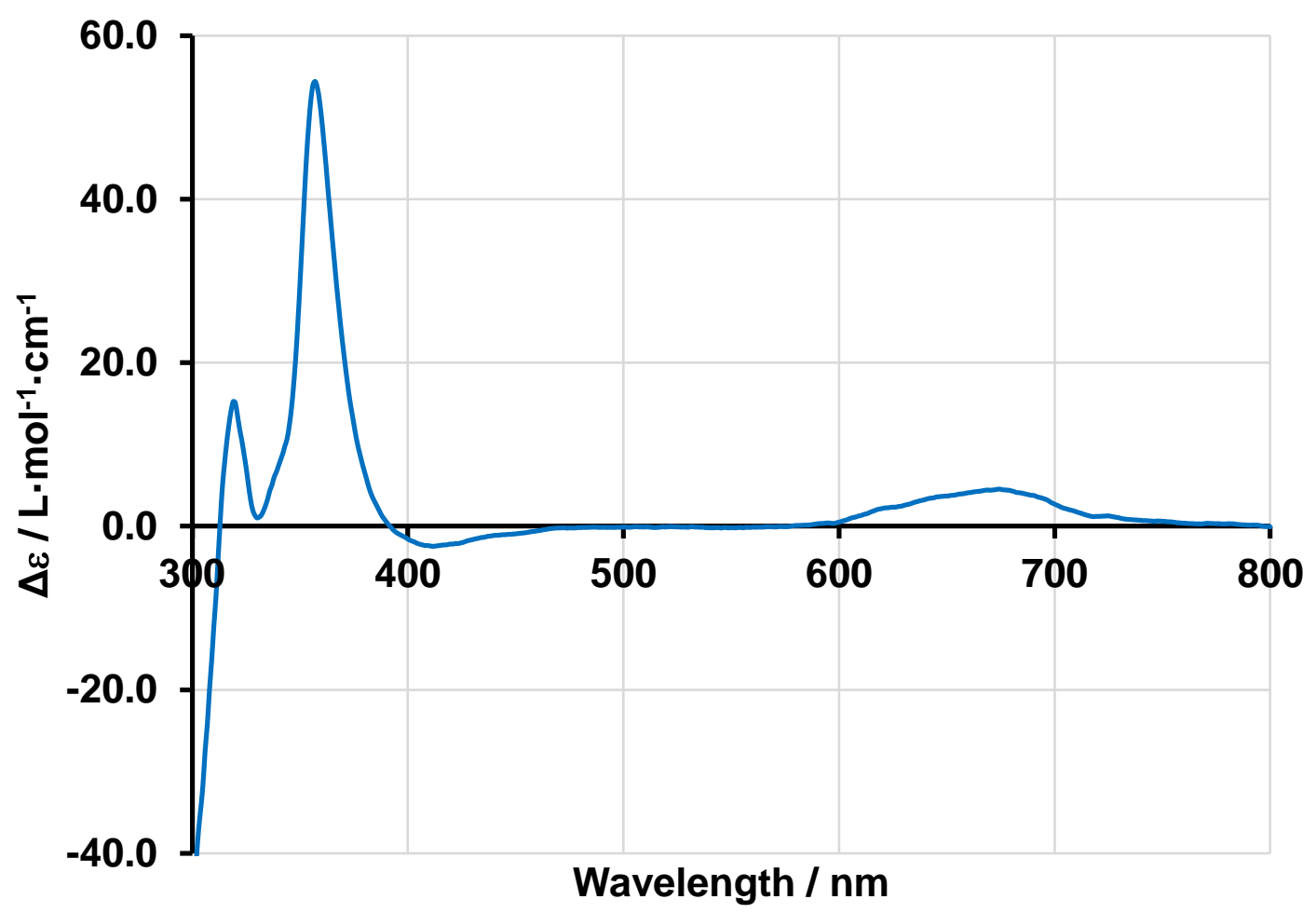

Figure S62. Normalized CD of (AA) ${ }_{2}$-d in THF. 